\title{
Paper \#4
}

\section{Auctions with Limited Commitment*}

\author{
Qingmin Liu ${ }^{\dagger}$ Konrad Mierendorff; and Xianwen $\mathrm{Shi}^{\S}$
}

December 14, 2013

\begin{abstract}
We study auction design in the standard symmetric independent private values environment, where the seller lacks the commitment power to withhold an unsold object off the market. The seller has a single object and can conduct an infinite sequence of standard auctions with reserve prices to maximize her expected profit. In each period, the seller can commit to a reserve price for the current period but cannot commit to future reserve prices. We analyze the problem with limited commitment through an auxiliary mechanism design problem with full commitment, in which an additional constraint reflects the sequential rationality of the seller. We characterize the maximal profit achievable in any perfect Bayesian equilibrium in the limit as the period length vanishes. The static full commitment profit is not achievable but the seller can always guarantee the profit of an efficient auction. If the number of buyers exceeds a cutoff which is small for many distributions, the efficient auction is optimal. Otherwise, the efficient auction is not optimal, and we give conditions under which the optimal solution consists of an initial auction with a non-trivial reserve price followed by a continuously decreasing price path. The solution is described by a simple ordinary differential equation. Our analysis combines insights from bargaining, auctions, and mechanism design.
\end{abstract}

\footnotetext{
${ }^{*}$ We wish to thank Jeremy Bulow, Yeon-Koo Che, Jacob Goeree, Philippe Jehiel, Navin Kartik, Alessandro Lizzeri, Steven Matthews, Benny Moldovanu, Bernard Salanié, Yuliy Sannikov, Vasiliki Skreta, Andrzej Skrzypacz, Philipp Strack, Alexander Wolitzky, and various seminar and conference audiences for helpful discussions and comments. Parts of this paper were written while some of the authors were visiting the University of Bonn, Columbia University, ESSET at the Study Center Gerzensee, Princeton University, and the University of Zurich. We are grateful for the hospitality of the respective institutions. Mierendorff gratefully acknowledges financial support from the Swiss National Science Foundation and the European Research Council (ESEI-249433). Shi gratefully acknowledges financial support by the Social Sciences and Humanities Research Council of Canada.

${ }^{\dagger}$ Columbia University, qingmin.liu@columbia.edu

${ }^{\ddagger}$ Columbia University and ESEI-University of Zurich, km2492@columbia.edu

${ }^{\S}$ University of Toronto, xianwen.shi@utoronto.ca
} 


\section{Contents}

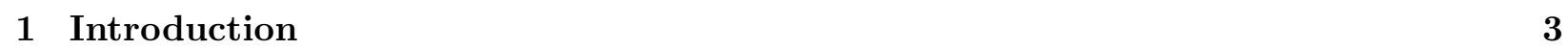

\begin{tabular}{|lrr}
\hline 2 & Model & 8
\end{tabular}

3 A Mechanism Design Approach to Limited Commitment 11

3.1 Direct Mechanisms . . . . . . . . . . . . . . . . . . . . . . . . . . . . . . . 12

3.2 Buyer Incentive Compatibility $\ldots \ldots \ldots \ldots \ldots$

3.3 The Payoff Floor Constraint $\ldots \ldots \ldots \ldots$

3.4 The Auxiliary Problem . . . . . . . . . . . . . . . . . . . . . . . . . 17

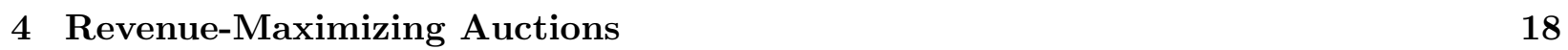

5 Connecting the Mechanism Design Approach to Discrete Time Equilibria 23

5.1 Discrete Time Approximation of the Efficient Auction . . . . . . . . . . . . . . . . 24

5.2 Discrete Time Approximation of the Solution to the Binding Payoff Floor Constraint 25

5.3 Optimality of the Solution to the Auxiliary Problem . . . . . . . . . . . . . . 27

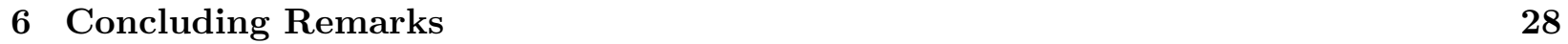

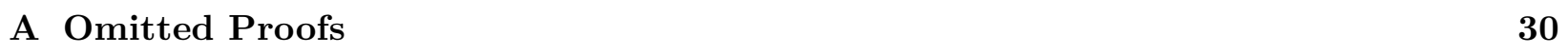

A.1 Proofs for Section $2 \ldots \ldots \ldots \ldots \ldots \ldots$

A.2 Proofs for Section $3 \ldots \ldots \ldots \ldots \ldots \ldots \ldots$

A.3 Proofs for Section $4 \ldots \ldots \ldots \ldots$

A.3.1 Step 1: Construct a Solution to the Binding Payoff Floor Constraint . . . . . 42

A.3.2 Step 2: Check Feasibility of the Constructed Solution . . . . . . . . . . . . . 50

A.3.3 Step 3: Prove Optimality of the Constructed Solution . . . . . . . . . . . . . 57

A.4 Proofs for Section $51 \ldots \ldots \ldots \ldots \ldots$

A.4.1 Construction of a Solution to the Generalized Payoff Floor Constraint . . . . 66

A.4.2 Discretization . . . . . . . . . . . . . . . . 75

B Existence of Weak Markov Equilibria and the Uniform Coase Conjecture 81

B.1 Proof of Proposition $5 \ldots \ldots \ldots \ldots \ldots \ldots$

B.2 Proof of Proposition $6 \ldots \ldots \ldots \ldots \ldots \ldots \ldots \ldots$ 


\section{Introduction}

Auction theory has found many applications ranging from private and public procurement to takeover bidding and electronic commerce. Auctions are also gaining popularity in many newly set-up markets for allocating resources such as energy, transportation, radio spectrum, and pollution permits. Conceptually, auction design is the focal application of the mechanism design theory, which is now a standard tool for economic analysis. The vast majority of prior work on revenue maximizing auctions has as its starting point the celebrated work of Myerson (1981) and Riley and Samuelson (1981). Under a regularity condition, the optimal auction format is a standard auction (e.g., a second-price auction or a first-price auction) with a reserve price strictly above the seller's reservation value. Consequently, a revenue-maximizing auction prescribes an inefficient exclusion of some low-valued buyers.

To implement the optimal auction, it is crucial for the seller to be able to commit to withholding an unsold object off the market permanently. If no bidders bid above the predetermined reserve price, the seller has to stop auctioning the object even though there is common knowledge of unrealized gains from trade. From a theoretical perspective, the full commitment assumption is crucial for the validity of the revelation principle - an important tool that greatly simplifies the analysis and has been instrumental for the advancement of the theory of auctions and more broadly mechanism design. This assumption, however, is not entirely satisfactory in many applications. For example, in the sale of art and antiques, real estate, automobiles, and spectrum licenses, aborted auctions are common, but the destruction of unsold objects is rare or infeasible. Unsold objects are often re-auctioned or offered for sale later 1 As such, understanding the role of commitment in an auction setting is of both theoretical and practical importance. In this paper, we take a step in this direction.

We revisit the classic auction model with one seller, a single indivisible object, and multiple buyers, whose values are drawn independently from a common distribution. Different from the classic auction model, if the object is not sold on previous occasions, the seller can sell it again with no predetermined deadline. At the same time, we restrict attention to standard auction formats with reserve prices rather than general mechanisms. More precisely, in each time period until the object is sold, the seller posts a reserve price and holds a second-price auction 2 Each buyer can either wait for a future auction or submit a bid no smaller than the reserve price. Waiting is costly and both the buyers and the seller discount at the same rate. Within a period, the seller is

\footnotetext{
${ }^{1}$ For example, in the $2 \mathrm{G}$ spectrum auction run by the Indian government in November 2012, nearly half of the spectrum blocks put up for sale went unsold due to high reserve prices. When asked about future government plans, Kapil Sibal, the minister of communications and information technology, said, "Of course there will be an auction. There is no doubt about that." (http://newindianexpress.com/business/news/article1340726.ece). Failed auctions are also common in U.S. government sale for offshore oil and gas leases (Porter 1995), and in markets for fine arts where around $20-30 \%$ of the objects up for sale do not sell at auction as reported by the Wall Street Journal (April 23, 2008).

${ }^{2}$ Allowing the seller to choose between standard auctions will not change our analysis and results.
} 
committed to the rules of the auction and the announced reserve price. The seller cannot, however, commit to future reserve prices.

This simple setting is sufficiently rich to investigate the role of commitment. In our model, the seller's commitment power varies with the period length (or effectively with the discount factor). If the period length is infinite, then the seller has full commitment power. As the period length shrinks, the seller's commitment power also diminishes. We adopt the solution concept of perfect Bayesian equilibrium, which is well-defined for the discrete-time game, and develop a method to analyze the continuous-time limit at which the seller's commitment power vanishes. Within this framework, we address the following questions: what is the set of equilibrium payoffs that is attainable by the seller, and what is the equilibrium selling strategy that attains the maximal payoff? We find that the full commitment profit is no longer achievable, and moreover, an efficient auction can be revenue-maximizing. These results provide a deeper understanding of the role of commitment power in auctions.

To illustrate, assume that the buyers' valuations are uniformly distributed on $[0,1]$, and the seller has a reservation value of 0 . If there are two buyers, then in the continuous time limit, the revenue-maximizing equilibrium involves an initial auction with reserve price $\frac{4}{9}$, followed by a reserve price path given by $p_{t}=\frac{4}{9} e^{-r t}$, where $r$ is the common discount rate. The resulting revenue for the seller is $\frac{31}{81}$, which is strictly below the revenue from Myerson's optimal auction $\left(\frac{5}{12}\right)$, but is strictly higher than the revenue from an efficient auction $\left(\frac{1}{3}\right)$. Relative to the efficient auction benchmark, the seller achieves more than half of the additional profit that can be extracted with full commitment power. In contrast, if there are three or more buyers, an efficient auction-which is given by $p_{t}=0$ for all $t$-is revenue-maximizing in the continuous time limit.

We are not the first to investigate the commitment assumption in auctions. A natural departure from full commitment is to consider a model with a finite number of periods where the seller can propose and commit to a mechanism in each period but cannot commit to mechanisms for future periods. This is the approach taken by Skreta (2006, 2011). In her model, the seller effectively has full commitment power in the last period and the backward induction logic applies. In fact, the seller has the option to wait until the last period to run a Myerson optimal auction, which attains the full commitment profit if she is patient. Despite the complications that arise with a general class of mechanisms under limited commitment, Skreta shows that the optimal mechanism is a sequence of standard auctions with reserve prices $3^{3}$ The assumption of a finite horizon fits important applications, such as flight or concert ticket sales, where the good must be consumed before a fixed date. With durable goods and no binding deadline for a transaction, a finite horizon model cannot fully capture the spirit of non-commitment. In line with the literature on dynamic

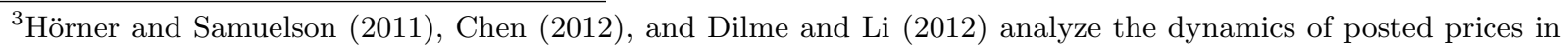
a finite horizon model. They assume that the winner is selected randomly when multiple buyers accept the posted price.
} 
games, we find that our infinite horizon model generates equilibrium predictions which are very different from the finite horizon model.

Several conceptual issues arise in the analysis of the seller's maximal payoff in our model. First, the full commitment solution is not feasible. Therefore, we have to characterize the set of feasible solutions first. Second, with an infinite horizon, we cannot rely on backward induction to identify an equilibrium. Finally, like Skreta, we cannot rely on the revelation principle because the seller has limited commitment 4

The key idea we employ is to translate the limited-commitment problem into an auxiliary mechanism design problem with full commitment. In the auxiliary problem, we impose an additional restriction on the feasible solutions. The restriction reflects a necessary sequential rationality constraint faced by the seller in the original limited-commitment problem. Formally, the seller maximizes revenue by choosing and committing to an auxiliary direct mechanism, which specifies the trading time and transfers for every profile of reported buyer types in continuous time. If there are no additional restrictions other than incentive compatibility and participation constraints, the optimal solution is simply the optimal auction with full commitment: a buyer trades at time 0 if his valuation is the highest and exceeds some predetermined reserve price, while all other buyers never trade. In particular, if the valuation of the highest buyer is below the reserve price, no trade will occur. The seller keeps the object and expects to earn zero continuation profit at every point in time. This is in contrast to our original problem where the seller's continuation value is bounded away from zero - as we show, she can always run an efficient auction to end the game at any point in time. Therefore, we impose a "payoff floor" constraint in the auxiliary problem as a necessary condition for sequential rationality: at any point in time, the seller's continuation payoff in the auxiliary mechanism must be bounded below by the payoff from an efficient auction for the corresponding posterior belief 5 The value of the auxiliary problem provides an upper bound for the equilibrium payoffs in the original game (in the continuous-time limit). We proceed to solve the auxiliary problem and show that its value and its solution can be approximated by a sequence of equilibrium outcomes of the original game. Therefore, the value of the auxiliary problem is precisely the maximal attainable equilibrium payoff in our original problem, and the solution to the auxiliary problem is precisely the limiting selling strategy that attains this maximal payoff: 6

Using the auxiliary problem, we obtain the following results. First, the full commitment profit cannot be achieved under limited commitment because the reserve price must converge to zero

\footnotetext{
${ }_{4}^{\text {Bester and Strausz }} \sqrt{2001)}$ develop a version of the revelation principle with limited commitment for environments with one agent and a finite number of periods. It does not apply to our setting because our model has multiple buyers (Bester and Strausz, 2000).

${ }^{5}$ Notice that, for a given auxiliary mechanism, the seller knows exactly which set of types are left at each moment in time, if the mechanism is carried out. Consequently, she can compute the posterior beliefs as well as her continuation payoff from the given mechanism.

6 Wolitzky (2010) uses an auxiliary problem to analyze a Coasian bargaining model in which the seller cannot commit to delivery. His auxiliary problem is much simpler because in his model there is always a no-trade equilibrium, which allows him to show that the seller can achieve the full commitment profit.
} 
for any feasible solution. Second, the efficient auction is optimal if the number of bidders exceeds a cutoff which depends on the type distribution 7 In this case, the efficient auction is also the only feasible solution to the auxiliary problem. Third, if the number of bidders falls short of the aforementioned cutoff, strictly positive reserve prices are feasible and the efficient auction is not optimal. The cutoff only depends on the lower tail of the type distribution, because positive reserve prices have to be sustainable in continuation games where the posterior type distributions are the lower tail truncations of the prior type distribution. Finally, under the assumption that the monopoly profit function is concave, the payoff floor constraint binds everywhere at the optimal solution. This yields an ordinary differential equation that describes the optimal solution if the efficient auction is not optimal. In this solution, the seller uses an auction with a strictly positive reserve price at time zero. After the initial auction, the seller uses a smoothly declining price path which is strictly positive and converges to zero only at infinity.

An alternative approach modeling limited commitment is to assume that the seller cannot commit to trading rules even for the present period. This is the approach taken by McAdams and Schwarz (2007) and Vartiainen (2013). McAdams and Schwarz (2007) consider an extensive form game in which the seller can solicit multiple rounds of offers from buyers. Their paper shows that if the cost of soliciting another round of offers is large, the seller can credibly commit to a first-price auction, and if the cost is small, the equilibrium outcome approximates that of an English auction. In Vartiainen (2013), a mechanism is a pure communication device that permits the seller to receive messages from bidders. The seller cannot commit to any action after receiving the messages, and there is no discounting. Vartiainen shows that the only credible mechanism is an English auction because it reveals just the right amount of information such that it is optimal for the seller to respect the rules of the auction. In contrast to these papers, we posit that the seller cannot renege on the agreed terms of the trade. For example, this might be enforced by the legal environment.

A special case of our setup is the model of bilateral bargaining in which an uninformed seller makes price offers to a single privately informed buyer. This model is equivalent to a durable goods monopoly with a continuum of buyers. In his seminal paper, Coase (1972) argues that a price-setting monopolist completely loses his monopoly power if she has the option to revise prices frequently. Game theoretic analysis shows that there are in fact two types of equilibria in the Coasian bargaining model (see Fudenberg, Levine, and Tirole, 1985, Gul, Sonnenschein, and Wilson, 1986, Ausubel and Deneckere, 1989) 8 In the "gap" case, where the seller's reservation value is strictly below the lowest valuation of the buyer, every equilibrium satisfies the Coase conjecture.

\footnotetext{
${ }^{7}$ In the case of the uniform distribution, the cutoff is between two and three as illustrated above. This cutoff applies more generally to all distributions with a strictly positive density that has a bounded derivative.

${ }^{8}$ See also Stokey (1981) and Bulow (1982). The commitment issue in durable goods monopoly has been analyzed in richer environments, see, e.g. Sobel (1991) and Fuchs and Skrzypacz (2010) for models with entry of new buyers. Ausubel, Cramton, and Deneckere (2002) survey the extensive literature on bilateral bargaining and the Coase conjecture. Strulovici (2013) considers a more general setting in which the two parties can negotiate and renegotiate long-term contracts instead of just prices.
} 
In the limit, as the period length converges to zero, the prices posted by the seller converge to the lowest buyer-valuation and her profit converges to the competitive level. A Coase conjecture result for the "gap" case in an auction setting is obtained by McAfee and Vincent (1997).

Our model corresponds to the "no gap" case. The equilibrium construction, which we use to justify our auxiliary mechanism design approach, draws insights from Ausubel and Deneckere (1989). They show that there exist equilibria of the bilateral bargaining model in which the seller can credibly maintain high prices. Their equilibrium price path is sustained by a trigger strategy: as long as the seller plays "tough" by following a slowly decreasing price path, the buyer best responds to the equilibrium price path. If the seller deviates, the buyer switches to the Coasian equilibrium strategy, forcing the seller to lower prices quickly and to obtain a profit close to zero. Since the punishment profit converges to zero in the limit, the equilibrium price path can be calibrated to approximate the full commitment solution.

In our model with multiple buyers, this reasoning does not apply directly. In particular, we show that the seller can guarantee herself at least the revenue from an efficient auction which is strictly positive. This severely restricts the effectiveness of the punishment after a deviation, so that the full commitment revenue is not attainable. We give general conditions under which the punishment is completely futile even for a small numbers of bidders. When these conditions are not met, our analysis characterizes the maximal attainable revenue, and also confirms the intuition that an efficient auction is indeed the most effective punishment and a trigger strategy equilibrium maximizes the seller's payoff.

There is also a recent and growing literature on dynamic mechanism design. See for example Courty and Li (2000), Pavan, Segal, and Toikka (2013), Board and Skrzypacz (2013) and Eso and Szentes (2007) among many others, as well as the surveys by Bergemann and Said (2011) and Gershkov and Moldovanu (2012). This literature focuses on models with dynamic populations, dynamic arrival of information, and learning, and often assumes full commitment power. We did not consider such applications in order to focus on the central issue of limited commitment.

The paper is organized as follows. In the next section, we formally introduce the model and develop some structural properties of equilibria in discrete time. In Section 3 , we introduce the auxiliary full commitment mechanism design problem. Section 4 contains our main results regarding optimal solutions to the auxiliary problem. Section 5 links the mechanism design problem with full commitment to the original problem of finding seller optimal equilibria in the discrete time game. Section 6 concludes with a discussion of our main assumptions and directions for future research. All proofs are contained in the Appendix. 


\section{Model}

Consider the standard auction environment where a seller (she) wants to sell an indivisible object to $n$ potential buyers (he). Buyer $i$ privately observes his own valuation for the object $v^{i} \in[0,1]$. We use $\left(v^{i}, v^{-i}\right) \in[0,1]^{n}$ to denote the vector of the $n$ buyers' valuations, and $v \in[0,1]$ to denote a generic buyer's valuation. Each $v^{i}$ is drawn independently from a common distribution $F(\cdot)$ with full support and continuously differentiable density $f(\cdot)$ such that $f(v)>0$ for all $v \in(0,1)$. The highest order statistic of the $n$ valuations $\left(v^{i}, v^{-i}\right)$ is denoted by $v^{(n)}$ and its corresponding distribution by $F^{(n)}$. The seller's reservation value for the object is 0.9

Time is discrete and the period length is denoted by $\Delta$. In each period $t=0, \Delta, 2 \Delta, \ldots$, the seller runs a second-price auction (SPA) to sell the object if it has not been sold yet ${ }^{10}$ To simplify notation, we often do not explicitly note the dependence of the game on $\Delta$. The timing within period $t$ is as follows. First, the seller publicly announces a reserve price $p_{t}$ for the auction run in period $t$ and invites all buyers to submit a valid bid, which is restricted to the interval $\left[p_{t}, 1\right]$. After observing $p_{t}$, all buyers decide simultaneously either to bid or to wait. If at least one valid bid is submitted, the winner and the payment is determined according to the rules of the second-price auction and the game ends. If no valid bid is submitted, the game proceeds to the next period. Both the seller and the buyers are risk-neutral and have a common discount rate $r>0$ (or a discount factor per period equal to $\delta=e^{-r \Delta}<1$ ). If buyer $i$ wins in period $t$ and has to make a payment $\pi^{i}$, then his payoff is $e^{-r t}\left(v^{i}-\pi^{i}\right)$ and the seller's payoff is $e^{-r t} \pi^{i}$.

We assume that the seller has limited commitment power. She can commit to the auction format and the reserve price that she announces for the current period: if a valid bid is placed, then the object is sold according to the proposed auction and she cannot renege. She cannot commit, however, to future reserve prices: if the object was not sold in a period, the seller can always run another auction with a new reserve price in the next period. She cannot promise to stop auctioning an unsold object, or commit to a predetermined sequence of reserve prices.

Denote by $h_{t}=\left(p_{0}, p_{\Delta}, \ldots, p_{t-\Delta}\right)$ the public history at the beginning of $t$ if no bidder has placed a valid bid up to $t$ and write $h_{0}=\varnothing$ for the history at which the seller chooses the first reserve price ${ }^{11}$ Let $H_{t}$ be the set of such histories. A (behavioral) strategy for the seller specifies a function $p_{t}: H_{t} \rightarrow \mathcal{P}[0,1]$ for each $t=0, \Delta, 2 \Delta, \ldots$, where $\mathcal{P}[0,1]$ is the space of Borel probability measures endowed with the weak* topology. A (behavioral) strategy for buyer $i$ specifies a function $b_{t}^{i}: H_{t} \times[0,1] \times[0,1] \rightarrow \mathcal{P}[0,1]$ for each $t=0, \Delta, 2 \Delta, \ldots$, where we assume that $b_{t}^{i}\left(h_{t}, p_{t}, v^{i}\right)$ is Borel-measurable in $v^{i}$, for all $h_{t} \in H_{t}$, and all $p_{t} \in[0,1]$, and that $\operatorname{supp} b_{t}^{i}\left(h_{t}, p_{t}, v^{i}\right) \subset\{0\} \cup\left[p_{t}, 1\right]$.

We consider perfect Bayesian equilibria (PBE), and we will focus on equilibria that are buyer

\footnotetext{
${ }^{9}$ The important assumption here is that the seller's reservation value is no smaller than lower bound of the support of the buyers' values, i.e., we are in the "no-gap" case. The rest is normalization.

${ }^{10}$ Our analysis does not change if the seller uses any standard auction instead.

${ }^{11}$ We do not have to consider other histories because the game ends if someone places a valid bid.
} 
symmetric ${ }^{12}$ We will not distinguish between strategies that coincide with probability one for all histories. In the rest of the paper, "equilibrium" is used to refer to this class of perfect Bayesian equilibria. Let $\mathcal{E}(\Delta)$ denote the set of equilibria of the game for given $\Delta$. Let $\Pi^{\Delta}(p, b)$ denote seller's expected revenue derived from equilibrium $(p, b) \in \mathcal{E}(\Delta)$. We are interested in the maximal profit the seller can achieve in the limit when the period length vanishes:

$$
\Pi^{*}:=\limsup _{\Delta \rightarrow 0} \sup _{(p, b) \in \mathcal{E}(\Delta)} \Pi^{\Delta}(p, b)
$$

The analysis of the continuous time limit is more tractable and we will justify our approach by providing approximations through discrete time equilibria. An alternative approach is to set up the model directly in continuous time. This alternative approach, however, has unresolved conceptual issues regarding the definition of strategies and equilibrium concepts in continuous time games of perfect monitoring, which are beyond the scope of this paper 13

It is worth noting that when $n=1$, our setup reduces to the model of Ausubel and Deneckere (1989) where the seller is restricted to post prices. They show that there are two classes of equilibria. The first class are weak-Markov equilibria. These equilibria satisfy the Coase conjecture and have a limit profit of zero. The second class are non-Markov reputational equilibria. Each reputational equilibrium consists of an equilibrium price path and a punishment which is taken from a weakMarkov equilibrium. The equilibrium price path starts with an arbitrary initial price and may decline at an arbitrarily slow rate as $\Delta$ becomes small. A deviation from the equilibrium path by the seller is deterred by a threat to switch to the weak-Markov equilibrium. By adjusting the initial price and the rate of the decline, the seller can attain any profit between zero and the static monopoly profit. In other words, in the bargaining set up, $\Pi^{*}$ is the monopoly profit. Even though the seller lacks full commitment power, there are equilibria where she can attain a profit arbitrarily close to what she could get with full commitment power.

From now on, we assume that $n \geq 2$. With multiple buyers the model behaves quite differently. Weak-Markov equilibria still exist and they satisfy the Coase conjecture in the sense that the seller looses her monopoly power in these equilibria (see Section 5), but there is an important difference to the case of one buyer: With multiple buyers, the seller always earns a strictly positive profit. In particular, the seller always has the option to run a second price auction without reserve price to end the game, which yields a strictly positive profit (see Lemma 3). This imposes a lower bound on the punishment that can be used to support an equilibrium with higher profits. As a result, $\Pi^{*}$ must be lower than the profit under full commitment since this profit could only be achieved by a constant reserve price equal to the static optimal reserve price, which would require a punishment that reduces the seller's profit to zero. Therefore, finding $\Pi^{*}$ is a mechanism design

\footnotetext{
${ }^{12}$ See Fudenberg and Tirole (1991) for the definition of PBE in finite games. The extension to infinite games is straightforward.

${ }^{13}$ See Bergin and MacLeod (1993) and Fuchs and Skrzypacz (2010) for related discussions.
} 
problem. Moreover, the equilibrium construction is significantly more complex than in the bilateral bargaining setting where arbitrary price paths can be implemented.

In order to characterize the maximal profit $\Pi^{*}$, we start with three basic lemmas. The first lemma states that in every equilibrium of the game, all buyers play pure strategies that can be characterized by cutoffs. In particular, a buyer's strategy in period $t$ can be summarized by a cutoff $\beta_{t}$ which depends only on the sequence of reserve prices announced up to period $t \sqrt{14}$ Given our symmetry restriction, this cutoff $\beta_{t}$ is the same for all buyers.

Lemma 1. Let $(p, b) \in \mathcal{E}(\Delta)$. Then, for each $t=0, \Delta, 2 \Delta, \ldots$, there exists a function $\beta_{t}: H_{t} \times$ $[0,1] \rightarrow[0,1]$ such that every bidder with valuation above $\beta_{t}\left(h_{t}, p_{t}\right)$ places a valid bid and every bidder with valuation below $\beta_{t}\left(h_{t}, p_{t}\right)$ waits if the seller announces reserve price $p_{t}$ at history $h_{t}$.

Proof. The proof follows from the same arguments as the proof of Lemma 10.1 in Fudenberg and Tirole (1991).

The second lemma shows that, although the seller is allowed to randomize, randomization on the equilibrium path is not necessary to attain the optimal profit.

Lemma 2. For every equilibrium $(p, b) \in \mathcal{E}(\Delta)$, there exists an equilibrium $\left(p^{\prime}, b^{\prime}\right) \in \mathcal{E}(\Delta)$ in which the seller does not randomize on the equilibrium path and achieves a profit at least as high as in $(p, b)$.

Proof. All omitted proofs can be found in the Appendix.

Lemmas 1 and 2 imply that the maximal profit of the seller can be achieved in an equilibrium that implements a deterministic allocation rule (up to tie-breaking).

The third lemma shows the seller can ensure a continuation profit no smaller than the profit of an efficient auction. This restriction on continuation payoffs plays a critical role in our analysis.

Lemma 3. Fix any equilibrium $(p, b) \in \mathcal{E}(\Delta)$ and any history $h_{t}$. If the seller announces the reserve price $p_{t}=0$ at this history (this may not be an equilibrium strategy), then every bidder bids their true values and the game ends.

Next, we present several technical assumptions on the distribution function $F$ that are necessary for our subsequent analysis. As is standard in the literature, we assume that the distribution $F$ has the monotone virtual value (monotone marginal revenue) property.

Assumption A1 $J(v):=v-(1-F(v)) / f(v)$ is strictly increasing on $[0,1]$.

In addition, we will make two regularity assumptions on the distribution $F$. First, we need a mild assumption on the tail of the distribution:

\footnotetext{
${ }^{14}$ This result is an analog of a result by Fudenberg, Levine, and Tirole (1985).
} 
Assumption A2 $\lim _{v \rightarrow 0}\left(f^{\prime}(v) v\right) / f(v)$ exists and is finite.

This assumption is satisfied, for example, if the density function $f$ is bounded away from 0 and has a bounded derivative. It is also satisfied for a much more general class of distributions which includes densities with $f(0)=0$. For instance, consider a family of power function distributions $F(v)=v^{k}$ with $k>0$. For this family, we have $\left(f^{\prime}(v) v\right) / f(v)=k-1$ independent of $v$.

Assumption A3 There exist $0<M \leq 1 \leq L<\infty$ and $\alpha>0$ such that $M v^{\alpha} \leq F(v) \leq L v^{\alpha}$ for all $v \in[0,1]$.

This regularity assumption is adopted from Ausubel and Deneckere (1989) whose bargaining model can be viewed as a special case of our auction model. Ausubel and Deneckere (1989) use this assumption to establish the existence of weak-Markov equilibria and prove the uniform Coase conjecture. We extend these two basic results to our auction setting.

For one of our results, we need the following more restrictive assumption. Later, we will be explicit about when this assumption is used.

Assumption A4 The revenue function $v(1-F(v))$ is concave on $[0,1]$.

Assumption A4 is equivalent to assuming that $J(v) f(v)$ is increasing. It is also equivalent to $\left(f^{\prime}(v) v\right) / f(v)>-2$. For example, the family of power function distributions given by $F(v)=v^{k}$ satisfies Assumption A4 for all $k>0$.

\section{A Mechanism Design Approach to Limited Commitment}

To solve for $\Pi^{*}$, we consider an auxiliary mechanism design problem in which the seller has full commitment power but is restricted by a sequential rationality constraint. We formulate the auxiliary problem in continuous time, and consider direct mechanisms in which all buyers make a single report of their valuations at time zero. Depending on the profile of reports, a mechanism selects a winner, a trading time, and a payment. In our definition, direct mechanisms must satisfy a number of properties inherited from equilibria in the original game. We first give an overview of these properties before we formally define the mechanism design framework.

Basic Properties of Direct Mechanisms: The first set of constraints are derived from properties of buyer symmetric equilibria in a sequence of second price auctions 15 The allocation rule must be symmetric, only the buyer with the highest type can win the object, buyers with high valuations trade earlier than buyers with lower valuations, and only the winner makes a payment. Since we are interested in the limits of equilibrium outcomes, we will impose these restrictions on the trading time, winner selection, and payment rule.

\footnotetext{
${ }^{15}$ If we would study a general mechanism design problem, we would not have to impose these conditions on the allocation rules and payment rules that can be implemented in equilibrium.
} 
Incentive Constraints for the Buyers: Since we assume that buyers report their types at time zero, their incentives only depend on the discounted trading probabilities and the discounted payments. Therefore, buyer incentive constraints in the auxiliary problem are identical to incentive compatibility constraints from static mechanism design problems, except that winning probabilities and payments are replaced by discounted winning probabilities and discounted payments.

Sequential Rationality for the Seller (The Payoff Floor Constraint): Finally, in the original game, the seller always has the option to run an efficient auction to end the game. This imposes a necessary lower bound on the continuation profit on the equilibrium path. Therefore, we impose the constraint that the expected discounted continuation profit of the seller is at least as high as the revenue of an efficient auction for the given posterior at each point in time. The explicit specification of trading times in our mechanisms is necessary for the formulation of this constraint, because it allows us to construct the posterior distribution for each point in time. The payoff floor constraint is the crucial element in the auxiliary problem which distinguishes it from a standard mechanism design problem under full commitment.

In the following, we define the trading time, winner selection rule and payment rule, as well as the constraints formally.

\subsection{Direct Mechanisms}

To define a direct mechanism, we specify, for each type profile $\left(v^{i}, v^{-i}\right)$ and for each buyer $i$, the trading time $\tau^{i}:[0,1]^{n} \rightarrow[0,+\infty]$ and corresponding payment $\pi^{i}:[0,1]^{n} \rightarrow \mathbb{R}$. If $\tau^{i}\left(v^{i}, v^{-i}\right)<\infty$, buyer $i$ is awarded the object at time $\tau^{i}\left(v^{i}, v^{-i}\right)$ and the payment $\pi^{i}\left(v^{i}, v^{-i}\right)$ is also made at time $\tau^{i}\left(v^{i}, v^{-i}\right)$. If $\tau^{i}\left(v^{i}, v^{-i}\right)=\infty$, then $i$ is not awarded the object for the given type profile.

Motivated by the properties of equilibria in the discrete time game, we impose the following restrictions on the set of direct mechanisms $(\tau, \pi)$ :

1. Only the highest bidder can win: $\tau^{i}\left(v^{i}, v^{-i}\right)=\infty$ if $v^{i}<v^{(n)}$. Ties are broken randomly ${ }^{16}$

2. Only the winner pays: $\pi^{i}\left(v^{i}, v^{-i}\right)=0$ if $v^{i}<v^{(n)}$.

3. The mechanism is symmetric: $\tau^{i}\left(v^{i}, v^{-i}\right)=\tau^{\sigma(i)}\left(v^{\sigma(1)}, \ldots, v^{\sigma(n)}\right)$ and $\pi^{i}\left(v^{i}, v^{-i}\right)=\pi^{\sigma(i)}\left(v^{\sigma(1)}, \ldots, v^{\sigma(n)}\right)$ for every permutation $\sigma$.

4. The winner's trading time only depends on his own type: $\tau^{i}\left(v^{i}, v^{-i}\right)=\tau^{i}\left(\hat{v}^{i}, \hat{v}^{-i}\right)$ if $v^{i}=$ $v^{(n)}=\hat{v}^{(n)}=\hat{v}^{i}$.

5. Higher types trade earlier: if $v^{i}=v^{(n)} \leq \hat{v}^{(n)}=\hat{v}^{i}$, then $\tau^{i}\left(\hat{v}^{i}, \hat{v}^{-i}\right) \geq \tau^{i}\left(v^{i}, v^{-i}\right)$.

\footnotetext{
${ }^{16}$ We do not formally include the randomization in the definition of $\tau$ because ties occur with probability zero.
} 
6. If buyer $i$ wins, he pays $\pi^{i}\left(v^{i}, v^{-i}\right)$ at time $\tau^{i}\left(v^{i}, v^{-i}\right)$.

The tuple $(\tau, \pi)$, together with restriction 6 and the tie-breaking rule, fully specifies the allocation and the payment rule of a direct mechanism. Therefore, with slight abuse of notation, we call $(\tau, \pi)$ a direct mechanism. For a given direct mechanism $(\tau, \pi)$, the discounted trading probability of buyer $i$ for a given type profile $\left(v^{i}, v^{-i}\right)$ is

$$
q^{i}\left(v^{i}, v^{-i}\right):=\frac{1}{\#\left\{j: v^{j}=v^{(n)}\right\}} e^{-r \tau^{i}\left(v^{i}, v^{-i}\right)} .
$$

The discounted payment of bidder $i$ is given by

$$
m^{i}\left(v^{i}, v^{-i}\right):=q^{i}\left(v^{i}, v^{-i}\right) \pi^{i}\left(v^{i}, v^{-i}\right)
$$

The symmetry and monotonicity restrictions on trading time $\tau^{i}$ enable us to describe $\tau^{i}$ by a single function $T:[0,1] \rightarrow[0, \infty]$, which describes the trading time of the buyer with the highest type.

Lemma 4. Let $(\tau, \pi)$ be a direct mechanism. Then there exists a non-increasing function $T$ : $[0,1] \rightarrow[0,+\infty]$ such that the discounted trading time derived from $\tau$ satisfies

$$
q^{i}\left(v^{i}, v^{-i}\right)=\frac{\mathbf{1}_{\left\{v^{i}=v^{(n)}\right\}}}{\#\left\{j: v^{j}=v^{(n)}\right\}} e^{-r T\left(v^{i}\right)} .
$$

\subsection{Buyer Incentive Compatibility}

For a given direct mechanism $(\tau, \pi)$, we define the expected discounted trading probability of type $v^{i}$ as $Q^{i}\left(v^{i}\right):=E_{v^{-i}}\left[q^{i}\left(v^{i}, v^{-i}\right)\right]$, and the expected discounted payment as $M^{i}\left(v^{i}\right):=$ $E_{v^{-i}}\left[m^{i}\left(v^{i}, v^{-i}\right)\right]$. A direct mechanism $(\tau, \pi)$ is incentive compatible if the following (static) incentive constraint is satisfied for all $v^{i}, \hat{v}^{i} \in[0,1]$,

$$
v^{i} Q^{i}\left(v^{i}\right)-M^{i}\left(v^{i}\right) \geq v^{i} Q^{i}\left(\hat{v}^{i}\right)-M^{i}\left(\hat{v}^{i}\right)
$$

We denote the payoff of buyer $i$ under $(\tau, \pi)$ by $U^{i}\left(v^{i}\right)=v^{i} Q^{i}\left(v^{i}\right)-M^{i}\left(v^{i}\right)$. Note that by Restriction 1 , a buyer with type $v^{i}=0$ gets the object with probability 0 , and by Restriction 2 , his payment is zero. Therefore, we must have $U^{i}(0)=0$. Using standard techniques Krishna, 2002 ), we obtain the envelope formula for the buyer's payoff as a necessary condition for incentive compatibility,

$$
U^{i}\left(v^{i}\right)=\int_{0}^{v^{i}} Q^{i}(x) d x
$$

Together with monotonicity of $Q^{i}$, this condition is sufficient for incentive compatibility. Instead of imposing monotonicity on $Q^{i}$, however, we note that (3.1) implies that the monotonicity constraint can be imposed directly on the trading time function $T$. 
Lemma 5. Let $T:[0,1] \rightarrow[0,+\infty]$ be non-increasing. Then there exists an incentive compatible direct mechanism $(\tau, \pi)$ with

$$
\tau^{i}\left(v^{i}, v^{-i}\right)=T\left(v^{i}\right) \cdot \mathbf{1}_{\left\{v^{i}=v^{(n)}\right\}} \cdot
$$

Next, we show that every incentive compatible direct mechanism satisfying Restrictions 1-6 corresponds to a sequence of cutoffs $\left(v_{t}\right)_{t \in \mathbb{R}}$ that describe the allocation rule and a sequence of reserve prices $\left(p_{t}\right)_{t \in \mathbb{R}}$ that implements this allocation rule. Let us define

$$
v_{t}:=\sup \{v \mid T(v) \geq t\}
$$

That is, $v_{t}$ is the highest type that does not trade before time $t$. Since all buyers with types $v>v_{t}$ trade before $t$, the posterior distribution at $t$, conditional on the event that the object has not yet been allocated, is given by the truncated distribution $F\left(v \mid v \leq v_{t}\right)$. Therefore, we call $v_{t}$ the posterior at time $t$. Generally, $v_{t}$ is continuous from the left, and since it is non-increasing, the right limit exists everywhere. We will denote the right limit at $t$ by

$$
v_{t}^{+}:=\lim _{s \searrow t} v_{s}
$$

For each $t, v_{t}^{+}$is the highest type in the posterior after time $t$ if the object is not yet sold.

Next, we define a sequence of prices $\left(p_{t}\right)_{t \in \mathbb{R}}$ which implement cutoffs $\left(v_{t}\right)_{t \in \mathbb{R}}$. This means at time $t$, type $v_{t}^{+}$is indifferent between buying immediately at price $p_{t}$ and waiting 17 All higher types strictly prefer to bid before or at time $t$, whereas all lower types strictly prefer to wait.

Lemma 6. Let $T:[0,1] \rightarrow[0,+\infty]$ be non-increasing and $\left(v_{t}\right)_{t \in \mathbb{R}}$ the corresponding sequence of cutoffs. Then the following sequence of prices implements $\left(v_{t}\right)_{t \in \mathbb{R}}$ :

$$
p_{t}=v_{t}^{+}-e^{r T\left(v_{t}^{+}\right)} \int_{0}^{v_{t}^{+}} e^{-r T(v)}\left(\frac{F(v)}{F\left(v_{t}^{+}\right)}\right)^{n-1} d v
$$

Notice that if the cutoff path is steep (i.e., the cutoff $v_{t}$ declines quickly) then the price path $p_{t}$ is also steep. A steeper cutoff path also implies that the payoff for the cutoff type, $v_{t}^{+}-p_{t}$, is larger because waiting becomes more attractive. If $v_{t}$ is differentiable, we have $v_{t}^{+}=v_{t}$, and we can use a change of variables to obtain an expression for $p_{t}$ in terms of $v_{t}$ only:

$$
p_{t}=v_{t}+\int_{t}^{\infty} e^{-r(s-t)}\left(\frac{F\left(v_{s}\right)}{F\left(v_{t}\right)}\right)^{n-1} \dot{v}_{s} d s
$$

\footnotetext{
${ }^{17}$ Note that $v_{t}^{+}$is the infimum of all types that trade at time $t$. Therefore, if the reserve price at time $t$ is $p_{t}$, the buyer with valuation $v_{t}^{+}$will pay price $p_{t}$ if she makes a truthful bid at time $t$ and this bid wins.
} 


\subsection{The Payoff Floor Constraint}

The seller chooses a mechanism to maximize her expected revenue. Using the envelope condition, we can write the expected discounted payment received from an individual buyer as follows:

$$
E_{v^{i}}\left[M^{i}\left(v^{i}\right)\right]=\int_{0}^{1} Q^{i}\left(v^{i}\right) J\left(v^{i}\right) d F\left(v^{i}\right)=E_{\left(v^{i}, v^{-i}\right)}\left[q^{i}\left(v^{i}, v^{-i}\right) J\left(v^{i}\right)\right]
$$

Therefore, the total expected discounted revenue is given by

$$
\Pi=\sum_{i} E_{v^{i}}\left[M^{i}\left(v^{i}\right)\right]=\sum_{i} E_{\left(v^{i}, v^{-i}\right)}\left[q^{i}\left(v^{i}, v^{-i}\right) J\left(v^{i}\right)\right]=\int_{0}^{1} e^{-r T(x)} J(x) d F^{(n)}(x),
$$

where the last step follows from Lemma 4.

Without further constraints, the following trading time function

$$
T^{M}(v):= \begin{cases}0 & \text { if } J(v) \geq 0 \\ \infty & \text { if } J(v)<0\end{cases}
$$

maximizes the seller's expected discounted revenue $\Pi$. The trading time $T^{M}$ corresponds to the allocation rule of the revenue-maximizing auction with full commitment as characterized by Myerson (1981). The object is allocated to the buyer with the highest valuation if his virtual valuation is non-negative. Otherwise the object is not sold. The corresponding cutoff path is constant and satisfies $J\left(v_{t}\right)=0$ for all $t>0$. By equation (3.3), the seller must use the constant path of reserve prices given by $J\left(p_{t}\right)=0$ in order to implement this allocation rule.

Since the seller has limited commitment power, not every incentive compatible direct mechanism describes the limit of equilibrium outcomes of our original game. In particular, the seller can always run a second-price auction without reserve price to end the game at any point in time. Therefore, we formulate an additional constraint that will capture the spirit of the seller's sequential rationality. We require that for all $t \geq 0$,

$$
\sum_{i} E_{\left(v^{i}, v^{-i}\right)}\left[e^{r t} m^{i}\left(v^{i}, v^{-i}\right) \mid v^{(n)} \leq v_{t}\right] \geq \Pi^{E}\left(v_{t}\right)
$$

We dub this condition the "payoff floor" constraint. The right-hand side of the constraint, $\Pi^{E}\left(v_{t}\right)$, is the profit of an efficient auction when the type distribution is $F\left(v \mid v \leq v_{t}\right)$. On the left-hand side of (3.5), we have the continuation profit at time $t$ if the object has not been allocated yet.

One solution that always satisfies this constraint is the direct mechanism defined by the efficient allocation rule:

$$
T^{E}(v)=0, \quad \forall v \in[0,1]
$$


This solution corresponds to a second-price auction with reserve price $p_{t}=0$ at time $t=0$. Since we have $v_{t}=0$ for all $t>0$, the payoff floor constraint is trivially satisfied for $t>0$. For $t=0$, the constraint is trivially satisfied because we have the profit of an efficient auction on both sides.

In order to maximize revenue subject to (3.5), we need a more tractable formulation. If we define

$$
\begin{gathered}
J_{t}(v):=v-\frac{F\left(v_{t} \mid v \leq v_{t}\right)-F\left(v \mid v \leq v_{t}\right)}{f\left(v \mid v \leq v_{t}\right)}=v-\frac{F\left(v_{t}\right)-F(v)}{f(v)}, \\
F_{t}(x):=\frac{F(x)}{F\left(v_{t}\right)}, \quad \text { and } \quad F_{t}^{(n)}(x):=\frac{F^{n}(x)}{F^{n}\left(v_{t}\right)}
\end{gathered}
$$

the right-hand side 3.5$)$ is equal to

$$
\Pi^{E}\left(v_{t}\right)=\int_{0}^{v_{t}} J_{t}(x) d F_{t}^{(n)}(x)
$$

In order to reformulate the continuation profit on the left-hand side, we now show that under Restrictions 1-6, our definition of incentive compatibility of mechanisms in 3.2 also implies incentive compatibility for all $t$.

Lemma 7. Let $(\tau, \pi)$ be an incentive compatible direct mechanism. Then for all $t$ and all $v^{i}, \hat{v}^{i} \in$ $\left[0, v_{t}\right]$, we have

$$
\begin{aligned}
E_{v^{-i}}\left[q^{i}\left(v^{i}, v^{-i}\right) \mid v^{(n)} \leq\right. & \left.v_{t}\right] v^{i}-E_{v^{-i}}\left[m^{i}\left(v^{i}, v^{-i}\right) \mid v^{(n)} \leq v_{t}\right] \\
& \geq E_{v^{-i}}\left[q^{i}\left(\hat{v}^{i}, v^{-i}\right) \mid v^{(n)} \leq v_{t}\right] v^{i}-E_{v^{-i}}\left[m^{i}\left(\hat{v}^{i}, v^{-i}\right) \mid v^{(n)} \leq v_{t}\right] .
\end{aligned}
$$

Although buyers do not submit reports at time $t>0$, we have obtained a condition that formally resembles an incentive constraint at time $t$. This is not surprising. A buyer with valuation $v^{i} \leq v_{t}$ is only interested in the allocations at time $t$ or later because he cannot get the object before $t$. Moreover, a report $v^{i} \leq v_{t}$ will not affect possible allocations to other buyers before time $t$. Therefore, in a hypothetical situation where all buyers with type $v^{i} \leq v_{t}$ are asked to report at time $t$, their incentives are exactly the same as at time zero. In this sense (3.6) can be interpreted as a period $t$ incentive constraint.

Using (3.6), we can rewrite the left-hand side of (3.5) as

$$
\begin{aligned}
& \sum_{i} E_{\left(v^{i}, v^{-i}\right)}\left[e^{r t} m^{i}\left(v^{i}, v^{-i}\right) \mid v^{(n)} \leq v_{t}\right] \\
= & \sum_{i} \int_{\left[0, v_{t}\right]^{n-1}}\left[\int_{0}^{v_{t}} e^{-r\left[\tau^{i}\left(v^{i}, v^{-i}\right)-t\right]} \pi^{i}\left(v^{i}, v^{-i}\right) d F_{t}\left(v^{i}\right)\right] d F_{t}\left(v^{-i}\right) \\
= & \int_{0}^{v_{t}} e^{-r(T(x)-t)} J_{t}(x) d F_{t}^{(n)}(x),
\end{aligned}
$$

where we have used the envelope condition and Lemma 4 to obtain the last line. Multiplying both 
sides of 3.5 by $F^{n}\left(v_{t}\right)$, we obtain the following formulation for the payoff floor constraint.

$$
\int_{0}^{v_{t}} e^{-r(T(x)-t)} J_{t}(x) d F^{(n)}(x) \geq \int_{0}^{v_{t}} J_{t}(x) d F^{(n)}(x) .
$$

\subsection{The Auxiliary Problem}

To summarize, we can formulate the auxiliary problem as the following mechanism design problem:

$$
\sup _{T:[0,1] \rightarrow[0,+\infty]} \int_{0}^{1} e^{-r T(x)} J(x) d F^{(n)}(x)
$$

s.t. $T$ is non-increasing,

$$
\forall t \geq 0: \int_{0}^{v_{t}} e^{-r(T(x)-t)} J_{t}(x) d F^{(n)}(x) \geq \int_{0}^{v_{t}} J_{t}(x) d F^{(n)}(x) .
$$

In Section 5, we will show the close linkage between the auxiliary problem with full commitment and our original problem with limited commitment, which justifies our mechanism design approach. In particular, we will show that (a) the trading time functions obtained as limits of equilibrium outcomes (as $\Delta \rightarrow 0$ ) must lie in the feasible set of the auxiliary problem, and (b) the optimal solutions to the auxiliary problem we construct in Section 4, are limits of equilibrium outcomes in the discrete time game (as $\Delta \rightarrow 0$ ). Therefore, in order to find the revenue-maximizing equilibria in our original problem, it is adequate to solve the auxiliary problem.

We have already seen above that the feasible set of the auxiliary problem is non-empty because it contains the efficient auction $T^{E}$. As we will see in the next section, there may also be feasible but inefficient solutions where the allocations to low types are delayed. The object cannot, however, remain unsold forever with positive probability. In particular, the full commitment solution is not feasible.

Theorem 1. Suppose $T$ is a feasible solution to the auxiliary problem. Then $T(v)<\infty$ for all $v>0$. In particular, the optimal auction under full commitment $T^{M}$ is not feasible.

The intuition is straightforward. If $T(v)=\infty$ for all $v \in[0, \bar{v}]$, where $\bar{v}>0$, then the seller's posterior converges to $v_{t}=\bar{v}$ as $t \rightarrow \infty$. With $v_{t}$ converging to $\bar{v}$, the probability that a trade takes place after time $t$ vanishes. Therefore, the continuation revenue converges to zero. This, however, violates the payoff floor constraint because the profit of an efficient auction is strictly positive as $v_{t} \geq \bar{v}>0$.

To conclude this section, we note first that standard techniques can be used to show that an optimal solution to the auxiliary problem exists.

Proposition 1. An optimal solution to the auxiliary problem exists.

Second, we note that the value of the auxiliary problem does not depend on $r$. 
Proposition 2. The value of the auxiliary problem is independent of $r>0$. Moreover, if $T$ is a feasible solution for given $r$, then $\hat{T}(v):=(r / \hat{r}) T(v)$, defines a feasible solution for the auxiliary problem for $\hat{r}>0$. The profit under $\hat{r}$ and $\hat{T}$ is equal to the profit under $r$ and $T$.

In continuous time, $r$ only determines how much delay is needed to reduce the value of an allocation or a payment by a certain amount, but a change in $r$ does not affect the possibility of destroying value by delaying an allocation. Thus, a change in $r$ is equivalent to a change in the unit of measurement for time, which is irrelevant if $t$ is a continuous variable.

\section{Revenue-Maximizing Auctions}

In order to obtain optimal solutions to the auxiliary problem, we first argue that it is revenueimproving for the seller to use positive reserve prices whenever this is credible. In other words, the efficient auction $\left(T^{E}\right)$ is optimal if and only if it is the only feasible solution to the auxiliary problem. It is clear that any feasible solution yields a profit that is at least as high as the profit of the efficient auction. Otherwise, the payoff floor constraint would be violated at $t=0$. The following proposition shows that if positive reserve prices are feasible, that is, the feasible set includes a solution with delayed trade for low types, then the seller can achieve a strictly higher revenue than in the efficient auction.

Proposition 3. An efficient auction $\left(T^{E}\right)$ is an optimal solution to the auxiliary problem if and only if it is the only feasible solution.

To get an intuition for this result, compare the efficient auction in which all types trade at time zero, to an alternative feasible solution in which only the types in $\left(v_{0}^{+}, 1\right]$ trade at time zero, where $v_{0}^{+}<1.18$ There are two effects that determine how the profits of these two solutions are ranked. First, in the alternative, the trade of low types is delayed, which creates an inefficiency. Second, the delay for the low types reduces information rents for higher types. We must argue that the total reduction in information rents exceeds the inefficiency, so that the ex-ante profit is higher under the alternative solution. We first consider the reduction in information rents only for the types in $\left[0, v_{0}^{+}\right]$. This is what matters for the continuation profit at time $0^{+}$, that is, right after the initial trade. Feasibility implies that the reduction in information rents for the types in $\left[0, v_{0}^{+}\right]$must already (weakly) exceed the revenue loss from inefficiency. Otherwise, the continuation profit at $0^{+}$would be smaller than the profit from an efficient auction given the posterior $\left[0, v_{0}^{+}\right]$, and thus the payoff floor constraint would be violated. If we now include the types in $\left(v_{0}^{+}, 1\right]$ in the comparison, we must add the reduction in information rents for these types but there is no additional inefficiency because these types trade at time zero in both solutions. Therefore, the total

\footnotetext{
${ }^{18}$ In the proof of Proposition 3 we show that we can always construct a feasible solution with $0<v_{0}^{+}<1$, if there exists any feasible solution that differs from the efficient auction.
} 
reduction in information rents is strictly higher than the inefficiency, and the ex-ante profit under the alternative is strictly higher than under the efficient auction.

Proposition 3 implies that in order to show that the efficient auction is optimal, it suffices to show that it is the unique feasible solution. It turns out that this depends solely on the lower tail of the type distribution and the number of buyers. To make this precise, we define

$$
\phi:=\lim _{v \rightarrow 0} \frac{f^{\prime}(v) v}{f(v)}
$$

Remark 1. By Assumption A2, $\phi$ is well-defined and finite. Moreover, we can show that every distribution for which $\phi$ is well-defined satisfies $\phi \geq-1$. To understand this observation, consider distributions for which $\frac{f^{\prime}(v) v}{f(v)}$ is independent of $v$ in a neighborhood of zero. If this is the case, then the distribution function $F$ must look like a CRRA utility function with relative risk aversion coefficient $\rho=-\phi$. It is well-known that for $\rho \geq 1$, CRRA utility functions are unbounded at zero. Hence, we must have $\phi>-1$ to obtain a distribution function. For general distributions, we show in the Appendix that $\phi \geq-1$, and rule out the knife-edge case of $\phi=-1$ by assumption 19

Our first main result is that there exists a distribution-specific cutoff

$$
\bar{N}(F):=1+\frac{\sqrt{2+\phi}}{1+\phi}
$$

such that the efficient auction is optimal if and only if the number of bidders $n$ exceeds $\bar{N}(F)$.

Theorem 2. Suppose $\phi>-1$.

(i) If $n<\bar{N}(F)$, the efficient auction is not optimal and the optimal solution has strictly positive reserve prices.

(ii) If $n>\bar{N}(F)$, the efficient auction is optimal and it is the only feasible solution for the auxiliary problem.

If $F$ is uniform, then $\phi=0$ and the cutoff is $1+\sqrt{2}$. Therefore, the efficient auction is optimal if there are three or more bidders. If there are two bidders, positive reserve prices are feasible and the seller can achieve a higher profit than in the efficient auction. Under mild assumptions on the type distribution, this property holds more generally as the following corollary shows. The second part of the corollary gives a sufficient condition under which $\phi=1$ so that $\bar{N}(F)=1+\sqrt{3} / 2<2.20$

Corollary 1. (i) If the density satisfies $f(0)>0$ and has a bounded derivative at 0 , then the efficient auction is optimal if $n \geq 3$. If $n \leq 2$, the seller maximizes her revenue by using positive reserve prices.

\footnotetext{
${ }^{19}$ This is a mild assumption but it is not without loss of generality. For example, for the distribution function $F(v)=v^{(\ln (1 / v))^{-(1 / 2)}}$, we have $\phi=-1$. We thank Yuliy Sannikov for providing this example.

${ }^{20}$ We thank Bernard Salanié for pointing out this case.
} 
(ii) If the density is twice continuously differentiable at zero, $f(0)=0$ and $f^{\prime}(0) \neq 0$, then the efficient auction is optimal for all $n \geq 2$.

Despite the observation that the cutoff is often very low, there is also a natural class of distributions, for which $\bar{N}(F)$ can become arbitrarily large (see Example 2 below).

At first glance, it seems surprising that the existence of feasible solutions that differ from the efficient auction only depends on the lower tail of the distribution. The intuition is as follows. Notice that the seller's posterior is a truncation from above of the original distribution. Therefore, the tail of the distribution determines the set of equilibria in subgames which start after sufficiently many periods. Suppose the tail of the distribution allows multiple equilibria in every subgame starting in period $t+\Delta$. Then, there are also multiple equilibria in any subgame starting at $t$. In contrast, if the tail of the distribution pins down a unique continuation equilibrium for all possible histories after sufficiently many periods, then there is a unique equilibrium in the whole game. Therefore, the existence of multiple equilibria, and thus the multiplicity of solutions to the auxiliary problem, only depend on the tail of the distribution.

When the number of buyers is small, that is $n<\bar{N}(F)$, Theorem 2 shows that the seller uses strictly positive reserve prices to maximize her revenue. Our second main result characterizes the optimal selling strategy for this case. If we impose Assumption A4, the payoff floor constraint binds for all $t>0$ at the optimal solution.

Proposition 4. If $v(1-F(v))$ is concave, then for every optimal solution, the payoff floor constraint binds for all $t>0$.

If the payoff floor constraint binds everywhere, then the cutoff $v_{t}$ is twice continuously differentiable and satisfies the following ordinary differential equation (see Lemmas 12 and 13 in Appendix A.3.1):

$$
\frac{\ddot{v}_{t}}{\dot{v}_{t}}+g\left(v_{t}\right) \dot{v}_{t}+r=0
$$

where

$$
g\left(v_{t}\right)=\frac{f^{\prime}\left(v_{t}\right)}{f\left(v_{t}\right)}-\frac{\left\{v_{t}\left(F\left(v_{t}\right)\right)^{n-1}-2 \int_{0}^{v_{t}}(F(v))^{n-1} d v\right\} f\left(v_{t}\right)}{(n-1) \int_{0}^{v_{t}}\left[F\left(v_{t}\right)-F(v)\right](F(v))^{n-2} f(v) v d v} .
$$

If $n<\bar{N}(F)$, this ODE has a decreasing solution (see Lemma 15 in Appendix A.3.2 and we have the following result:

Theorem 3. If $v(1-F(v))$ is concave and $n<\bar{N}(F)$, the optimal solution to the auxiliary problem is given by a cutoff path that starts with $v_{0}^{+}>0$ and satisfies

$$
\dot{v}_{t}=-\int_{0}^{v_{t}} r e^{-\int_{v}^{v_{t}} g(x) d x} d v
$$


To illustrate this result, we discuss several examples in which we obtain a closed-form solution for the ODE in 4.1) and the initial value $v_{0}^{+}$. Generally, the optimal initial value can be computed by inserting the solution to the ODE into the seller's objective function and maximizing over $v_{0}^{+}$. For some distributions this can be done in closed form. We begin with the uniform distribution.

Example 1 (Uniform Distribution). Suppose the buyers' types are uniformly distributed: $v \sim$ $U[0,1]$. Then $\phi=0$ and $\bar{N}(F)=1+\sqrt{2}$. It follows from Theorem 2 that the efficient auction is optimal if $n \geq 3$, and the efficient auction is not optimal if $n=2$. In the case of $n=2$, the ODE in (4.1) becomes

$$
\ddot{v}_{t}+r \dot{v}_{t}=0 \text {. }
$$

The solution to this $\mathrm{ODE}$ is $v_{t}=v_{0}^{+} e^{-r t}$, and the corresponding trading time is given by

$$
T(v)= \begin{cases}0 & \text { if } v>v_{0}^{+} \\ -\left(\ln v-\ln v_{0}^{+}\right) / r & \text { if } v \leq v_{0}^{+}\end{cases}
$$

By inserting $T(v)$ into the seller's objective function and maximizing over $v_{0}^{+}$, we obtain the optimal cutoff $v_{0}^{+}=2 / 3$. We can then obtain the optimal sequence of reserve prices $p_{t}=(4 / 9) e^{-r t}$ from Lemma 6. Since both $v_{t}$ and $p_{t}$ are strictly positive for all $t>0$ and converge to zero as $t \rightarrow \infty$, the object is sold eventually but the game may not end in finite time. This is a general feature of the optimal solution for any distribution if $n<\bar{N}(F)$.

Example 2 (Power Function Distribution). Suppose the buyers' valuations are distributed according to $F(v)=v^{k}$ with support $[0,1]$ and $k>0$. Then, $\phi=k-1$ and $\bar{N}(F)=1+\sqrt{1+k} / k$. The ODE in 4.1) becomes

$$
\frac{\ddot{v}_{t}}{\dot{v}_{t}}+\kappa \frac{\dot{v}_{t}}{v_{t}}+r=0
$$

where

$$
\kappa=(k-1)-\frac{(n k-k-1)(n k+1)}{(n-1) k} .
$$

The solution is given by

$$
v_{t}=v_{0}^{+} e^{-\rho t}
$$

where $\rho=r /(1+\kappa)$. Therefore, if $\kappa>-1$, then $0<\rho<r$, and the solution to the ODE is decreasing. This corresponds to the case that $n<\bar{N}(F)$. Indeed, it is easy to verify that $\kappa>-1$ if and only if $n<\bar{N}(F)$. In contrast, if $\kappa<-1$, or equivalently $n>\bar{N}(F)$, the solution to the ODE is increasing, so that the binding payoff floor constraint does not yield a feasible solution. This is not surprising since by Theorem 2 the efficient auction is optimal and also the only feasible solution. To illustrate, we can set $n=3$ and $k=1$ so that $F$ is uniform. Then $\kappa=-2$ and the solution of the ODE becomes $v_{t}=v_{0}^{+} e^{r t}$ which is increasing. 
In the remainder of this section, we give an overview of the proofs of Theorems 2 and 3 . The formal proofs can be found in Appendix A.3. Both results follow from the analysis of the binding payoff floor constraint. As the first step, we show that if $T$ is a feasible solution for which the payoff floor constraint is binding for all $t>0$, then $v_{t}$ is twice continuously differentiable and satisfies the ODE in 4.1 (see Lemmas 12 and 13 in Appendix A.3.1. Second, this ODE yields a feasible solution to the auxiliary problem if $n<\bar{N}(F)$ (see Lemma 15 in Appendix A.3.2. Proposition 3. together with the construction of a feasible solution that is different from the efficient auction (via the binding payoff floor constraint), implies part (i) of Theorem 2, If $n>\bar{N}(F)$ the solution to the binding payoff floor constraint is infeasible because the cutoffs $v_{t}$ given by the ODE are increasing in $t$ (see also Lemma 15). This observation, however, is not enough to prove part (ii) of Theorem 2, because there could exist a feasible solution to the auxiliary problem for which the payoff floor constraint is not binding everywhere. Therefore, in our third step, we seek conditions under which the optimal solution is given by the binding payoff floor constraint. If the payoff floor constraint must be binding at the optimal solution, then the efficient auction must be optimal whenever the solution to the binding payoff floor constraint is increasing (and hence infeasible). The sufficient condition that we identify is concavity of the monopoly profit (see Lemmas 1620 in Appendix A.3.3). We prove that for all distributions, the monopoly profit function is locally concave for valuations close to zero. Using this, we can show that the payoff floor constraint must be binding for all $t$ where $v_{t}$ is sufficiently small. This is not possible with a decreasing cutoff path if $n>\bar{N}(F)$. Hence, the efficient auction must be optimal, which proves part (ii) of Theorem 2 without imposing Assumption A4. Finally, for Proposition 4 and Theorem 3, we need Assumption A4, which requires global concavity. This implies the solution of the ODE is the optimal solution if $n<\bar{N}(F)$.

In order to establish that the payoff floor constraint must bind at the optimal solution, we construct feasible variations of solutions for which the payoff floor constraint is slack for a time interval $(a, b)$. Roughly speaking, the variation we consider spreads out the trades that happen between $a$ and $b$. For the high types in the interval $\left(v_{b}^{+}, v_{a}\right]$, we decrease the trading time, and for the low types we increase the trading time. Such a variation is always possible. We show that either some types trade in the interior of $(a, b)$, or all trades must be concentrated on the endpoint $b$. In the former case, we can move the trading times of these types towards $a$ and $b$, respectively, and for the latter, we can split the set of types that trade at $b$, delay the trading times of the low types, and advance the trading times of the high types. If the monopoly profit $v(1-F(v))$ is concave on the interval of valuations that trade between $a$ and $b$, then we prove that such a variation is not only feasible but also improves the seller's ex-ante expected profit. If $v(1-F(v))$ is convex, we have to construct a variation that concentrates the trading times of the types that trade between $a$ and $b$, rather than spreading them out. Such a variation, however, is only feasible if the trade is not already concentrated on a single point in time. Therefore, with a non-concave monopoly profit, 
we cannot rule out that the payoff floor constraint is slack on some interval if there is an atom of trade at the end of the interval 21

\section{Connecting the Mechanism Design Approach to Discrete Time Equilibria}

Our auxiliary mechanism design approach derives necessary implications from the seller's sequential rationality, which every equilibrium in discrete time must satisfy, and formulates them as the payoff floor constraint which is imposed on the set of feasible mechanisms in continuous time. Now we establish sufficiency of the payoff floor constraint: the optimal solution to our auxiliary problem is the limit of a sequence of equilibrium outcomes in our original discrete time game. We start by showing that there exists a family of equilibria in discrete time that implements outcomes converging to the efficient auction as $\Delta \rightarrow 0$ (see Subsection 5.1). In this class of equilibria, reserve prices converge to zero as $\Delta \rightarrow 0$ and the seller's profit converges to the profit of an efficient auction. Therefore, they satisfy a generalized version of the Coase conjecture in the auction setting.

In Subsection 5.2, we construct equilibria in trigger strategies that converge to the solution of the binding payoff floor constraint as $\Delta \rightarrow 0$. On the equilibrium path, the seller uses reserve prices that approximate the reserve prices defined by the binding payoff floor constraint. The approximation is constructed such that the payoff floor constraint is strictly slack. Hence, the seller's continuation profit on the equilibrium path is above the profit of an efficient auction. A deviation by the seller is punished by switching to a Coasian equilibrium, which yields a lower profit than the seller's on-path profit. Therefore, the seller has no incentive to deviate from the equilibrium price path. In the limit as $\Delta \rightarrow 0$, the punishment profit converges to the profit of the efficient auction, and the equilibrium cutoff path converges to the solution of the binding payoff floor constraint.

Subsections 5.1 and 5.2 establish the sufficiency of the payoff floor constraint. If we denote the value of the auxiliary problem by $V_{0}$, the sufficiency result implies that $V_{0} \leq \Pi^{*}$. It remains to prove the converse $V_{0} \geq \Pi^{*}$. In words, we have to prove that there is no sequence of equilibria in which the seller can achieve a higher profit in the limit as $\Delta \rightarrow 0$, than in the optimal solution to the auxiliary problem. This is shown in Subsection 5.3. Since we have only imposed necessary conditions for equilibrium outcomes in the auxiliary problem, this result is intuitive.

\footnotetext{
${ }^{21}$ So far, we have not been able to rule out this possibility or to construct an example where a solution with this feature is optimal. Therefore, the analysis of the revenue-maximizing auctions for the non-concave case remains incomplete.
} 


\subsection{Discrete Time Approximation of the Efficient Auction}

In order to prove the existence of Coasian equilibria, we follow the approach of Ausubel and Deneckere (1989), and consider a class of symmetric equilibria in which the strategy used by the buyers has a Markov property.

Definition 1. An equilibrium $(p, b) \in \mathcal{E}(\Delta)$ is a weak-Markov equilibrium if the buyers' strategies only depend on the reserve price announced for the current period.

We first show the existence of weak-Markov equilibria. The proofs of this and the following proposition follow closely the approach of Ausubel and Deneckere (1989). Therefore, we only sketch the changes necessary to adapt their proofs to the model with multiple buyers. See Appendix B in the Supplemental Material.

Proposition 5. If the type distribution satisfies Assumption A3, then a weak-Markov equilibrium exists for every $r>0$ and $\Delta>0$.

Next, we show that weak-Markov equilibria satisfy the uniform Coase conjecture. In particular, we show that in any weak-Markov equilibrium, the seller's continuation profit conditional on $v_{t}$ converges to $\Pi^{E}\left(v_{t}\right)$ as $\Delta \rightarrow 0$ and the convergence is uniform over all $v_{t} \in[0,1]$ and all weakMarkov equilibria. In contrast to the case of one buyer analyzed by Ausubel and Deneckere (1989), the first reserve price in a continuation game where the seller's posterior is $v_{t}$ need not converge to zero as $\Delta \rightarrow 022$ Nevertheless, we obtain the Coase conjecture because prices fall arbitrarily quickly as $\Delta \rightarrow 0$. On the buyer side, the strategy is described by a cutoff for the reserve price. A buyer places a bid if and only if the current reserve price is below the cutoff. The Markov property of the buyer's strategy implies that the cutoff only depends on the buyer's type, it is independent of time and of the history of previous reserve prices. As $\Delta \rightarrow 0$, the cutoff of a buyer with type $v$ converges to the payment that this type would make in a second-price auction without reserve price. Since this cutoff is bounded away from zero, and reserve prices decline arbitrarily quickly, the delay of the allocation vanishes for all buyers as $\Delta \rightarrow 0$. Therefore, the seller's profit converges to the profit of an efficient auction. More precisely, we have the following proposition.

Proposition 6 (Uniform Coase Conjecture). Fix $r>0$. If the type distribution satisfies Assumption $\boldsymbol{A}$ 3, then for every $\varepsilon>0$, there exists $a \Delta_{\varepsilon}>0$ such that for all $\Delta<\Delta_{\varepsilon}$ and all $x \in[0,1]$ and every weak-Markov equilibrium $(p, b)$ of the game with period length $\Delta$ and distribution $F(v \mid v \leq x)$, the sellers profit $\Pi^{\Delta}(p, b \mid x)$ is bounded by $(1+\varepsilon) \Pi^{E}(x)$.

This proposition implies the following Corollary.

Corollary 2. If $n>\bar{N}(F)$ and Assumption $\boldsymbol{A} 3$ is satisfied, then the optimal solution to the auxiliary problem is the limit of a sequence of discrete time equilibria for $\Delta \rightarrow 0$.

\footnotetext{
${ }^{22}$ For the uniform distribution, this was already noted by McAfee and Vincent (1997).
} 
Moreover, uniform convergence of the seller's profit enables us to use weak-Markov equilibria as punishments in the construction of equilibria that approximate the solution to the binding payoff floor constraint.

\subsection{Discrete Time Approximation of the Solution to the Binding Payoff Floor Constraint}

In order to construct equilibria that approximate the solution to the binding payoff floor constraint, we proceed in three steps. First, we show that if the binding payoff floor constraint has a decreasing solution, then there exists a nearby solution for which the payoff floor constraint is strictly slack. In particular, we show that for each $K>1$ sufficiently small, there exists a solution with a decreasing cutoff path to the following generalized payoff floor constraint:

$$
\int_{0}^{v_{t}} e^{-r(T(x)-t)} J_{t}(x) d F_{t}^{(n)}(x)=K \Pi^{E}\left(v_{t}\right) .
$$

For $K=1$, (5.1) reduces to the original payoff floor constraint in (3.7) (divided by $F_{t}\left(v_{t}\right)$ ). Therefore, a decreasing solution that satisfies (5.1) for $K>1$ is a feasible solution to the auxiliary problem. Moreover, the slack in the original payoff floor constraint is proportional to $\Pi^{E}\left(v_{t}\right)$.

Lemma 8. Suppose $n<\bar{N}(F)$. Then there exists $\Gamma>1$ such that for all $K \in[1, \Gamma]$, there exists a feasible solution $T^{K}$ to the auxiliary problem that satisfies (5.1). For $K \searrow 1, T^{K}(v)$ converges to $T(v)$ for all $v \in[0,1]$, and the seller's expected revenue converges to the value of the auxiliary problem.

In the second step, we discretize the solution obtained in the first step so that all trades take place at times $t=0, \Delta, 2 \Delta, \ldots$. For given $K$ and $\Delta$, we define the discrete approximation $T^{K, \Delta}$ of $T^{K}$ by delaying all trades in the time interval $(k \Delta,(k+1) \Delta]$ to $(k+1) \Delta$ :

$$
T^{K, \Delta}(v):=\Delta \min \left\{k \in \mathbb{N} \mid k \Delta \geq T^{K}(v)\right\}
$$

In other words, we round up all trading times to the next integer multiple of $\Delta$. Clearly, for all $v \in[0,1]$ we have,

$$
\lim _{K \rightarrow 1} \lim _{\Delta \rightarrow 0} T^{K, \Delta}(v)=\lim _{\Delta \rightarrow 0} \lim _{K \rightarrow 1} T^{K, \Delta}(v)=T(v),
$$

and the seller's expected revenue also converges. Therefore, if we show that the functions $T^{K_{m}, \Delta_{m}}$ for some sequence $\left(K_{m}, \Delta_{m}\right)$ describe equilibrium outcomes for a sequence of equilibria $\left(p^{m}, b^{m}\right) \in$ $\mathcal{E}\left(\Delta_{m}\right)$, we have obtained the desired approximation result.

The discretization changes the continuation revenue, but we can show that the approximation loss vanishes as $\Delta$ becomes small. In particular, if $\Delta$ is sufficiently small, then the approximation 
loss is less than half of the slack in the payoff floor constraint at the solution $T^{K}$. More precisely, we have the following lemma.

Lemma 9. Suppose $n<\bar{N}(F)$. For each $K \in[1, \Gamma]$, where $\Gamma$ satisfies the condition of Lemma 8 , there exists $\bar{\Delta}_{K}^{1}$ such that for all $\Delta<\bar{\Delta}_{K}^{1}$, and all $t=0, \Delta, 2 \Delta, \ldots$,

$$
\int_{0}^{v_{t}^{K, \Delta}} e^{-r\left(T^{K, \Delta}(x)-t\right)} J_{t}(x) d F_{t}^{(n)}(x) \geq \frac{K+1}{2} \Pi^{E}\left(v_{t}\right) .
$$

This lemma shows that if $\Delta$ is sufficiently small, at each point in time $t=0, \Delta, 2 \Delta, \ldots$, the continuation payoff of the discretized solution is at least as high as $1+(K-1) / 2$ times the profit of the efficient auction.

In the final step, we show that the discretized solution $T^{K, \Delta}$ can be implemented in an equilibrium of the discrete time game. To do this, we use weak-Markov equilibria as a threat to deter any deviation from the equilibrium path by the seller. The threat is effective because the uniform Coase conjecture implies that the profit of a weak-Markov equilibrium is close to the profit of an efficient auction for any posterior along the equilibrium path. More precisely, let $\Pi^{\Delta}(p, b \mid v)$ be the continuation profit at posterior $v$ for a given equilibrium $(p, b) \in \mathcal{E}(\Delta)$ as before ${ }^{23}$ Then Proposition 6 implies that for all $K \in[1, \Gamma]$, where $\Gamma$ satisfies the condition of Lemma 8 , there exists $\bar{\Delta}_{K}^{2}$ such that, for all $\Delta<\bar{\Delta}_{K}^{2}$, there exists an equilibrium $(p, b) \in \mathcal{E}(\Delta)$ such that, for all $v \in[0,1]$,

$$
\Pi^{\Delta}(p, b \mid v) \leq \frac{K+1}{2} \Pi^{E}(v)
$$

This is the observation we will use when we invoke Proposition 6 in the following.

Now suppose we have a sequence $K_{m} \searrow 1$, where $K_{m} \in[1, \Gamma]$ as in Lemma 8 . Define $\bar{\Delta}_{K}:=$ $\max \left\{\bar{\Delta}_{K}^{1}, \bar{\Delta}_{K}^{2}\right\}$. We can construct a decreasing sequence $\Delta_{m} \searrow 0$ such that for all $m, \Delta_{m}<\bar{\Delta}_{K_{m}}$. By Lemma 9 and Proposition 6 , there exists a sequence of equilibria $\left(\hat{p}^{m}, \hat{b}^{m}\right) \in \mathcal{E}\left(\Delta_{m}\right)$ such that for all $m$ and all $t=0, \Delta_{m}, 2 \Delta_{m}, \ldots$

$$
\int_{0}^{v_{t}^{K_{m}, \Delta_{m}}} e^{-r\left(T^{K_{m}, \Delta_{m}}(x)-t\right)} J_{t}(x) d F^{(n)}(x) \geq \frac{K_{m}+1}{2} \Pi^{E}\left(v_{t}^{K_{m}, \Delta_{m}}\right) \geq \Pi\left(\hat{p}^{m}, \hat{b}^{m} \mid v_{t}^{K_{m}, \Delta_{m}}\right) .
$$

The first integral in this expression is the continuation profit at time $t$ on the candidate equilibrium path given by $T^{K_{m}, \Delta_{m}}$. This is greater or equal than the second expression by Lemma 9 . The last integral is the continuation profit at time $t$ if we switch to the punishment equilibrium. This continuation profit is smaller than the second expression by Proposition 6. Therefore, for each $m$, $\left(\hat{p}^{m}, \hat{b}^{m}\right)$ can be used to support $T^{K_{m}, \Delta_{m}}$ as an equilibrium outcome of the game indexed by $\Delta_{m}$. Denote the equilibrium that supports $T^{K_{m}, \Delta_{m}}$ by $\left(p^{m}, b^{m}\right) \in \mathcal{E}\left(\Delta_{m}\right)$. It is defined as follows: On

\footnotetext{
${ }^{23}$ If the profit differs for different histories that lead to the same posterior, we could take the supremum, but this complication does not arise with weak-Markov equilibria.
} 
the equilibrium path, the seller posts reserve prices given by $T^{K_{m}, \Delta_{m}}$ and $(3.3)$. A buyer with type $v$ bids at time $T^{K_{m}, \Delta_{m}}(v)$ as long as the seller does not deviate. By Lemma 6 , this is a best response to the seller's on-path behavior. After a deviation by the seller, she is punished by switching to the equilibrium $\left(\hat{p}^{m}, \hat{b}^{m}\right)$. Since the seller anticipates the switch to $\left(\hat{p}^{m}, \hat{b}^{m}\right)$ after a deviation, her deviation profit is bounded above by $\Pi\left(\hat{p}^{m}, \hat{b}^{m} \mid v_{t}^{K_{m}, \Delta_{m}}\right)$. Therefore, (5.3) implies that the seller does not have a profitable deviation. To summarize, we have an approximation of the solution to the binding payoff floor constraint by discrete time equilibrium outcomes.

Proposition 7. Suppose Assumption A3 is satisfied and $n<\bar{N}(F)$. Then there exists a decreasing sequence $\Delta_{m} \searrow 0$ and a sequence of equilibria $\left(p^{m}, b^{m}\right) \in \mathcal{E}\left(\Delta_{m}\right)$ such that the sequence of trading functions $T_{m}$ implemented by $\left(p^{m}, b^{m}\right)$ and the seller's ex-ante revenue $\Pi^{\Delta}\left(p^{m}, b^{m}\right)$ converge to the solution given by 4.2, and the value of the auxiliary problem, respectively.

For the case that Assumption A4 is satisfied, Proposition 7 shows that the optimal solution to the auxiliary problem is the limit of a sequence of discrete time equilibria for $\Delta \rightarrow 0$. For the case that Assumption A4 is not satisfied, we did not obtain an optimal solution to the auxiliary problem from the binding payoff floor constraint. In this case, Proposition 7 shows that a feasible solution to the auxiliary problem, which involves strictly positive reserve prices and yields a higher profit than the efficient auction, can be obtained as the limit of a sequence of discrete time equilibria for $\Delta \rightarrow 0$.

\subsection{Optimality of the Solution to the Auxiliary Problem}

Corollary 2 and Proposition 7 show that $V_{0} \leq \Pi^{*}$ for all cases where we have characterized the optimal solution of the auxiliary problem. Now we show the converse. In other words, we prove that there are no better limiting outcomes and profits than the solution and value of the auxiliary problem.

To obtain this result, we first define an $\varepsilon$-relaxed continuous time auxiliary problem. We replace the payoff floor constraint by

$$
\int_{0}^{v_{t}} e^{-r(T(x)-t)} J_{t}(v) d F_{t}^{(n)}(v) \geq \Pi^{E}\left(v_{t}\right)-\varepsilon .
$$

By the maximum theorem, the value of this problem, which we denote by $V_{\varepsilon}$, is continuous in $\varepsilon$.

Next, we formulate a discrete version of the auxiliary problem. For given $\Delta$, the feasible set of this problem is given by

$$
\begin{gathered}
T:[0,1] \rightarrow\{0, \Delta, 2 \Delta, \ldots\} \text { non-increasing } \\
\text { and } \quad \int_{0}^{v_{k \Delta}} e^{-r(T(x)-k \Delta)} J_{k \Delta}(v) d F_{k \Delta}^{(n)}(v) \geq \Pi^{E}\left(v_{k \Delta}\right) \quad \forall k \in \mathbb{N} .
\end{gathered}
$$


We denote the value of this problem by $V(\Delta)$. The second constraint requires that in each period, the seller's continuation profit on the equilibrium path exceeds the revenue from an efficient auction given the current posterior. This is a necessary condition for an equilibrium. Therefore, the seller's expected revenue in any equilibrium $(p, b) \in \mathcal{E}(\Delta)$ cannot exceed $V(\Delta)$. Moreover, for given $\varepsilon$, the feasible set of the discrete auxiliary problem is contained in the feasible set of the $\varepsilon$-relaxed continuous time auxiliary problem if $\Delta$ is sufficiently small. Formally, we have:

Lemma 10. Suppose Assumption $\boldsymbol{A} 3$ is satisfied. For all $\varepsilon>0$, there exists $\Delta_{\varepsilon}>0$ such that for all $\Delta<\Delta_{\varepsilon}$ we have

$$
\sup _{(p, b) \in \mathcal{E}(\Delta)} \Pi^{\Delta}(p, b) \leq V(\Delta) \leq V_{\varepsilon} .
$$

Since the value of the relaxed auxiliary problem is continuous in $\varepsilon$ and the left-hand side of the inequality in Lemma 10 converges to $\Pi^{*}$, we have $V_{0} \geq \Pi^{*}$ and hence we have the following proposition.

Proposition 8. Suppose Assumption A3 is satisfied. If either $n>\bar{N}(F)$, or $n<\bar{N}(F)$ and Assumption $\boldsymbol{A}_{4}$ holds, then $V_{0}=\Pi^{*}$.

\section{Concluding Remarks}

In this paper we study the role of commitment power in auctions where the seller cannot commit to future reserve prices. Our analysis draws insights from the bargaining literature, and the auction and mechanism design literature. For tractability, we make several simplifying assumptions. We conclude the paper by discussing how to relax these assumptions and future research directions.

We restrict the mechanisms in each period to be standard auctions and we focus on buyersymmetric equilibria. These assumptions ensure that the posterior beliefs at any history are truncations of the original distribution, which facilitates the analysis of the auxiliary mechanism design problem. A more general version of the auxiliary problem can be formulated to allow for more general trading rules and payment schemes. With this generalization, however, the payoff floor constraints are less tractable a priori, because the posterior beliefs at arbitrary histories lack a well-defined structure. We believe, however, that the auxiliary mechanism design approach, which overcomes the failure of revelation principle, can be applied to analyze other problems with limited commitment.

The restriction to buyer-symmetric equilibria has several consequences. It rules out the possibility that different buyers have different cutoffs for bidding at a given history. Moreover, symmetry rules out that buyers play dominated strategies in equilibrium if the seller uses second-price auctions. This latter assumption is standard. Nevertheless, it is of theoretical interest to investigate whether weakly dominated strategies can expand the payoff set of the seller. For instance, the 
second price auction has an asymmetric equilibrium in which the seller's profit is equal to zero if the reserve price is zero. Presumably, the seller would never choose a reserve price of zero if she anticipates this bidding equilibrium, but it is no longer clear that the seller can guarantee the profit of an efficient auction. Allowing for dominated strategies therefore makes the characterization of the worst punishment equilibrium non-trivial. An understanding of the worst punishment, however, is crucial for the formulation of the payoff floor constraint. In particular, a punishment equilibrium that reduces the seller's profit to zero would allow us to construct an equilibrium in trigger strategies in which the seller can get the full commitment profit. The possibility of a zero-profit equilibrium in the auction setting seems unlikely, but we have not conducted an analysis that allows for asymmetric and weakly dominated strategies and therefore leave this as a future exercise.

Our way of modeling limited commitment assumes that the seller can commit to the terms of trade within a single period, no matter how small the period length is. This assumption is consistent with the durable goods monopoly literature - the prime aim of which is to analyze the role of commitment power in the bilateral trade environment. We believe this modeling approach to limited commitment is intuitive, and the assumption is also realistic in many auction practices.

Another way to formulate the problem of non-commitment is to allow renegotiation. This approach, which has been investigated in the dynamic contracting literature, is concerned with continuing relationships between a principal and an agent. If the two parties trade repeatedly, any private information revealed by the agent will be fully exploited by the principal in future interactions. This leads to the familiar "ratchet effect" (see Freixas, Guesnerie, and Tirole, 1985). The ratchet effect can be mitigated if the principal and the agent can enter a long-term contract that protects the agent from exploitation even if the contract can be renegotiated (see Hart and Tirole, 1988; Dewatripont, 1989; Laffont and Tirole, 1990). Our model differs from this literature because the sequential auction game ends with a sale as soon as some buyer actively reveals some private information by placing a bid. Therefore, there is no ratchet effect. Moreover, in a model with multiple bidders, modeling renegotiation introduces new conceptual issues, such as the protocol of multiple-person bargaining and signaling in the renegotiation phase.

In the present paper, we take a step towards understanding the role of commitment power in auctions. Our aim is to provide a deeper conceptual understanding of the economics behind commitment, and to provide a useful methodology to handle limited-commitment problems. We therefore chose the classic single-object environment initiated by Myerson (1981). In applications, one might be interested in multiple-unit auctions as well as auctions with entry of new buyers. These issues are interesting and of practical relevance. We believe our framework and methodology will be useful to address these questions.

Dynamic auctions with limited commitment also open a whole set of new theoretical issues. Many questions that have been studied for auctions with full commitment have their counterparts in our framework with limited commitment. In particular, one interesting set of questions is how 
various market design details matter under limited commitment. For instance, one could consider the role of secret reserve prices, where the auctioneer openly solicits bids without publicly announcing a reserve price. In this environment, one would further ask whether the auctioneer can ever commit to her privately set reserve prices. A more theoretical direction is to explore information disclosure in auctions. A monopolistic seller, even though she lacks commitment power, has control over information flows generated by the mechanism. Will this information be used strategically to the seller's interest? Would the ability to withhold information enforce or weaken the commitment power? We leave these questions to future research.

\section{A Omitted Proofs}

\section{A.1 Proofs for Section 2}

Proof of Lemma 2. In the main paper we slightly abuse notation by using $p_{t}$ both for the seller's (possibly mixed) strategy and the announced reserve price at a given history. This should not lead to confusion in the main part but for this proof we make a formal distinction. We denote the reserve price announced in period $t$ by $x_{t}$. A history is therefore given by $h_{t}=\left(x_{0}, \ldots, x_{t-\Delta}\right)$. Furthermore we denote by $h_{t^{+}}=\left(h_{t}, x_{t}\right)=\left(x_{0}, \ldots, x_{t-\Delta}, x_{t}\right)$ a history in which the reserve prices $x_{0}, \ldots, x_{t-\Delta}$ have been announced in periods $t=0, \ldots, t-\Delta$ but no buyer has bid in these periods, and the seller has announced $x_{t}$ in period $t$, but buyers have not yet decided whether they bid or not. For any two histories $h_{t}=\left(x_{0}, x_{\Delta}, \ldots, x_{t-\Delta}\right)$ and $h_{s}^{\prime}=\left(x_{0}^{\prime}, x_{\Delta}^{\prime}, \ldots, x_{s-\Delta}^{\prime}\right)$, with $s \leq t$, we define a new history

$$
h_{t} \oplus h_{s}^{\prime}=\left(x_{0}^{\prime}, x_{\Delta}^{\prime}, \ldots, x_{s-\Delta}^{\prime}, x_{s}, \ldots, x_{t-\Delta}\right) .
$$

That is, $h_{t} \oplus h_{s}^{\prime}$ is obtained by replacing the initial period $s$ subhistory in $h_{t}$ with $h_{s}^{\prime}$. Finally, we can similarly define $h_{t^{+}} \oplus h_{s}^{\prime}$ for $s<t$. With this notation we can state the proof of the lemma.

Consider any equilibrium $(p, b) \in \mathcal{E}(\Delta)$ in which the seller randomizes on the equilibrium path. The idea of the proof is that we can inductively replace randomization on the equilibrium path by a deterministic reserve price and at the same time weakly increase the sellers ex-ante revenue. We first construct an equilibrium $\left(p^{0}, b^{0}\right) \in \mathcal{E}(\Delta)$ in which the seller earns the same expected profit as in $(p, b)$, but does not randomize at $t=0$. If the seller uses a pure action at $t=0$, we can set $\left(p^{0}, b^{0}\right)=(p, b)$. Otherwise, if the seller randomizes over several prices at $t=0$, she must be indifferent between all prices in the support of $p_{0}\left(h_{0}\right)$. Therefore, we can define $p_{0}^{0}\left(h_{0}\right)$ as the distribution that puts probability one on a single price $x_{0} \in \operatorname{supp} p_{0}\left(h_{0}\right)$. If we leave the seller's strategy unchanged for all other histories $\left(p_{t}^{0}\left(h_{t}\right)=p_{t}\left(h_{t}\right)\right.$, for all $t>0$ and all $\left.h_{t} \in H_{t}\right)$ and set $b^{0}=b$, we have defined an equilibrium $\left(p^{0}, b^{0}\right)$ that gives the seller the same payoff as $(p, b)$ and specifies a pure action for the seller at $t=0$.

Next we proceed inductively. Suppose we have already constructed an equilibrium $\left(p^{m}, b^{m}\right)$ in 
which the seller does not randomize on the equilibrium path up to $t=m \Delta$, but uses a mixed action on the equilibrium path at $(m+1) \Delta$. We want to construct an equilibrium $\left(p^{m+1}, b^{m+1}\right)$ with a pure action for the seller on the equilibrium path at $(m+1) \Delta$. Suppose that in the equilibrium equilibrium $\left(p^{m}, b^{m}\right)$, the highest type in the posterior at $(m+1) \Delta$ is some type $\beta_{(m+1) \Delta}^{0}>0$. We select a price in the support of the seller's mixed action at $(m+1) \Delta$, which we denote by $x_{(m+1) \Delta}^{0}$, such that the expected payoff of $\beta_{(m+1) \Delta}^{0}$ at $h_{t^{+}}=\left(h_{t}, x_{(m+1) \Delta}^{0}\right)$ is weakly smaller than the expected payoff at $h_{t}$. In other words, we pick a price that is (weakly) bad news for the buyer with type $\beta_{(m+1) \Delta}^{0}$. This will be the equilibrium price announced in period $t=(m+1) \Delta$ in the equilibrium $\left(p^{m+1}, b^{m+1}\right)$. The formal construction of the equilibrium is rather complicated. The rough idea is that, first we posit that after $x_{(m+1) \Delta}^{0}$ was announced in period $(m+1) \Delta,\left(p^{m+1}, b^{m+1}\right)$ prescribes the same continuation as $\left(p^{m}, b^{m}\right)$. Second, on the equilibrium path up to period $m \Delta$, we change the reserve prices such that the same marginal types as before are indifferent between buying immediately and waiting in all periods $t=0, \ldots, m \Delta$. Since we have chosen $x_{(m+1) \Delta}^{0}$ to be bad news, this leads to (weakly) higher prices for $t=0, \ldots, m \Delta$, and therefore we can show that the seller's expected profit increases weakly. Finally, we have to specify what happens after a deviation from the equilibrium path by the seller in periods $t=0, \ldots,(m+1) \Delta$. Consider the onequilibrium history $h_{t}$ in period $t$ for $\left(p^{m+1}, b^{m+1}\right)$. We identify a history $\hat{h}_{t}$ for which the posterior in the original equilibrium $(p, b)$ is the same posterior as at $h_{t}$ in the new equilibrium. If at $h_{t}$, the seller deviates from $p^{m+1}$ by announcing the reserve price $\hat{x}_{t}$, than we define $\left(p^{m+1}, b^{m+1}\right)$ after $h_{t^{+}}=\left(h_{t}, \hat{x}_{t}\right)$ using the strategy prescribed by $(p, b)$ for the subgame starting at $\hat{h}_{t^{+}}=\left(\hat{h}_{t^{+}}, \hat{x}_{t}\right)$. We will show that with this definition, the seller does not have an incentive to deviate.

Next, we formally construct the sequence of equilibria $\left(p^{m}, b^{m}\right), m=1,2, \ldots$, and show that this sequence converges to an equilibrium $\left(p^{\infty}, b^{\infty}\right)$ in which the seller never randomizes on the equilibrium path and achieves an expected revenue at least as high as the expected revenue in $(p, b)$. We first identify a particular equilibrium path of $\left(p^{0}, b^{0}\right)$ with a sequence of reserve prices $h_{\infty}^{0}=\left(x_{0}^{0}, x_{\Delta}^{0}, \ldots\right)$ and the corresponding buyer cutoffs $\beta^{0}=\left(\beta_{0}^{0}, \beta_{\Delta}^{0}, \ldots\right)$ that specify the seller's posteriors along the path $h_{\infty}^{0}=\left(x_{0}^{0}, x_{\Delta}^{0}, \ldots\right){ }^{24}$ Then we construct an equilibrium $\left(p^{m}, b^{m}\right)$ such that the following properties hold: for $t=0, \ldots, m \Delta$, the equilibrium prices $x_{t}^{m}$ chosen by the seller are weakly higher than $x_{t}^{0}$ and the equilibrium cutoffs $\beta_{t}^{m}$ are exactly $\beta_{t}^{0}$; for $t>m \Delta$, or off the equilibrium path, the strategies coincide with what $\left(p^{0}, b^{0}\right)$ prescribes at some properly identified histories, so that the two strategy profiles prescribe the same continuation payoffs at their respective histories.

In order to determine $h_{\infty}^{0}=\left(x_{0}^{0}, x_{\Delta}^{0}, \ldots\right)$ and $\beta^{0}=\left(\beta_{0}^{0}, \beta_{\Delta}^{0}, \ldots\right)$ we start at $t=0$ and define $x_{0}^{0}$ as the seller's pure action in period zero in the equilibrium $\left(p^{0}, b^{0}\right)$ and set $\beta_{0}^{0}=1$. Next we proceed inductively. Suppose we have fixed $x_{t}^{0}$ and $\beta_{t}^{0}$ for $t=0, \Delta, \ldots$ To define $x_{t+\Delta}^{0}$, we select a price

\footnotetext{
${ }^{24}$ Note that the cutoffs $\beta_{t}^{0}$ are the equilibrium cutoffs which may be different from the cutoffs that would arise if the seller used pure actions with prices $x_{0}^{0}, x_{\Delta}^{0}, \ldots$ on the equilibrium path.
} 
in the support of the seller's mixed action at history $h_{t+\Delta}^{0}=\left(x_{0}^{0}, \ldots, x_{t}^{0}\right)$ in the equilibrium $\left(p^{0}, b^{0}\right)$ such that the expected payoff of the cutoff buyer type $\beta_{t}^{0}$, conditional on $x_{t+\Delta}^{0}$ is announces, is no larger than this type's expected payoff at the beginning of period $t+\Delta$ before a reserve price is announces ${ }^{25}$ We then pick $\beta_{t+\Delta}^{0}$ as the cutoff buyer type following history $\left(x_{0}^{0}, \ldots, x_{t}^{0}, x_{t+\Delta}^{0}\right)$.

$\left(p^{0}, b^{0}\right)$ was already defined. We proceed inductively and construct equilibrium $\left(p^{m+1}, b^{m+1}\right)$ for $m=0,1, \ldots$ as follows.

(1) On the equilibrium path at $t=(m+1) \Delta$, the seller plays a pure action and announces the reserve price $x_{(m+1) \Delta}^{m+1}:=x_{(m+1) \Delta}^{0}$.

(2) On the equilibrium path at $t=0, \Delta, \ldots, m \Delta$, the seller's pure action $x_{t}^{m+1}$ is chosen such that the buyers' on-path cutoff types in periods $t=\Delta, \ldots,(m+1) \Delta$ is $\beta_{t}^{m+1}=\beta_{t}^{0}$, where $\beta_{t}^{0}$ was defined above.

(3) On the equilibrium path at the history $h_{t^{+}}=\left(x_{0}, \ldots, x_{t}\right)$ for $t=0, \Delta,(m+1) \Delta$, each buyer bids if and only if $v^{i} \geq \beta_{t}^{m+1}=\beta_{t}^{0}$.

(4) at $t>(m+1) \Delta$ : for any history $h_{t}=\left(x_{0}, \ldots, x_{t-\Delta}\right)$ in which no deviation has occurred at or before $(m+1) \Delta$, the seller's (mixed) action is $p^{m+1}\left(h_{t}\right):=p^{0}\left(h_{t} \oplus\left(x_{0}^{0}, \ldots, x_{(m+1) \Delta}^{0}\right)\right)$. For any history $h_{t^{+}}=\left(x_{0}, \ldots, x_{t-\Delta}, x_{t}\right)$ in which no deviation has occurred at or before $(m+1) \Delta$, the buyer's strategy is defined by $b^{m+1}\left(h_{t^{+}}\right):=b^{0}\left(h_{t^{+}} \oplus\left(x_{0}^{0}, \ldots, x_{(m+1) \Delta}^{0}\right)\right)$.

(5) For any off-path history $h_{t}=\left(x_{0}, \ldots, x_{t-\Delta}\right)$ in which the seller's first deviation from the equilibrium path occurs at $s \leq(m+1) \Delta$, the seller's (mixed) action is prescribed by $p^{m+1}\left(h_{t}\right):=$ $p^{0}\left(h_{t} \oplus\left(x_{0}^{0}, \ldots, x_{s-\Delta}^{0}\right)\right)$. For any off-path history $h_{t^{+}}=\left(x_{0}, \ldots, x_{t-\Delta}, x_{t}\right)$ in which the seller's first deviation from the equilibrium path occurs in period $s \leq(m+1) \Delta$, the buyer's strategy is $b^{m+1}\left(h_{t^{+}}\right):=b^{0}\left(h_{t^{+}} \oplus\left(x_{0}^{0}, \ldots, x_{s-\Delta}^{0}\right)\right)$.

In this definition, (1) and (2) define the seller's pure actions on the equilibrium path up to $(m+1) \Delta$. The prices defined in (1) and (2) are chosen such that bidding according to the cutoffs $\beta_{t}^{m+1}$ is optimal for the buyers. Part (4) defines the equilibrium strategies for all remaining on-path histories and after deviations that occur in periods after $(m+1) \Delta$, that is, in periods where the seller can still mix on the equilibrium path. The equilibrium proceeds as in $\left(p^{0}, b^{0}\right)$ at the history where the seller used the prices $x_{0}^{0}, \ldots, x_{(m+1) \Delta}^{0}$ in the first $m+1$ periods. This ensures that the continuation strategy profile is taken from the continuation of an on-path history of the equilibrium $\left(p^{0}, b^{0}\right)$, where the seller's posterior in period $(m+1) \Delta$ is the same as in the equilibrium $\left(p^{m+1}, b^{m+1}\right)$. Finally, (5) defines the continuation after a deviation by the seller at a period in which we have

\footnotetext{
${ }^{25}$ If the seller plays a pure action at $h_{t+\Delta}^{0}$, than $x_{t+\Delta}^{0}$ the price prescribed with probability one by the pure action. If the seller randomizes at $h_{t+\Delta}^{0}$, there must be one realization, which, together with the continuation following it, gives the buyer a payoff weakly smaller than the average.
} 
already defined a pure action. If the seller deviates at a history $h_{t}=\left(x_{0}^{m}, \ldots, x_{s-\Delta}^{m}\right)$, then we use the continuation strategy of $\left(p^{0}, b^{0}\right)$, at the history $\left(x_{0}^{0}, \ldots, x_{s-\Delta}^{0}\right)$.

We proceed by proving a series of claims showing that we have indeed constructed an equilibrium.

Claim 1. The expected payoff of the cutoff buyer $\beta_{(m+1) \Delta}^{m}=\beta_{(m+1) \Delta}^{0}$ at the on-path history $h_{(m+1) \Delta}^{m}=\left(x_{0}^{m}, \ldots, x_{m \Delta}^{m}\right)$ in the candidate equilibrium $\left(p^{m}, b^{m}\right)$ is the same as its payoff at the on-path history $h_{(m+1) \Delta}^{0}=\left(x_{0}^{0}, \ldots, x_{m \Delta}^{0}\right)$ in the candidate equilibrium $\left(p^{0}, b^{0}\right)$.

Proof. This follows immediately from (1)-(3) above.

Claim 2. The expected payoff of the cutoff buyer $\beta_{(m+1) \Delta}^{m+1}=\beta_{(m+1) \Delta}^{0}$ at the on-path history $h_{((m+1) \Delta)^{+}}^{m+1}=\left(x_{0}^{m+1}, \ldots, x_{m \Delta}^{m+1}, x_{(m+1) \Delta}^{m+1}\right)$ in the candidate equilibrium $\left(x^{m+1}, x^{m+1}\right)$ is the same as this cutoff type's expected payoff at the on-path history $h_{((m+1) \Delta)^{+}}^{0}=\left(x_{0}^{0}, \ldots, x_{m \Delta}^{0}, x_{(m+1) \Delta}^{0}\right)$ in the candidate equilibrium $\left(p^{0}, b^{0}\right)$.

Proof. By construction, $x_{(m+1) \Delta}^{m+1}=x_{(m+1) \Delta}^{0}$. It follows from part (4) that $\left(p^{m+1}, b^{m+1}\right)$ and $\left(p^{0}, b^{0}\right)$ are identical on the equilibrium path from period $(m+2) \Delta$ onwards. The claim follows.

Claim 3. The expected payoff of the cutoff buyer $\beta_{(m+1) \Delta}^{m+1}=\beta_{(m+1) \Delta}^{0}$ at the on-path history $h_{(m+1) \Delta}^{m+1}=\left(x_{0}^{m+1}, \ldots, x_{m \Delta}^{m+1}\right)$ in the candidate equilibrium $\left(p^{m+1}, b^{m+1}\right)$ is weakly lower than this cutoff type's expected payoff at the on-path history $h_{(m+1) \Delta}^{0}=\left(x_{0}^{0}, \ldots, x_{m \Delta}^{0}\right)$ in the equilibrium $\left(p^{0}, b^{0}\right)$.

Proof. In the candidate equilibrium $\left(p^{m+1}, b^{m+1}\right)$, the cutoff type's payoffs at histories $h_{(m+1) \Delta}^{m+1}$ and $h_{((m+1) \Delta)^{+}}^{m+1}$ are the same because the seller plays a pure action in period $(m+1) \Delta$. In the equilibrium $\left(p^{0}, b^{0}\right)$, the cutoff type's payoff at history $h_{((m+1) \Delta)^{+}}^{m+1}$ is weakly lower than his payoff at history $h_{(m+1) \Delta}^{0}$ because of the definition of $x_{(m+1) \Delta}^{0}$ (which chosen to give the cutoff type a lower expected payoff than the expected payoff at $\left.h_{(m+1) \Delta}^{0}\right)$. The claim then follows from Claim 2 .

Claim 4. The expected payoff of the cutoff buyer $\beta_{(m+1) \Delta}^{m+1}=\beta_{(m+1) \Delta}^{0}$ at the on-path history $h_{(m+1) \Delta}^{m+1}=\left(x_{0}^{m+1}, \ldots, x_{m \Delta}^{m+1}\right)$ in the candidate equilibrium $\left(p^{m+1}, b^{m+1}\right)$ is weakly lower than this cutoff type's expected payoff at the on-path history $h_{(m+1) \Delta}^{m}=\left(x_{0}^{m}, \ldots, x_{m \Delta}^{m}\right)$ in the candidate equilibrium $\left(p^{m}, b^{m}\right)$.

Proof. By Claim 1, the cutoff type's expected payoff at the on-path history $h_{(m+1) \Delta}^{m}=\left(x_{0}^{m}, \ldots, x_{m \Delta}^{m}\right)$ in the candidate equilibrium $\left(p^{m}, b^{m}\right)$ is the same as its payoff at the on-path history $h_{(m+1) \Delta}^{0}=$ $\left(x_{0}^{0}, \ldots, x_{m \Delta}^{0}\right)$ in the candidate equilibrium $\left(p^{0}, b^{0}\right)$. The claim then follows from Claim 3.

Claim 5. For each $m=0,1, \ldots$ and $t=0,1, \ldots, m \Delta$, we have $x_{t}^{m+1} \geq x_{t}^{m}$. 
Proof. By Claim 4, the cutoff type $\beta_{(m+1) \Delta}^{m+1}=\beta_{(m+1) \Delta}^{m}=\beta_{(m+1) \Delta}^{0}$ in period $(m+1) \Delta$ on the equilibrium path in the candidate equilibrium $\left(p^{m+1}, b^{m+1}\right)$ has a weakly lower payoff than its expected payoff in the candidate equilibrium $\left(p^{m}, b^{m}\right)$. To keep this cutoff indifferent in period $m \Delta$ in both candidate equilibria, we must have $x_{m \Delta}^{m+1} \geq x_{m \Delta}^{m}$. Then to keep the cutoff type $\beta_{m \Delta}^{m+1}=$ $\beta_{m \Delta}^{m}=\beta_{m \Delta}^{0}$ indifferent in period $(m-1) \Delta$, we must have $x_{(m-1) \Delta}^{m+1} \geq x_{(m-1) \Delta}^{m}$. The proof is then completed by induction.

Claim 6. The seller's (time 0) expected payoff in the candidate equilibrium $\left(p^{m+1}, b^{m+1}\right)$ is weakly higher than the seller's expected payoff in the equilibrium $\left(p^{0}, b^{0}\right)$.

Proof. By parts (1)-(3) of the construction, at $t=0, \ldots, m \Delta,\left(p^{m+1}, b^{m+1}\right)$ and $\left(p^{m}, b^{m}\right)$ have the same buyer cutoffs on the equilibrium path. At $t=(m+1) \Delta$, the seller in $\left(p^{m+1}, b^{m+1}\right)$ chooses $x_{(m+1) \Delta}^{m+1}$ that is in the support of the seller's strategy in $\left(p^{m}, b^{m}\right)$ in that period (note that even though we haven't show that $\left(p^{m}, b^{m}\right)$ is an equilibrium, the seller is indeed indifferent in $\left(p^{m}, b^{m}\right)$ at $(m+1) \Delta$ because the play switch to $\left(p^{0}, b^{0}\right)$ with identical continuation payoffs by Part $(4)$ of the construction). It then follows from Claim 5 that the seller's (time 0) expected payoff in $\left(p^{m+1}, b^{m+1}\right)$ is weakly higher than the seller's (time 0$)$ expected payoff in $\left(p^{m}, b^{m}\right)$. The claim is proved by repeating this argument.

Claim 7. For $t=\Delta, \ldots,(m+1) \Delta$, the seller's expected payoff at the on-path history $\left(x_{0}^{m+1}, \ldots, x_{t-\Delta}^{m+1}\right)$, in the candidate equilibrium $\left(p^{m+1}, b^{m+1}\right)$ is weakly higher than the seller's expected at the history $\left(x_{0}^{0}, \ldots, x_{t-\Delta}^{0}\right)$ in equilibrium $\left(p^{0}, b^{0}\right)$.

Proof. Denote $m_{t}=t / \Delta$ so that $t=m_{t} \Delta$ and consider $\left(p^{m_{t}}, b^{m_{t}}\right)$. By parts (1)-(3) of the construction, the buyer's cutoff type at $\left(x_{0}^{m_{t}}, \ldots, x_{t-\Delta}^{m_{t}}\right)$ in this equilibrium is the same as the buyer's cutoff type at $\left(x_{0}^{0}, \ldots, x_{t-\Delta}^{0}\right)$ in equilibrium $\left(p^{0}, b^{0}\right)$. By part (4) of the construction, the seller's payoff at history $\left(x_{0}^{m_{t}}, \ldots, x_{t-\Delta}^{m_{t}}\right)$ in $\left(p^{m_{t}}, b^{m_{t}}\right)$ coincides with the seller's payoff at history $\left(x_{0}^{0}, \ldots, x_{t-\Delta}^{0}\right)$ in equilibrium $\left(p^{0}, b^{0}\right)$. Now consider the candidate equilibrium $\left(p^{m_{t}+1}, b^{m_{t}+1}\right)$ and the history $\left(x_{0}^{m_{t}+1}, \ldots, x_{t-\Delta}^{m_{t}+1}\right)$. By claim $5,\left(x_{0}^{m_{t}+1}, \ldots, x_{t-\Delta}^{m_{t}+1}\right) \geq\left(x_{0}^{m_{t}}, \ldots, x_{t-\Delta}^{m_{t}}\right)$. Note that the candidate equilibrium $\left(p^{m_{t}+1}, b^{m_{t}+1}\right)$ further differs from the equilibrium $\left(p^{m_{t}}, b^{m_{t}}\right)$ on the equilibrium path in period $t+\Delta$. But $x_{t}^{m_{t}+1}$ is in the support of the seller's randomization in $\left(p^{m_{t}}, b^{m_{t}}\right)$ (which makes the seller indifferent by part (4) of the equilibrium construction — see the proof in Claim 6). Therefore, the seller's payoff at $\left(x_{0}^{m_{t}+1}, \ldots, x_{t-\Delta}^{m_{t}+1}\right)$ in the equilibrium $\left(p^{m_{t}+1}, b^{m_{t}+1}\right)$ is weakly greater than at $\left(x_{0}^{m_{t}}, \ldots, x_{t-\Delta}^{m_{t}}\right)$ in the equilibrium $\left(p^{m_{t}+1}, b^{m_{t}+1}\right)$. This completes the proof of the claim.

Claim 8. For each $m=0,1, \ldots,\left(p^{m+1}, b^{m+1}\right)$ such constructed is indeed an equilibrium.

Proof. The buyer's optimality condition follows immediately from the construction. Now consider the seller. By part (5) of the construction, for any off-path history $h_{t}=\left(x_{0}, \ldots, x_{t-\Delta}\right)$ in which the seller's first deviation from the equilibrium path occurs at $s \leq(m+1) \Delta$, the continuation strategy 
profile prescribed by $\left(p^{m+1}, b^{m+1}\right)$ is exactly that prescribed by $\left(p^{0}, b^{0}\right)$ at a corresponding history $h_{t} \oplus\left(x_{0}^{0}, \ldots, x_{s-\Delta}^{0}\right)$ with exactly the same expected payoff (the payoff is the same due to the fact that the seller's strategies coincide and the fact that the buyer's cutoff at $h_{t}$ in $\left(p^{m+1}, b^{m+1}\right)$ is the same as that at $h_{t} \oplus\left(x_{0}^{0}, \ldots, x_{s-\Delta}^{0}\right)$ in $\left.\left(p^{0}, b^{0}\right)\right)$. Hence there is no profitable deviation at $h_{t}$ in $\left(p^{m+1}, b^{m+1}\right)$ just as there is no profitable deviation at $h_{t} \oplus\left(x_{0}^{0}, \ldots, x_{s-\Delta}^{0}\right)$ in $\left(p^{0}, b^{0}\right)$.

By part (4) of the construction, at $t>(m+1) \Delta$, for any history $h_{t}=\left(x_{0}, \ldots, x_{t-\Delta}\right)$ in which no deviation has occurred at or before $(m+1) \Delta$, the seller's strategy at $h_{t}$ in $\left(p^{m+1}, b^{m+1}\right)$ coincides with the seller's strategy at $h_{t} \oplus\left(x_{0}^{m}, \ldots, x_{(m+1) \Delta}^{m}\right)$, with exactly the same continuation payoffs (see the previous paragraph). Hence there is no profitable deviation at $h_{t}$ in $\left(p^{m+1}, b^{m+1}\right)$.

Now consider parts (1)-(3) of the construction, for $t=0, \ldots,(m+1) \Delta$. By Claim 6 and 7 , staying on the equilibrium path gives the seller a weakly higher payoff than that from the equilibrium $\left(p^{0}, b^{0}\right)$ at the corresponding history. But deviation from the equilibrium path triggers a switch to $\left(p^{0}, b^{0}\right)$ at a corresponding history. Since there is no deviation in $\left(p^{0}, b^{0}\right)$, deviation becomes even less desirable in $\left(p^{m+1}, b^{m+1}\right)$. This completes the proof of the claim.

So far, we have obtained a sequence of equilibria $\left\{\left(p^{m}, b^{m}\right)\right\}_{m=0}^{\infty}$. Denote the limit of this sequence by $\left(p^{\infty}, b^{\infty}\right)$. It is easy to check that the limit is well-defined. It remains to show that $\left(p^{\infty}, b^{\infty}\right)$ is an equilibrium. It is clear that buyers do not have an incentive to deviate. For the seller, suppose the seller has a profitable deviation at some history $h_{m \Delta}$. By the definition of $\left(p^{\infty}, b^{\infty}\right)$ and the construction of the sequence $\left\{\left(p^{m}, b^{m}\right)\right\}_{m=0}^{\infty}$, the continuation play at $h_{t}$ in the candidate equilibrium $\left(p^{\infty}, b^{\infty}\right)$, where $h_{t}$ is a history with $h_{m \Delta}$ as its subhistory, will coincide with continuation play at $h_{t}$ prescribed by equilibrium $\left(p^{m^{\prime}}, b^{m^{\prime}}\right)$ for any $m^{\prime} \geq m$, which is in turn described by $p^{0}\left(h_{t} \oplus\left(x_{0}^{0}, \ldots, x_{(m-1) \Delta}^{0}\right)\right)$ and $b^{0}\left(h_{t^{+}} \oplus\left(x_{0}^{0}, \ldots, x_{(m-1) \Delta}^{0}\right)\right)$ by part (5) of the equilibrium construction. Since $\left(p^{m^{\prime}}, b^{m^{\prime}}\right)$ is an equilibrium, this particular deviation is not profitable in the equilibrium $\left(p^{m^{\prime}}, b^{m^{\prime}}\right)$ for any $m^{\prime} \geq m$. But the on-path payoff of $\left(p^{m^{\prime}}, b^{m^{\prime}}\right)$ converges to that of $\left(p^{\infty}, b^{\infty}\right)$, and we have just argued that the payoff after this particular deviation is the same for both $\left(p^{m^{\prime}}, b^{m^{\prime}}\right)$ and $\left(p^{\infty}, b^{\infty}\right)$. This contradicts the assumption of profitable deviation.

Proof of Lemma 3. Fix a history $h_{t}$. Note that if all buyers bid, then by the standard argument, it is optimal for each bidder to bid their true values. Therefore, it is sufficient to show that each buyer will submit a bid. By Lemma 1, we only need to show $\beta_{t}\left(h_{t}, p_{t}\right)=0$. Suppose by contradiction that $\beta_{t}\left(h_{t}, p_{t}\right)>0$. Consider a positive type $\beta_{t}\left(h_{t}, p_{t}\right)-\varepsilon$, where $\varepsilon>0$. By Lemma 1 , if this type follows the equilibrium strategy and waits, he wins only if his opponents all have types lower than $\beta_{t}\left(h_{t}, p_{t}\right)-\varepsilon$, and he can only win in period $t+\Delta$ or later at a price no smaller than 0 . If he deviates and bids his true value in period $t$, it follows from Lemma 1 that he wins in period $t$ at a price 0 if all of his opponents have types lower than $\beta_{t}\left(h_{t}, p_{t}\right)$. Therefore, the deviation is strictly profitable for type $\beta_{t}\left(h_{t}, p_{t}\right)-\varepsilon$, contradicting the definition of $\beta_{t}\left(h_{t}, p_{t}\right)$. 


\section{A.2 Proofs for Section 3}

Proof of Lemma 4. By Restrictions 1 and 4, the discounted trading time of buyer $i$ only depends on $v^{i}$ and $v^{(n)}$. If $v^{i}<v^{(n)}$ we have $q^{i}\left(v^{i}, v^{-i}\right)=0$. Otherwise, Restriction 3 implies that $e^{-r \tau^{i}\left(v^{i}, v^{-i}\right)}=e^{-r T\left(v^{(n)}\right)}$ for some function $T$ which is independent of $i$ by symmetry (Restriction 2). This observation, together with Restriction 1 and the random tie-breaking rule, implies that if $v^{i}=v^{(n)}$,

$$
q^{i}\left(v^{i}, v^{-i}\right)=\frac{e^{-r T\left(v^{i}\right)}}{\#\left\{j: v^{j}=v^{(n)}\right\}} .
$$

Restriction 5 implies that $T$ is non-increasing.

Proof of Lemma 5. It is sufficient to show that we can find a payment rule $\pi$, together with $\tau$ as defined in the Lemma, is incentive compatible. First, $\tau^{i}\left(v^{i}, v^{-i}\right)=\mathbf{1}_{\left\{v^{i}=v^{(n)}\right\}} T\left(v^{i}\right)$, implies that the interim discounted allocation probability $Q^{i}\left(v^{i}\right)=F\left(v^{i}\right)^{n-1} e^{-r T\left(v^{i}\right)}$, which is non-decreasing if $T$ is non-increasing. Next, we define the instantaneous payment rule $\pi^{i}$ by

$$
\pi^{i}\left(v^{i}, v^{-i}\right)=\frac{M^{i}\left(v^{i}\right)}{Q^{i}\left(v^{i}\right)}
$$

where $M^{i}\left(v^{i}\right)$ is given by the payoff equivalence formula:

$$
M^{i}\left(v^{i}\right)=v^{i} Q^{i}\left(v^{i}\right)-\int_{0}^{v^{i}} Q^{i}(x) d x
$$

The discounted payment rule $m^{i}$ for bidder $i$ is specified as

$$
m^{i}\left(v^{i}, v^{-i}\right)=\frac{\mathbf{1}_{\left\{v^{i}=v^{(n)}\right\}} e^{-r T\left(v^{i}\right)}}{\#\left\{j: v^{j}=v^{(n)}\right\}} \frac{M^{i}\left(v^{i}\right)}{Q^{i}\left(v^{i}\right)}=q^{i}\left(v^{i}, v^{-i}\right) \frac{M^{i}\left(v^{i}\right)}{Q^{i}\left(v^{i}\right)} .
$$

Therefore,

$$
E_{v^{-i}}\left[m^{i}\left(v^{i}, v^{-i}\right)\right]=E_{v^{-i}}\left[q^{i}\left(v^{i}, v^{-i}\right)\right] \frac{M^{i}\left(v^{i}\right)}{Q^{i}\left(v^{i}\right)}=M^{i}\left(v^{i}\right)
$$

By construction, $M^{i}\left(v^{i}\right)$ satisfies the payoff equivalence formula and $Q^{i}\left(v^{i}\right)$ is monotone, so $\left(M^{i}, Q^{i}\right)$ is incentive compatible, and thus $(\tau, \pi)$ is incentive compatible.

Proof of Lemma 6. Consider first periods $t$ when at least one type trades, that is, $t \in T([0,1])$. If $p_{t}$ is the price that a buyer who trades at time $t$ has to pay, then we have

$$
\begin{aligned}
Q^{i}\left(v_{t}^{+}\right) & =F\left(v_{t}^{+}\right)^{n-1} e^{-r T\left(v_{t}^{+}\right)} \\
\text {and } \quad U^{i}\left(v_{t}^{+}\right) & =F\left(v_{t}^{+}\right)^{n-1} e^{-r T\left(v_{t}^{+}\right)}\left(v_{t}^{+}-p_{t}\right) .
\end{aligned}
$$


Inserting this into the payoff equivalence formula, we obtain

$$
\left(F\left(v_{t}^{+}\right)\right)^{n-1} e^{-r T\left(v_{t}^{+}\right)}\left(v_{t}^{+}-p_{t}\right)=\int_{0}^{v_{t}^{+}} e^{-r T(v)}(F(v))^{n-1} d v,
$$

which can be rearranged to $[3.3)$. For $t \notin T([0,1])$, we can set $p_{t}=p_{\underline{t}}$ where $\underline{t}=\inf \{s \mid(s, t] \cap T=\emptyset\}$ is the latest time $s$ before $t$ for which we have already defined $p_{s}$. Since $v_{t}^{+}$is constant on $[\underline{t}, t]$ this yields (3.3) again.

Proof of Lemma匇. The IC constraint at time 0 requires that, for any $v^{i}$ and $\hat{v}^{i}$,

$$
\begin{aligned}
& E_{v^{-i}}\left[q^{i}\left(v^{i}, v^{-i}\right)\right] v^{i}-E_{v^{-i}}\left[q^{i}\left(v^{i}, v^{-i}\right) \pi^{i}\left(v^{i}, v^{-i}\right)\right] \\
\geq & E_{v^{-i}}\left[q^{i}\left(\hat{v}^{i}, v^{-i}\right)\right] v^{i}-E_{v^{-i}}\left[q^{i}\left(\hat{v}^{i}, v^{-i}\right) \pi^{i}\left(\hat{v}^{i}, v^{-i}\right)\right] .
\end{aligned}
$$

We want to argue it implies IC constraints for any future period $t>0$. To see this, consider $v^{i}$ and $\hat{v}^{i}$ with $v^{i} \leq v_{t}$ and $\hat{v}^{i} \leq v_{t}$. Note that, if $v^{(n)}>v_{t}$, then $q^{i}\left(v^{i}, v^{-i}\right)=q^{i}\left(\hat{v}^{i}, v^{-i}\right)=0$. Therefore,

$$
\begin{aligned}
& E_{v^{-i}}\left[q^{i}\left(v^{i}, v^{-i}\right)\right] v^{i}-E_{v^{-i}}\left[q^{i}\left(v^{i}, v^{-i}\right) \pi^{i}\left(v^{i}, v^{-i}\right)\right] \\
= & \left\{E_{v^{-i}}\left[q^{i}\left(v^{i}, v^{-i}\right) \mid v^{(n)} \leq v_{t}\right] v^{i}-E_{v^{-i}}\left[q^{i}\left(v^{i}, v^{-i}\right) \pi^{i}\left(v^{i}, v^{-i}\right) \mid v^{(n)} \leq v_{t}\right]\right\} \operatorname{Pr}\left(v^{(n)} \leq v_{t}\right),
\end{aligned}
$$

and an analogous equation holds for $\hat{v}^{i}$. Therefore, the IC constraint at time 0 implies (3.6).

Proof of Theorem 1. Suppose by contradiction that, $T$ is feasible but $T(v)=\infty$ for some $v>0$. Since $T$ is non-increasing, there exists $w \in(0,1)$ such that $T(v)=\infty$ for all $v \in[0, w)$ and $T(v)<\infty$ for all $v \in(w, 1]$. The left-hand side of the payoff floor constraint can be rewritten as, for all $t<\infty$,

$$
\int_{w}^{v_{t}} e^{-r(T(x)-t)} J_{t}(x) d F^{(n)}(x)
$$

Since $T(v)<\infty$ for all $v \in(w, 1]$, we have $v_{t} \rightarrow w$ as $t \rightarrow \infty$. Hence, as $t \rightarrow \infty$, the limit of the left-hand side is zero:

$$
\lim _{t \rightarrow \infty} \int_{0}^{v_{t}} e^{-r(T(x)-t)} J_{t}(x) d F^{(n)}(x)=\lim _{t \rightarrow \infty} \int_{w}^{v_{t}} e^{-r(T(x)-t)} J_{t}(x) d F^{(n)}(x)=0 .
$$

The limit of right-hand side of the payoff floor constraint as $t \rightarrow \infty$, however, is strictly positive:

$$
\lim _{t \rightarrow \infty} \int_{0}^{v_{t}} J_{t}(x) d F^{(n)}(x)=\int_{0}^{w}\left(x-\frac{F(w)-F(x)}{f(x)}\right) d F^{(n)}(x)>0 .
$$

Therefore, the payoff floor constraint must be violated for sufficiently large $t$, which contradicts the feasibility of $T$.

Proof of Proposition 1. Let $\delta(v):=e^{-r T(v)}$ denote the discount factor for type $v$ who trades at time 
$T(v)$. We can rewrite the auxiliary problem as a maximization problem with $\delta(v)$ as the choice variable:

$$
\begin{gathered}
\sup _{\delta} \int_{0}^{1} \delta(v) J(v) f^{(n)}(v) d v \\
\text { s.t. } \delta(v) \in[0,1], \text { and non-decreasing, } \\
\forall v \in[0,1]: \int_{0}^{v} \delta(s) J(s \mid s \leq v) f^{(n)}(s) d s \geq \delta\left(v^{+}\right) \int_{0}^{v} J(s \mid s \leq v) f^{(n)}(s) d s .
\end{gathered}
$$

Let $\bar{\pi}$ be the supremum of this maximization problem and let $\left(\delta_{k}\right)$ be a sequence of feasible solutions of this problem such that

$$
\lim _{k \rightarrow \infty} \int_{0}^{1} \delta_{k}(v) J(v) f^{(n)}(v) d v=\bar{\pi} .
$$

By Helly's selection theorem, there is a subsequence $\left(\delta_{k_{\ell}}\right)$, and a non-decreasing function $\bar{\delta}:[0,1] \rightarrow$ $[0,1]$ such that $\delta_{k_{\ell}}(v) \rightarrow \bar{\delta}(v)$ for all points of continuity of $\bar{\delta}$. Hence (after selecting a subsequence), we can take $\left(\delta_{k}\right)$ to be almost everywhere convergent with a.e.-limit $\bar{\delta}$. By Lebesgue's dominated convergence theorem, we also have convergence w.r.t. the $L^{2}$-norm and hence weak convergence in $L^{2}$. Therefore

$$
\int_{0}^{1} \bar{\delta}(v) J(v) f^{(n)}(v) d v=\lim _{k \rightarrow \infty} \int_{0}^{1} \delta_{k}(v) J(v) f^{(n)}(v) d v=\bar{\pi} .
$$

It remains to show that $\bar{\delta}$ satisfies the payoff floor constraint. Suppose not. Then there exists $\hat{v} \in[0,1)$ such that

$$
\int_{0}^{\hat{v}} \bar{\delta}(s) J(s \mid s \leq \hat{v}) f^{(n)}(s) d s<\bar{\delta}\left(\hat{v}^{+}\right) \int_{0}^{\hat{v}} J(s \mid s \leq \hat{v}) f^{(n)}(s) d s .
$$

Then there also exists $v \geq \hat{v}$ such that $\bar{\delta}$ is continuous at $v$, and

$$
\int_{0}^{v} \bar{\delta}(s) J(s \mid s \leq v) f^{(n)}(s) d s<\bar{\delta}(v) \int_{0}^{v} J(s \mid s \leq v) f^{(n)}(s) d s .
$$

Define

$$
S:=\bar{\delta}(v) \int_{0}^{v} J(s \mid s \leq v) f^{(n)}(s) d s-\int_{0}^{v} \bar{\delta}(s) J(s \mid s \leq v) f^{(n)}(s) d s .
$$

Since $v$ is a point of continuity we have $\bar{\delta}(v)=\lim _{k \rightarrow \infty} \delta_{k}(v)$. Therefore, there exists $k_{v}$ such that for all $k>k_{v}$,

$$
\left|\bar{\delta}(v) \int_{0}^{v} J(s \mid s \leq v) f^{(n)}(s) d s-\delta_{k}(v) \int_{0}^{v} J(s \mid s \leq v) f^{(n)}(s) d s\right|<\frac{S}{2},
$$

and furthermore, since $\delta_{k} \rightarrow \bar{\delta}$ weakly in $L^{2}$, we can choose $k_{v}$ such for all $k>k_{v}$ also

$$
\left|\int_{0}^{v} \bar{\delta}(s) J(s \mid s \leq v) f^{(n)}(s) d s-\int_{0}^{v} \delta_{k}(s) J(s \mid s \leq v) f^{(n)}(s) d s\right|<\frac{S}{2} .
$$


Together, this implies that for all $k>k_{v}$,

$$
\int_{0}^{v} \delta_{k}(s) J(s \mid s \leq v) f^{(n)}(s) d s<\delta_{k}(v) \int_{0}^{v} J(s \mid s \leq v) f^{(n)}(s) d s,
$$

which contradicts the assumption that $\delta_{k}$ is an feasible solution of the reformulated auxiliary problem defined above.

Proof of Proposition 2. By definition, $r T=\hat{r} \hat{T}$, so the values of the objective functions under $r$ and $\hat{r}$ are the same:

$$
\int_{0}^{1} e^{-r T(x)} J(v) d F^{(n)}(x)=\int_{0}^{1} e^{-\hat{r} \hat{T}(x)} J(v) d F^{(n)}(x) .
$$

Furthermore, $\hat{T}$ is non-increasing. It remains to show that $\hat{T}$ satisfies the payoff floor under $\hat{r}$. Let $v_{t}$ and $\hat{v}_{t}$ be the cutoff paths corresponding to $T$ and $\hat{T}$, respectively. By defining $\gamma:=r / \hat{r}$, we have

$$
v_{t}=\sup \{v \mid T(v) \geq t\}=\sup \{v \mid \hat{T}(v) \geq \gamma t\}=\hat{v}_{\gamma t} .
$$

Therefore,

$$
J_{t}(v)=v-\frac{F\left(v_{t}\right)-F(v)}{f(v)}=v-\frac{F\left(\hat{v}_{\gamma t}\right)-F(v)}{f(v)}=\hat{J}_{\gamma t}(v)
$$

By inserting this into the payoff floor constraint at $t$ with $r$ and using $r T=\hat{r} \hat{T}$ again, we obtain

$$
\int_{0}^{\hat{v}_{\gamma t}} e^{-\hat{r}(\hat{T}(x)-\gamma t)} \hat{J}_{\gamma t}(v) d F^{(n)}(x) \geq \int_{0}^{\hat{v}_{\gamma t}} \hat{J}_{\gamma t}(v) d F^{(n)}(x),
$$

or equivalently,

$$
\int_{0}^{\hat{v}_{s}} e^{-\hat{r}(\hat{T}(x)-s)} \hat{J}_{s}(v) d F^{(n)}(x) \geq \int_{0}^{\hat{v}_{s}} \hat{J}_{s}(v) d F^{(n)}(x)
$$

Hence $\hat{T}$ satisfies the payoff floor constraint under $\hat{r}$ for all $s=\gamma t$.

\section{A.3 Proofs for Section 4}

Before proving Proposition 3, we first establish a lemma. We consider solutions where a strictly positive measure of types trade at the same time $t$ so that $v_{t}>v_{t}^{+}$. In other words, there is an "atom" of types that trade at $t$. The following lemma shows that if the payoff floor constraint is satisfied right after the atom, then the payoff floor constraint at $t$ (right before the atom) is strictly slack. Moreover, if we reduce the size of the atom by lowering $v_{t}$ to $v \in\left(v_{t}^{+}, v_{t}\right)$ so that some types in the atom trade earlier than $t$, the payoff floor constraint at $t$ remains strictly slack for all choices $v \in\left(v_{t}^{+}, v_{t}\right)$. This lemma is more general than needed for the proof of Proposition 3 . The extra generality will be used later. 
Lemma 11. Let $T:[0,1] \rightarrow[0,1]$ be non-increasing (not necessarily feasible) and denote the corresponding cutoff sequence by $v_{t}$. Suppose there is an "atom" at $t \geq 0$, that is, $v_{t}>v_{t}^{+}$. If the payoff floor constraint is satisfied at $t^{+}$, that is

$$
\int_{0}^{v_{t}^{+}} e^{-r(T(x)-t)}\left(x-\frac{F\left(v_{t}^{+}\right)-F(x)}{f(x)}\right) d F^{(n)}(x) \geq \int_{0}^{v_{t}^{+}}\left(x-\frac{F\left(v_{t}^{+}\right)-F(x)}{f(x)}\right) d F^{(n)}(x),
$$

then we have, for all $v \in\left(v_{t}^{+}, v_{t}\right]$,

$$
\int_{0}^{v} e^{-r(T(x)-t)}\left(x-\frac{F(v)-F(x)}{f(x)}\right) d F^{(n)}(x)>\int_{0}^{v}\left(x-\frac{F(v)-F(x)}{f(x)}\right) d F^{(n)}(x) .
$$

In particular, the payoff floor constraint is satisfied at $t$.

Proof. Fix $v \in\left(v_{t}^{+}, v_{t}\right]$. Note that the LHS of $(\mathrm{A} .2)$ can be rewritten as

$$
\begin{aligned}
& \int_{0}^{v} e^{-r(T(x)-t)}\left(x-\frac{F(v)-F(x)}{f(x)}\right) d F^{(n)}(x) \\
= & \int_{v_{t}^{+}}^{v}\left(x-\frac{F(v)-F(x)}{f(x)}\right) d F^{(n)}(x)+\int_{0}^{v_{t}^{+}} e^{-r(T(x)-t)}\left(x-\frac{F\left(v_{t}^{+}\right)-F(x)}{f(x)}\right) d F^{(n)}(x) \\
& -\int_{0}^{v_{t}^{+}} e^{-r(T(x)-t)}\left(\frac{F(v)-F\left(v_{t}^{+}\right)}{f(x)}\right) d F^{(n)}(x) \\
\geq & \int_{v_{t}^{+}}^{v}\left(x-\frac{F(v)-F(x)}{f(x)}\right) d F^{(n)}(x)+\int_{0}^{v_{t}^{+}}\left(x-\frac{F\left(v_{t}^{+}\right)-F(x)}{f(x)}\right) d F^{(n)}(x) \\
& -\int_{0}^{v_{t}^{+}} e^{-r(T(x)-t)}\left(\frac{F(v)-F\left(v_{t}^{+}\right)}{f(x)}\right) d F^{(n)}(x),
\end{aligned}
$$

where the equality follows because all types in $\left(v_{t}^{+}, v\right]$ trade at time $t$, and the inequality follows from A.1. Similarly, the RHS of A.2 can be rewritten as

$\int_{v_{t}^{+}}^{v}\left(x-\frac{F(v)-F(x)}{f(x)}\right) d F^{(n)}(x)+\int_{0}^{v_{t}^{+}}\left(x-\frac{F\left(v_{t}^{+}\right)-F(x)}{f(x)}\right) d F^{(n)}(x)-\int_{0}^{v_{t}^{+}}\left(\frac{F(v)-F\left(v_{t}^{+}\right)}{f(x)}\right) d F^{(n)}(x)$.

Hence, A.2 holds if

$$
-\int_{0}^{v_{t}^{+}} e^{-r(T(x)-t)}\left(\frac{F(v)-F\left(v_{t}^{+}\right)}{f(x)}\right) d F^{(n)}(x)>-\int_{0}^{v_{t}^{+}}\left(\frac{F(v)-F\left(v_{t}^{+}\right)}{f(x)}\right) d F^{(n)}(x)
$$

or equivalently

$$
\int_{0}^{v_{t}^{+}}\left(1-e^{-r(T(x)-t)}\right)\left(\frac{F(v)-F\left(v_{t}^{+}\right)}{f(x)}\right) d F^{(n)}(x)>0 .
$$

Since $T(x)>t$ for $x<v_{t}^{+}$and $F(v)-F\left(v_{t}^{+}\right)>0$ for $v>v_{t}^{+}$, the last inequality holds and the proof is complete. 
Proof of Proposition 3. The "if" part is trivial. For the "only if" part, suppose there is another feasible solution $\tilde{T}$ other than the efficient auction $T \equiv 0$. Let $\tilde{v}_{t}$ denote the cutoff path corresponding to $\tilde{T}$. Note first that the range of $\tilde{T}$ cannot be a singleton because this would imply that $\tilde{T}(v)=t$ for all $v \in[0,1]$ for some $t>0$. Then the expected revenue would be given by

$$
e^{-r t} \int_{0}^{1} J(v) d F^{(n)}(v)
$$

which is strictly lower than the revenue from an efficient auction at time 0 . Therefore, the payoff floor constraint is violated at $t=0$, contradicting the feasibility of $\tilde{T}$. Hence, we can assume that there exists some time $s$ such that $\tilde{T}(v)<s$ for all $v>\tilde{v}_{s}$ and $\tilde{T}(v)>s$ for all $v<\tilde{v}_{s}$. Then we can define a new feasible solution

$$
\hat{T}(v):= \begin{cases}0 & \text { if } v<\tilde{v}_{s} \\ \tilde{T}(v)-s & \text { if } v \geq \tilde{v}_{s}\end{cases}
$$

with corresponding cutoff path $\hat{v}_{t}$. Solution $\hat{T}$ is feasible because $\tilde{T}$ satisfies the payoff floor constraint for all $t \geq s$. Moreover, we must have $0<\hat{v}_{0}^{+}<1$. We can invoke Lemma 11 by setting $t=0$ and $v=v_{0}=1$ to obtain

$$
\int_{0}^{1} e^{-r \hat{T}(x)} J(x) d F^{(n)}(x)>\int_{0}^{1} J(x) d F^{(n)}(x) .
$$

The left hand side of the above inequality is the revenue from $\hat{T}$, while the right hand side is the revenue from $T \equiv 0$. This completes the proof.

Proof of Remark 1. We first show that every distribution with well-defined $\phi$ must have $\phi \geq-1$. Suppose by contradiction that $\phi<-1$. Then there exists $\varepsilon>0$ such that, for all $v \in(0, \varepsilon]$, $f^{\prime}(v) v / f(v) \leq-1$. We divide both sides by $v$ and integrate to obtain, for $0<x<\varepsilon$,

$$
\int_{x}^{\varepsilon} \frac{f^{\prime}(v)}{f(v)} d v \leq-\int_{x}^{\varepsilon} \frac{1}{v} d v
$$

which is equivalent to $f(x) \geq f(\varepsilon) \varepsilon / x$. But this implies that the distribution function $F$ is not well-defined because, for $z>0$,

$$
F(z)=\int_{0}^{z} f(x) d x \geq f(\varepsilon) \varepsilon \int_{0}^{z} \frac{1}{x} d x=\infty .
$$

Therefore, we must have $\phi \geq-1$. The same argument can used to show that $\phi \neq-1$ if $f^{\prime}(v) v / f(v)$ is independent of $v$ for all $v \in(0, \varepsilon]$ for some $\varepsilon>0$.

Next we prove Theorems 2 and 3 . As outlined in the main text, their proofs are closely connected 
and several preliminary lemmas are used in both proofs. We start by considering solutions to the binding payoff floor constraint. We will show that the binding payoff floor constraint can be reduced to an ODE which will give us a candidate solution. This candidate solution, represented by a cutoff path, may not decrease over time, so it may not be feasible. The next step is to check when the candidate solution is feasible, which is then used to prove part (i) of Theorem 2. The last step shows that the payoff floor constraint must bind at the optimal solution if the cutoff $v_{t}$ is in a range where the monopoly profit $v(1-F(v))$ is concave. Since this is always true for $v$ sufficiently close to zero, we can prove part (ii) of Theorem 2 without appealing to Assumption A4. For Theorem 3. we need that the payoff floor constraint binds everywhere which is true if Assumption A4 is satisfied. Figure A.1 gives a graphical overview of the various lemmas and how they are used in the proofs of these two theorems.

\section{A.3.1 Step 1: Construct a Solution to the Binding Payoff Floor Constraint}

The (binding) payoff floor constraint we will study here will be more general than needed to prove Theorems 2 and 3 . The extra generality is important for our later analysis in Section 5 where we use equilibria of discrete time games to approximate the solution to the auxiliary problem. Our discrete approximation requires feasible solutions for which the payoff floor constraint is strictly slack, that is, for all $t \geq 0$,

$$
\int_{0}^{v_{t}} e^{-r(T(x)-t)} J_{t}(x) d F^{(n)}(x)=K \int_{0}^{v_{t}} J_{t}(x) d F^{(n)}(x),
$$

where $K \in[1, \Gamma]$ with some $\Gamma>0$. We will refer constraint A.3 as the generalized (binding) payoff floor constraint. Note that our earlier binding payoff floor constraint is a special case with $K=1$. The following lemma shows that the generalized payoff floor constraint A.3) can be reduced to an ODE. For $K=1$, this ODE reduces to (4.1).

We assume for now that the solutions $T$ and $v_{t}$ for which the generalized payoff floor constraint is binding are continuously differentiable. We will show later in Lemma 13 that this differentiability property holds for every solution for which the payoff floor is binding.

Lemma 12. Suppose $T(x)$ satisfies A.3 for all $t \in(a, b)$ and suppose $T$ is continuously differentiable with $-\infty<T^{\prime}(v)<0$ for all $v \in\left(v_{b}, v_{a}\right)$ and $v_{t}$ is continuously differentiable for all $t \in(a, b)$. Then $v_{t}$ is twice continuously differentiable on $(a, b)$ and is characterized by

$$
\frac{\ddot{v}_{t}}{\dot{v}_{t}}+g\left(v_{t}, K\right) \dot{v}_{t}+h\left(v_{t}, K\right)\left(\dot{v}_{t}\right)^{2}+r=0
$$

where

$$
g\left(v_{t}, K\right)=\frac{f^{\prime}\left(v_{t}\right)}{f\left(v_{t}\right)}-\frac{\left\{\left(2-\frac{1}{K}\right) v_{t} F^{n-1}\left(v_{t}\right)-2 \int_{0}^{v_{t}} F^{n-1}(v) d v\right\} f\left(v_{t}\right)}{(n-1) \int_{0}^{v_{t}}\left[F\left(v_{t}\right)-F(v)\right] F^{n-2}(v) f(v) v d v}
$$




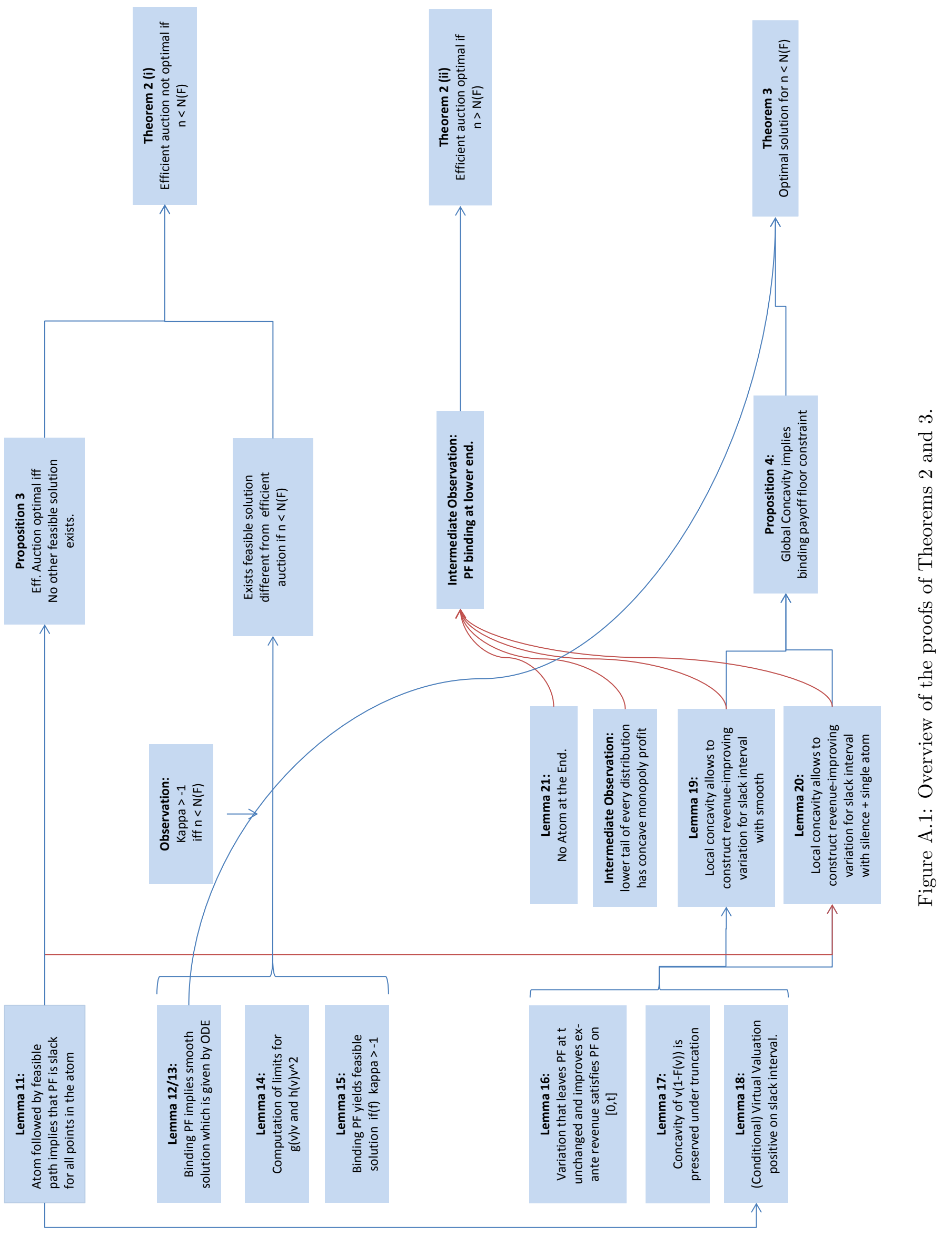


and

$$
h\left(v_{t}, K\right)=\frac{K-1}{r K} \frac{F^{n-2}\left(v_{t}\right) f^{2}\left(v_{t}\right) v_{t}}{\int_{0}^{v_{t}}\left[F\left(v_{t}\right)-F(v)\right] F^{n-2}(v) f(v) v d v} .
$$

Proof. We first rewrite $\mathrm{A} .3$ as

$$
\int_{0}^{v_{t}} e^{-r T(x)} J_{t}(x) d F^{(n)}(x)=K e^{-r t} \int_{0}^{v_{t}} J_{t}(x) d F^{(n)}(x)
$$

Since $v_{t}$ is continuously differentiable on $(a, b)$, we can differentiate A.3 on both sides to obtain

$$
\begin{aligned}
& e^{-r t} v_{t} f^{(n)}\left(v_{t}\right) \dot{v}_{t}-\int_{0}^{v_{t}} e^{-r T(x)} \frac{f\left(v_{t}\right) \dot{v}_{t}}{f(x)} d F^{(n)}(x) \\
= & -K r e^{-r t} \int_{0}^{v_{t}} J_{t}(x) d F^{(n)}(x)+K e^{-r t} v_{t} f^{(n)}\left(v_{t}\right) \dot{v}_{t}-K e^{-r t} \int_{0}^{v_{t}} \frac{f\left(v_{t}\right) \dot{v}_{t}}{f(x)} d F^{(n)}(x),
\end{aligned}
$$

where we have used $\frac{\partial J_{t}(x)}{\partial t}=-\frac{f\left(v_{t}\right) \dot{v}_{t}}{f(x)}$. This equation can be further simplified

$$
\begin{aligned}
& -\int_{0}^{v_{t}} e^{-r T(x)} \frac{f\left(v_{t}\right) \dot{v}_{t}}{f(x)} d F^{(n)}(x) \\
= & -K r e^{-r t} \int_{0}^{v_{t}} J_{t}(x) d F^{(n)}(x)+(K-1) e^{-r t} f^{(n)}\left(v_{t}\right) v_{t} \dot{v}_{t}-K e^{-r t} \int_{0}^{v_{t}} \frac{f\left(v_{t}\right) \dot{v}_{t}}{f(x)} d F^{(n)}(x) .
\end{aligned}
$$

Since $T$ is continuous and has a bounded derivative, $\dot{v}_{t}>0$. By assumption, $f\left(v_{t}\right)>0$, so we can divide the previous equation by $-f\left(v_{t}\right) \dot{v}_{t}$ to obtain

$$
\begin{aligned}
& \int_{0}^{v_{t}} e^{-r T(x)} \frac{1}{f(x)} d F^{(n)}(x) \\
= & K \frac{r e^{-r t}}{f\left(v_{t}\right) \dot{v}_{t}} \int_{0}^{v_{t}} J_{t}(x) d F^{(n)}(x)+K e^{-r t} \int_{0}^{v_{t}} \frac{1}{f(x)} d F^{(n)}(x)-(K-1) e^{-r t} \frac{f^{(n)}\left(v_{t}\right)}{f\left(v_{t}\right)} v_{t} .
\end{aligned}
$$

This equation, together with our assumption that $f(v)$ is continuously differentiable, implies that $v_{t}$ is twice continuously differentiable. Hence, we may differentiate on both sides.

$$
\begin{aligned}
& \frac{d}{d t}\left(r e^{-r t} \frac{\int_{0}^{v_{t}} J_{t}(x) f^{(n)}(x) d x}{f\left(v_{t}\right) \dot{v}_{t}}\right) \\
= & -r^{2} e^{-r t} \frac{\int_{0}^{v_{t}} J_{t}(x) f^{(n)}(x) d x}{f\left(v_{t}\right) \dot{v}_{t}} \\
& +r e^{-r t}\left(\frac{v_{t} f^{(n)}\left(v_{t}\right) \dot{v}_{t}+\dot{v}_{t} \int_{0}^{v_{t}}\left(-\frac{f\left(v_{t}\right)}{f(x)}\right) f^{(n)}(x) d x}{f\left(v_{t}\right) \dot{v}_{t}}-\frac{\left(f^{\prime}\left(v_{t}\right)\left(\dot{v}_{t}\right)^{2}+f\left(v_{t}\right) \ddot{v}_{t}\right) \int_{0}^{v_{t}} J_{t}(x) f^{(n)}(x) d x}{\left(f\left(v_{t}\right) \dot{v}_{t}\right)^{2}}\right) \\
= & r e^{-r t}\left(\frac{v_{t} f^{(n)}\left(v_{t}\right) \dot{v}_{t}-f\left(v_{t}\right) \int_{0}^{v_{t}} \frac{f^{(n)}(x)}{f(x)} d x \dot{v}_{t}}{f\left(v_{t}\right) \dot{v}_{t}}-\frac{\left(\dot{v}_{t} \frac{f^{\prime}\left(v_{t}\right)}{f\left(v_{t}\right)}+\frac{\ddot{v}_{t}}{\dot{v}_{t}}+r\right) \int_{0}^{v_{t}} J_{t}(x) f^{(n)}(x) d x}{f\left(v_{t}\right) \dot{v}_{t}}\right),
\end{aligned}
$$


where we have used $\frac{\partial^{2} J_{t}(x)}{\partial t^{2}}=-\frac{f^{\prime}\left(v_{t}\right)\left(\dot{v}_{t}\right)^{2}+f\left(v_{t}\right) \ddot{v}_{t}}{f(x)}$. Next, note that

$$
\begin{aligned}
\frac{d}{d t}\left(e^{-r t} \int_{0}^{v_{t}} \frac{1}{f(x)} d F^{(n)}(x)\right) & =-r e^{-r t} \int_{0}^{v_{t}} \frac{1}{f(x)} d F^{(n)}(x)+e^{-r t} \frac{f^{(n)}\left(v_{t}\right)}{f\left(v_{t}\right)} \dot{v}_{t} \\
& =-r e^{-r t} \int_{0}^{v_{t}} \frac{1}{f(x)} d F^{(n)}(x)+e^{-r t} n F^{n-1}\left(v_{t}\right) \dot{v}_{t}
\end{aligned}
$$

and

$$
\begin{aligned}
\frac{d}{d t}\left(e^{-r t} \frac{f^{(n)}\left(v_{t}\right)}{f\left(v_{t}\right)} v_{t}\right) & =\frac{d}{d t}\left(e^{-r t} n F^{n-1}\left(v_{t}\right) v_{t}\right) \\
& =-r e^{-r t} n F^{n-1}\left(v_{t}\right) v_{t}+e^{-r t} n(n-1) F^{n-2}\left(v_{t}\right) f\left(v_{t}\right) v_{t} \dot{v}_{t}+e^{-r t} n F^{n-1}\left(v_{t}\right) \dot{v}_{t} .
\end{aligned}
$$

Therefore, differentiating (A.4) on both sides yields

$$
\begin{aligned}
& e^{-r t} n F^{n-1}\left(v_{t}\right) \dot{v}_{t} \\
= & K r e^{-r t}\left(\frac{v_{t} f^{(n)}\left(v_{t}\right) \dot{v}_{t}-f\left(v_{t}\right) \int_{0}^{v_{t}} \frac{f^{(n)}(x)}{f(x)} d x \dot{v}_{t}}{f\left(v_{t}\right) \dot{v}_{t}}-\frac{\left(\dot{v}_{t} \frac{f^{\prime}\left(v_{t}\right)}{f\left(v_{t}\right)}+\frac{\ddot{v}_{t}}{\dot{v}_{t}}+r\right) \int_{0}^{v_{t}} J_{t}(x) f^{(n)}(x) d x}{f\left(v_{t}\right) \dot{v}_{t}}\right) \\
& -K r e^{-r t} \int_{0}^{v_{t}} \frac{1}{f(x)} d F^{(n)}(x)+K e^{-r t} n F^{n-1}\left(v_{t}\right) \dot{v}_{t} \\
& +(K-1) r e^{-r t} n F^{n-1}\left(v_{t}\right) v_{t}-(K-1) e^{-r t} n(n-1) F^{n-2}\left(v_{t}\right) f\left(v_{t}\right) v_{t} \dot{v}_{t}-(K-1) e^{-r t} n F^{n-1}\left(v_{t}\right) \dot{v}_{t} .
\end{aligned}
$$

This can be simplified into

$$
\begin{aligned}
0= & K r\left(\frac{v_{t} f^{(n)}\left(v_{t}\right) \dot{v}_{t}-f\left(v_{t}\right) \int_{0}^{v_{t}} \frac{f^{(n)}(x)}{f(x)} d v \dot{v}_{t}}{f\left(v_{t}\right) \dot{v}_{t}}-\frac{\left(\dot{v}_{t} \frac{f^{\prime}\left(v_{t}\right)}{f\left(v_{t}\right)}+\frac{\ddot{v}_{t}}{\dot{v}_{t}}+r\right) \int_{0}^{v_{t}} J_{t}(x) f^{(n)}(x) d x}{f\left(v_{t}\right) \dot{v}_{t}}\right) \\
& -K r \int_{0}^{v_{t}} \frac{1}{f(x)} d F^{(n)}(x)+(K-1) r n F^{n-1}\left(v_{t}\right) v_{t}-(K-1) n(n-1) F^{n-2}\left(v_{t}\right) f\left(v_{t}\right) v_{t} \dot{v}_{t} .
\end{aligned}
$$

Multiplying both sides by $f\left(v_{t}\right) \dot{v}_{t}$, we obtain

$$
\begin{aligned}
0= & K r v_{t} f^{(n)}\left(v_{t}\right) \dot{v}_{t}-K r \int_{0}^{v_{t}} \frac{f^{(n)}(x)}{f(x)} d x f\left(v_{t}\right) \dot{v}_{t}-K r\left(\dot{v}_{t} \frac{f^{\prime}\left(v_{t}\right)}{f\left(v_{t}\right)}+\frac{\ddot{v}_{t}}{\dot{v}_{t}}+r\right) \int_{0}^{v_{t}} J_{t}(x) f^{(n)}(x) d x, \\
& -K r \int_{0}^{v_{t}} \frac{1}{f(x)} d F^{(n)}(x) f\left(v_{t}\right) \dot{v}_{t} \\
& +(K-1) r n F^{n-1}\left(v_{t}\right) f\left(v_{t}\right) \dot{v}_{t} v_{t}-(K-1) n(n-1) F^{n-2}\left(v_{t}\right)\left(f\left(v_{t}\right)\right)^{2} v_{t}\left(\dot{v}_{t}\right)^{2} .
\end{aligned}
$$

Collecting terms we obtain

$$
K r\left(\dot{v}_{t} \frac{f^{\prime}\left(v_{t}\right)}{f\left(v_{t}\right)}+\frac{\ddot{v}_{t}}{\dot{v}_{t}}+r\right) \int_{0}^{v_{t}} J_{t}(x) f^{(n)}(x) d x=(2 K-1) r v_{t} f^{(n)}\left(v_{t}\right) \dot{v}_{t}-2 K r \int_{0}^{v_{t}} \frac{f^{(n)}(x)}{f(x)} d x f\left(v_{t}\right) \dot{v}_{t}
$$




$$
-(K-1) n(n-1) F^{n-2}\left(v_{t}\right)\left(f\left(v_{t}\right)\right)^{2} v_{t}\left(\dot{v}_{t}\right)^{2} .
$$

Hence we have

$$
\begin{gathered}
\frac{\ddot{v}_{t}}{\dot{v}_{t}}+\underbrace{\left(\frac{f^{\prime}\left(v_{t}\right)}{f\left(v_{t}\right)}-\frac{\frac{(2 K-1)}{K} f^{(n)}\left(v_{t}\right) v_{t}-2 f\left(v_{t}\right) n \int_{0}^{v_{t}} F^{n-1}(x) d x}{\int_{0}^{v_{t}} J_{t}(x) f^{(n)}(x) d x}\right)}_{=: g\left(v_{t}, K\right)} \dot{v}_{t} \\
+\underbrace{\frac{(K-1)}{r K} \frac{n(n-1) F^{n-2}\left(v_{t}\right)\left(f\left(v_{t}\right)\right)^{2} v_{t}}{v_{0}^{v_{t}} J_{t}(x) f^{(n)}(x) d x}}_{=: h\left(v_{t}, K\right)}\left(\dot{v}_{t}\right)^{2}+r=0 .
\end{gathered}
$$

Using

$$
\begin{aligned}
\int_{0}^{v_{t}} J_{t}(x) f^{(n)}(x) d x= & n \int_{0}^{v_{t}}\left(x-\frac{F\left(v_{t}\right)-F(x)}{f(x)}\right) F^{n-1}(x) f(x) d x \\
= & n \int_{0}^{v_{t}}\left(x F^{n-1}(x) f(x)-F\left(v_{t}\right) F^{n-1}(x)+F^{n}(x)\right) d x \\
= & n \int_{0}^{v_{t}} F^{n-1}(x) f(x) x d x-n F\left(v_{t}\right) \int_{0}^{v_{t}} F^{n-1}(x) d x+n \int_{0}^{v_{t}} F^{n}(x) d x \\
= & n \int_{0}^{v_{t}} F^{n-1}(x) f(x) x d x-n F\left(v_{t}\right) F^{n-1}\left(v_{t}\right) v_{t}+n(n-1) F\left(v_{t}\right) \int_{0}^{v_{t}} F^{n-2}(x) f(x) x d x \\
& +n F^{n}\left(v_{t}\right) v_{t}-n \int_{0}^{v_{t}} n F^{n-1}(x) f(x) x d x \\
= & -(n-1) n \int_{0}^{v_{t}} F^{n-1}(x) f(x) x d x+n(n-1) F\left(v_{t}\right) \int_{0}^{v_{t}} F^{n-2}(x) f(x) x d x \\
= & (n-1) n \int_{0}^{v_{t}}\left(F\left(v_{t}\right)-F(x)\right) F^{n-2}(x) f(x) x d x
\end{aligned}
$$

we have

$$
g\left(v_{t}, K\right)=\frac{f^{\prime}\left(v_{t}\right)}{f\left(v_{t}\right)}-\frac{\left\{\left(2-\frac{1}{K}\right) v_{t} F^{n-1}\left(v_{t}\right)-2 \int_{0}^{v_{t}} F^{n-1}(v) d v\right\} f\left(v_{t}\right)}{(n-1) \int_{0}^{v_{t}}\left[F\left(v_{t}\right)-F(v)\right] F^{n-2}(v) f(v) v d v}
$$

and

$$
h\left(v_{t}, K\right)=\frac{K-1}{r K} \frac{F^{n-2}\left(v_{t}\right) f^{2}\left(v_{t}\right) v_{t}}{\int_{0}^{v_{t}}\left[F\left(v_{t}\right)-F(v)\right] F^{n-2}(v) f(v) v d v} .
$$

Next we show that, if the payoff floor is binding for $T$ and $v_{t}$, then they must be continuously differentiable. Therefore, the differentiability assumption in Lemma 12 is not necessary. This result will only be used for the original binding payoff floor constraint, for which we want to show that the solution to the ODE is the only solution to the binding payoff floor constraint. For the generalized payoff floor constraint we will not need a uniqueness result.

Lemma 13. Let $T$ be a feasible solution for which (3.7) holds with equality for all $t>0$. Then 
(i) $T$ is strictly decreasing for $v \in\left[0, v_{0}^{+}\right]$.

(ii) $T$ is continuously differentiable with $T^{\prime}(v)<0$ for all $v \in\left(0, v_{0}^{+}\right)$.

(iii) $v_{t}$ is twice continuously differentiable for all $t>0$ where $v_{t}>0$.

Proof. Note that part (i) and part (ii) imply that $v_{t}$ is continuously differentiable for all $t>0$ where $v_{t}>0$. Part (iii) then follows from Lemma 12 .

To prove (i) and (ii), we proceed in several steps. First, we show that $T$ is strictly decreasing except for a possible interval $\left[0, v_{a}\right]$ with $a<\infty$, where $T$ is constant and equal to $a$. Suppose not. Then there exists a trading time $s$ such that $T^{-1}(s)=\left(w, v_{s}\right]$ where $w<v_{s}$. Since the payoff floor constraint is binding for all $t>0$, we also have

$$
\int_{0}^{v_{t}^{+}} e^{-r(T(x)-t)}\left(x-\frac{F\left(v_{t}^{+}\right)-F(x)}{f(x)}\right) d F^{(n)}(x)=\int_{0}^{v_{t}^{+}}\left(x-\frac{F\left(v_{t}^{+}\right)-F(x)}{f(x)}\right) d F^{(n)}(x),
$$

for all $t>0$. This implies

$$
\begin{aligned}
& \int_{0}^{v_{s}}\left(x-\frac{F\left(v_{s}\right)-F(x)}{f(x)}\right) d F^{(n)}(x)-\int_{0}^{w}\left(x-\frac{F(w)-F(x)}{f(x)}\right) d F^{(n)}(x) \\
= & \int_{0}^{v_{s}} e^{-r(T(x)-s)}\left(x-\frac{F\left(v_{s}\right)-F(x)}{f(x)}\right) d F^{(n)}(x)-\int_{0}^{w} e^{-r(T(x)-s)}\left(x-\frac{F(w)-F(x)}{f(x)}\right) d F^{(n)}(x) \\
= & \int_{w}^{v_{s}} J_{s}(x) d F^{(n)}(x)+\left(F(w)-F\left(v_{s}\right)\right) \int_{0}^{w} e^{-r(T(x)-s)} \frac{1}{f(x)} d F^{(n)}(x) .
\end{aligned}
$$

On the other hand we have

$$
\begin{aligned}
& \int_{0}^{v_{s}}\left(x-\frac{F\left(v_{s}\right)-F(x)}{f(x)}\right) d F^{(n)}(x)-\int_{0}^{w}\left(x-\frac{F(w)-F(x)}{f(x)}\right) d F^{(n)}(x) \\
= & \int_{w}^{v_{s}} J_{s}(x) d F^{(n)}(x)+\left(F(w)-F\left(v_{s}\right)\right) \int_{0}^{w} \frac{1}{f(x)} d F^{(n)}(x) .
\end{aligned}
$$

Subtracting this equality from the previous one, we obtain

$$
\int_{0}^{w} e^{-r(T(x)-s)} \frac{1}{f(x)} d F^{(n)}(x)=\int_{0}^{w} \frac{1}{f(x)} d F^{(n)}(x) .
$$

This expression can only hold if $w=0$.

Next, we show that $T$ is continuous. Suppose not. Then there exists a time interval $(a, b)$ such that $v_{t}$ is positive and constant on $(a, b)$. Since $b>a$ we have

$$
\begin{array}{r}
e^{-r(b-a)} \int_{0}^{v_{b}} J_{b}(x) d F^{(n)}(x)<\int_{0}^{v_{b}} J_{b}(x) d F^{(n)}(x) \Leftrightarrow \\
e^{-r(b-a)} \int_{0}^{v_{b}} e^{-r(T(x)-b)} J_{b}(x) d F^{(n)}(x)<\int_{0}^{v_{b}} J_{b}(x) d F^{(n)}(x) \Leftrightarrow \\
\int_{0}^{v_{a}^{+}} e^{-r(T(x)-a)} J_{a}(x) d F^{(n)}(x)<\int_{0}^{v_{a}^{+}} J_{a}(x) d F^{(n)}(x) .
\end{array}
$$


where the second line follows from the binding payoff floor constraint at $b$, and the last line follows from the fact that $v_{a}^{+}=v_{b}$ and $J_{a}(x)=J_{b}(x)$. But the last line contradicts the assumption that the payoff floor constraint is satisfied at $a^{+}$. Therefore, $T$ is continuous.

Third, we show that $T$ is continuously differentiable on $\left(v_{a}, v_{0}^{+}\right)$. Since $T$ is continuous and strictly decreasing for $v \in\left(v_{a}, v_{0}^{+}\right)$, a binding payoff floor constraint for all $t \in(0, a)$ is equivalent to the condition that, for all $v \in\left(v_{a}, v_{0}^{+}\right)$,

$$
\int_{0}^{v} e^{-r T(x)}\left(x-\frac{F(v)-F(x)}{f(x)}\right) d F^{(n)}(x)=e^{-r T(v)} \int_{0}^{v}\left(x-\frac{F(v)-F(x)}{f(x)}\right) d F^{(n)}(x),
$$

which can be rearranged into

$$
e^{-r T(v)}=\frac{\int_{0}^{v} e^{-r T(x)}\left(x-\frac{F(v)-F(x)}{f(x)}\right) d F^{(n)}(x)}{\int_{0}^{v}\left(x-\frac{F(v)-F(x)}{f(x)}\right) d F^{(n)}(x)}
$$

Continuity of $T$ and continuous differentiability of $F$ imply that the left-hand side of this expression is continuously differentiable, and thus $T$ is also continuously differentiable. For the derivative we obtain

$$
\begin{aligned}
r e^{-r T(v)} T^{\prime}(v)= & \frac{e^{-r T(v)} f^{(n)}(v) v-\int_{0}^{v} e^{-r T(x)} \frac{f(v)}{f(x)} d F^{(n)}(x)}{\int_{0}^{v}\left(x-\frac{F(v)-F(x)}{f(x)}\right) d F^{(n)}(x)} \\
& -\frac{\left[f^{(n)}(v) v-\int_{0}^{v} \frac{1}{f(x)} d F^{(n)}(x)\right] \int_{0}^{v} e^{-r T(x)}\left(x-\frac{F(v)-F(x)}{f(x)}\right) d F^{(n)}(x)}{\left(\int_{0}^{v}\left(x-\frac{F(v)-F(x)}{f(x)}\right) d F^{(n)}(x)\right)^{2}} \\
\Longleftrightarrow r e^{-r T(v)} T^{\prime}(v)= & \frac{e^{-r T(v)\left[f^{(n)}(v) v-\int_{0}^{v} \frac{f(v)}{f(x)} d F^{(n)}(x)\right] \int_{0}^{v} e^{-r(T(x)-T(v))}\left(x-\frac{F(v)-F(x)}{f(x)}\right) d F^{(n)}(x)}}{\left(\int_{0}^{v}\left(x-\frac{F(v)-F(x)}{f(x)}\right) d F^{(n)}(x)\right)^{2}} \\
& -\frac{e^{-r T(v)\left[f^{(n)}(v) v-\int_{0}^{v} e^{-r(T(x)-T(v))} \frac{f(v)}{f(x)} d F^{(n)}(x)\right]}}{\int_{0}^{v}\left(x-\frac{F(v)-F(x)}{f(x)}\right) d F^{(n)}(x)} .
\end{aligned}
$$

Hence

$$
\begin{aligned}
T^{\prime}(v)=\frac{1}{r} & \frac{\left[f^{(n)}(v) v-\int_{0}^{v} \frac{f(v)}{f(x)} d F^{(n)}(x)\right] \int_{0}^{v} e^{-r(T(x)-T(v))}\left(x-\frac{F(v)-F(x)}{f(x)}\right) d F^{(n)}(x)}{\left(\int_{0}^{v}\left(x-\frac{F(v)-F(x)}{f(x)}\right) d F^{(n)}(x)\right)^{2}} \\
& -\frac{1}{r} \frac{\left[f^{(n)}(v) v-\int_{0}^{v} e^{-r(T(x)-T(v))} \frac{f(v)}{f(x)} d F^{(n)}(x)\right] \int_{0}^{v}\left(x-\frac{F(v)-F(x)}{f(x)}\right) d F^{(n)}(x)}{\left(\int_{0}^{v}\left(x-\frac{F(v)-F(x)}{f(x)}\right) d F^{(n)}(x)\right)^{2}}
\end{aligned}
$$




$$
\begin{aligned}
= & \frac{1}{r} \frac{\left[f^{(n)}(v) v-\int_{0}^{v} \frac{f(v)}{f(x)} d F^{(n)}(x)\right] \int_{0}^{v}\left(x-\frac{F(v)-F(x)}{f(x)}\right) d F^{(n)}(x)}{\left(\int_{0}^{v}\left(x-\frac{F(v)-F(x)}{f(x)}\right) d F^{(n)}(x)\right)^{2}} \\
& -\frac{1}{r} \frac{\left[f^{(n)}(v) v-\int_{0}^{v} e^{-r(T(x)-T(v))} \frac{f(v)}{f(x)} d F^{(n)}(x)\right] \int_{0}^{v}\left(x-\frac{F(v)-F(x)}{f(x)}\right) d F^{(n)}(x)}{\left(\int_{0}^{v}\left(x-\frac{F(v)-F(x)}{f(x)}\right) d F^{(n)}(x)\right)^{2}} \\
= & \frac{f(v)}{r} \frac{\left[\int_{0}^{v} e^{-r(T(x)-T(v))} \frac{1}{f(x)} d F^{(n)}(x)-\int_{0}^{v} \frac{1}{f(x)} d F^{(n)}(x)\right] \int_{0}^{v}\left(x-\frac{F(v)-F(x)}{f(x)}\right) d F^{(n)}(x)}{\left(\int_{0}^{v}\left(x-\frac{F(v)-F(x)}{f(x)}\right) d F^{(n)}(x)\right)^{2}} \\
= & \frac{f(v)}{r} \frac{\int_{0}^{v}\left(e^{-r(T(x)-T(v))}-1\right) \frac{1}{f(x)} d F^{(n)}(x)}{\int_{0}^{v}\left(x-\frac{F(v)-F(x)}{f(x)}\right) d F^{(n)}(x)} .
\end{aligned}
$$

where the second equality follows from the binding payoff floor constraint. In the last line, the numerator is strictly negative and the denominator is positive. Therefore $T^{\prime}(v)<0$. This shows part (ii) for $v \in\left(a, v_{0}^{+}\right)$.

To complete the proof for part (i) and the remaining case of part (ii), it suffices to show that $a=0$. Suppose not. Then there exists $a<\infty$ such that $T(v)=a$ for all $v \in\left[0, v_{a}\right]$. Our results obtained above imply that $v_{t}$ is continuously differentiable for $t \in(0, a)$ since $T$ is continuously differentiable, strictly decreasing, and finite on $\left(v_{a}, v_{0}^{+}\right)$. It follows from Lemma 12 that

$$
\frac{\ddot{v}_{t}}{\dot{v}_{t}}+g\left(v_{t}\right) \dot{v}_{t}+r=0
$$

Using the change of variables $y=\dot{v}_{t}$ we have

$$
y^{\prime}(v)+g(v) y(v)+r=0
$$

The solution to the differential equation is

$$
y(v)=e^{-\int_{v_{a}}^{v} g(x) d x}\left(C-\int_{v_{a}}^{v} r e^{\int_{v_{a}}^{w} g(x) d x} d w\right)
$$

where $C$ is the integration constant. Since $f$ is continuous and $f(v)>0, g(v)$ is bounded on $[\varepsilon, 1-\varepsilon]$ for every $\varepsilon>0$. Therefore, we have

$$
\lim _{v \searrow v_{a}} y(v)=C .
$$

To reach a contradiction, we differentiate the binding payoff floor constraint as in the proof of Lemma 12 to obtain

$$
\frac{r e^{-r t}}{f\left(v_{t}\right) \dot{v}_{t}} \int_{0}^{v_{t}} J_{t}(x) d F^{(n)}(x)=\int_{0}^{v_{t}} e^{-r T(x)} \frac{1}{f(x)} d F^{(n)}(x)-e^{-r t} \int_{0}^{v_{t}} \frac{1}{f(x)} d F^{(n)}(x) .
$$


Since $T(x)=t$ for $x \leq a$, the right-hand side of this expression converges to zero as $t \nearrow a$, but the left-hand side is

$$
\lim _{t \nearrow a} \frac{r e^{-r t}}{f\left(v_{t}\right) \dot{v}_{t}} \int_{0}^{v_{t}} J_{t}(x) d F^{(n)}(x)=\frac{r e^{-r a}}{f\left(v_{a}\right) C} \int_{0}^{v_{a}} J_{a}(x) d F^{(n)}(x) \neq 0
$$

This contradicts the fact that the payoff floor constraint binds at $t=a$. Hence, $a=0$.

\section{A.3.2 Step 2: Check Feasibility of the Constructed Solution}

If the ODE in (4.1) admits a decreasing solution $\left(\dot{v}_{t} \leq 0, \forall t\right)$, then the binding payoff floor constraint yields non-trivial feasible solution to the auxiliary problem. It turns out that the existence of such a solution depends on the behavior of $g(v) v$ for $v \rightarrow 0$. We denote this limit by $\kappa$. The following lemma gives an explicit expression for this constant. Again we prove a more general result that will be used in the discrete time approximation.

Lemma 14. If Assumption A2 is satisfied, we have

$$
\begin{aligned}
\kappa:=\lim _{v \rightarrow 0} g(v) v & =\phi-\frac{((n-1) \phi+n-2)(n \phi+n+1)}{(n-1)(1+\phi)}, \\
\lim _{v \rightarrow 0} g(v, K) v & =\kappa-\frac{K-1}{K}\left(n \phi+n+2+\frac{\phi+2}{(n-1)(1+\phi)}\right),
\end{aligned}
$$

and

$$
\lim _{v \rightarrow 0} h(v, K) v^{2}=\frac{1}{r} \frac{K-1}{K}(n+\phi n+1)(n+\phi n-\phi) .
$$

Proof. We define the following functions:

$$
\begin{aligned}
X(v) & :=\frac{F^{n-1}(v) f(v) v}{(n-1) \int_{0}^{v}[F(v)-F(s)] F^{n-2}(s) f(s) s d s}, \\
Y(v) & :=\frac{2 f(v) \int_{0}^{v} F^{n-1}(s) d s}{(n-1) \int_{0}^{v}[F(v)-F(s)] F^{n-2}(s) f(s) s d s} .
\end{aligned}
$$

With these definitions we have

$$
g(v)=g(v, 1)=\frac{f^{\prime}(v)}{f(v)}-X(v)+Y(v)
$$

and

$$
g(v, K)=g(v)-\frac{(K-1)}{K} X(v) .
$$

It is also useful to note that

$$
\lim _{v \rightarrow 0} \frac{v f(v)}{F(v)}=\lim _{v \rightarrow 0} \frac{f^{\prime}(v) v+f(v)}{f(v)}=1+\phi \quad \text { and } \quad \lim _{v \rightarrow 0} \frac{F(v)}{v f(v)}=\frac{1}{1+\phi}
$$


which will be used repeatedly below.

We now show that

$$
\begin{aligned}
& \lim _{v \rightarrow 0} X(v) v=n \phi+n+2+\frac{\phi+2}{(n-1)(1+\phi)} \\
& \lim _{v \rightarrow 0} Y(v) v=2+\frac{2(\phi+2)}{(n-1)(1+\phi)} .
\end{aligned}
$$

For the first limit, note that

$$
\begin{aligned}
& \lim _{v \rightarrow 0} X(v) v \\
= & \lim _{v \rightarrow 0} \frac{(n-1) F^{n-2}(v) f^{2}(v) v^{2}+F^{n-1}(v) f^{\prime}(v) v^{2}+F^{n-1}(v) f(v) 2 v}{(n-1) f(v) \int_{0}^{v} s F^{n-2}(s) f(s) d s} \\
= & \lim _{v \rightarrow 0} \frac{F^{n-2}(v) f^{2}(v) v^{2}}{f(v) \int_{0}^{v} s F^{n-2}(s) f(s) d s}+\lim _{v \rightarrow 0} \frac{F^{n-1}(v) f^{\prime}(v) v^{2}}{(n-1) f(v) \int_{0}^{v} s F^{n-2}(s) f(s) d s} \\
& +\lim _{v \rightarrow 0} \frac{F^{n-1}(v) f(v) 2 v}{(n-1) f(v) \int_{0}^{v} s F^{n-2}(s) f(s) d s} \\
= & \lim _{v \rightarrow 0} \frac{F^{n-2}(v) f(v) v^{2}}{\int_{0}^{v} s F^{n-2}(s) f(s) d s}+\lim _{v \rightarrow 0} \frac{F^{n-1}(v) f^{\prime}(v) v^{2}}{(n-1) f(v) \int_{0}^{v} s F^{n-2}(s) f(s) d s}+\lim _{v \rightarrow 0} \frac{F^{n-1}(v) 2 v}{(n-1) \int_{0}^{v} s F^{n-2}(s) f(s) d s},
\end{aligned}
$$

where we have used l'Hospital's rule in the first step and then rearranged the expression. The limit of the first term is

$$
\begin{aligned}
\lim _{v \rightarrow 0} \frac{F^{n-2}(v) f(v) v^{2}}{\int_{0}^{v} s F^{n-2}(s) f(s) d s} & =\lim _{v \rightarrow 0} \frac{(n-2) F^{n-3}(v) f^{2}(v) v^{2}+F^{n-2}(v) f^{\prime}(v) v^{2}+F^{n-2}(v) f(v) 2 v}{v F^{n-2}(v) f(v)} \\
& =\lim _{v \rightarrow 0} \frac{(n-2) f^{2}(v) v+F(v) f^{\prime}(v) v+F(v) f(v) 2}{F(v) f(v)} \\
& =\lim _{v \rightarrow 0} \frac{(n-2) f(v) v}{F(v)}+\lim _{v \rightarrow 0} \frac{f^{\prime}(v) v}{f(v)}+2 \\
& =(n-2)(\phi+1)+\phi+2 \\
& =(n-1) \phi+n,
\end{aligned}
$$

where we have have used l'Hospital's rule to obtain the first equality. For the second term we have

$$
\begin{aligned}
\lim _{v \rightarrow 0} \frac{F^{n-1}(v) f^{\prime}(v) v^{2}}{(n-1) f(v) \int_{0}^{v} s F^{n-2}(s) f(s) d s} & =\lim _{v \rightarrow 0} \frac{f^{\prime}(v) v}{f(v)} \frac{F^{n-1}(v) v}{(n-1) \int_{0}^{v} s F^{n-2}(s) f(s) d s} \\
& =\lim _{v \rightarrow 0} \frac{f^{\prime}(v) v}{f(v)} \lim _{v \rightarrow 0} \frac{F^{n-1}(v) v}{(n-1) \int_{0}^{v} s F^{n-2}(s) f(s) d s} \\
& =\phi \lim _{v \rightarrow 0} \frac{(n-1) F^{n-2}(v) f(v) v+F^{n-1}(v)}{(n-1) v F^{n-2}(v) f(v)} \\
& =\phi \lim _{v \rightarrow 0}\left\{1+\frac{F(v)}{(n-1) v f(v)}\right\}
\end{aligned}
$$




$$
=\phi+\frac{1}{n-1} \frac{\phi}{1+\phi} .
$$

The limit for the third term is

$$
\begin{aligned}
\lim _{v \rightarrow 0} \frac{F^{n-1}(v) 2 v}{(n-1) \int_{0}^{v} s F^{n-2}(s) f(s) d s} & =\lim _{v \rightarrow 0} \frac{(n-1) F^{n-2}(v) f(v) 2 v+F^{n-1}(v) 2}{(n-1) v F^{n-2}(v) f(v)} \\
& =\lim _{v \rightarrow 0} \frac{F^{n-2}(v) f(v) 2 v}{v F^{n-2}(v) f(v)}+\lim _{v \rightarrow 0} \frac{F^{n-1}(v) 2}{(n-1) v F^{n-2}(v) f(v)} \\
& =2+\frac{2}{n-1} \lim _{v \rightarrow 0} \frac{F(v)}{v f(v)} \\
& =2+\frac{2}{n-1} \frac{1}{1+\phi} .
\end{aligned}
$$

We can put the three limits together to obtain the desired result.

$$
\begin{aligned}
\lim _{v \rightarrow 0} X(v) v & =((n-1) \phi+n)+\left(\phi+\frac{1}{n-1} \frac{\phi}{1+\phi}\right)+\left(2+\frac{2}{n-1} \frac{1}{1+\phi}\right) \\
& =n \phi+n+2+\frac{\phi+2}{(n-1)(1+\phi)} .
\end{aligned}
$$

For the limit of $Y(v) v$ we have

$$
\begin{aligned}
\lim _{v \rightarrow 0} Y(v) v= & \lim _{v \rightarrow 0} \frac{2 v f(v) \int_{0}^{v} F^{n-1}(s) d s}{(n-1) \int_{0}^{v} s[F(v)-F(s)] F^{n-2}(s) f(s) d s} \\
= & 2 \lim _{v \rightarrow 0} \frac{f(v) \int_{0}^{v} F^{n-1}(s) d s+v f^{\prime}(v) \int_{0}^{v} F^{n-1}(s) d s+v f(v) F^{n-1}(v)}{(n-1) \int_{0}^{v} s F^{n-2}(s) f(s) f(v) d s} \\
= & 2\left\{\lim _{v \rightarrow 0} \frac{\int_{0}^{v} F^{n-1}(s) d s}{(n-1) \int_{0}^{v} s F^{n-2}(s) f(s) d s}+\lim _{v \rightarrow 0} \frac{v f^{\prime}(v)}{f(v)} \frac{\int_{0}^{v} F^{n-1}(s) d s}{(n-1) \int_{0}^{v} s F^{n-2}(s) f(s) d s}\right\} \\
& +2 \lim _{v \rightarrow 0} \frac{v F^{n-1}(v)}{(n-1) \int_{0}^{v} s F^{n-2}(s) f(s) d s} \\
= & 2\left\{\lim _{v \rightarrow 0} \frac{F^{n-1}(v)}{(n-1) v F^{n-2}(v) f(v)}+\phi \lim _{v \rightarrow 0} \frac{F^{n-1}(v)}{(n-1) v F^{n-2}(v) f(v)}\right\} \\
& +2 \lim _{v \rightarrow 0} \frac{F^{n-1}(v)+(n-1) v F^{n-2}(v) f(v)}{(n-1) v F^{n-2}(v) f(v)} \\
= & 2\left\{\lim _{v \rightarrow 0} \frac{F(v)}{(n-1) v f(v)}+\phi \lim _{v \rightarrow 0} \frac{F(v)}{(n-1) v f(v)}+\lim _{v \rightarrow 0} \frac{F(v)}{(n-1) v f(v)}+1\right\} \\
= & 2+2 \frac{2+\phi}{n-1} \lim _{v \rightarrow 0} \frac{F(v)}{v f(v)} \\
= & 2+2 \frac{2+\phi}{n-1} \frac{1}{1+\phi} .
\end{aligned}
$$


Adding up terms we have

$$
\begin{aligned}
& \lim _{v \rightarrow 0} \frac{f^{\prime}(v) v}{f(v)}-\lim _{v \rightarrow 0} X(v) v+\lim _{v \rightarrow 0} Y(v) \\
= & \phi-\left(n \phi+n+2+\frac{\phi+2}{(n-1)(1+\phi)}\right)+\left(2+2 \frac{2+\phi}{n-1} \frac{1}{1+\phi}\right) \\
= & \phi-\frac{((n-1) \phi+n-2)(n \phi+n+1)}{(n-1)(1+\phi)},
\end{aligned}
$$

and hence we have A.6 and A.7.

To show A.8, note that

$$
\begin{aligned}
& \lim _{v \rightarrow 0} r h(v, K) v^{2} \\
= & \frac{K-1}{K} \lim _{v \rightarrow 0} \frac{F^{n-2}(v) f^{2}(v) v^{3}}{\int_{0}^{v} s F^{n-2}(s) f(s)(F(v)-F(s)) d s} \\
= & \frac{K-1}{K} \lim _{v \rightarrow 0} \frac{(n-2) F^{n-3}(v) f^{3}(v) v^{3}+F^{n-2}(v) 2 f(v) f^{\prime}(v) v^{3}+F^{n-2}(v) f^{2}(v) 3 v^{2}}{\int_{0}^{v} s F^{n-2}(s) f(s) f(v) d s} \\
= & \frac{K-1}{K}\left\{\lim _{v \rightarrow 0} \frac{(n-2) F^{n-3}(v) f^{2}(v) v^{3}}{\int_{0}^{v} s F^{n-2}(s) f(s) d s}+\lim _{v \rightarrow 0} \frac{f^{\prime}(v) v}{f(v)} \frac{F^{n-2}(v) 2 f(v) v^{2}}{\int_{0}^{v} s F^{n-2}(s) f(s) d s}+\lim _{v \rightarrow 0} \frac{F^{n-2}(v) f(v) 3 v^{2}}{\int_{0}^{v} s F^{n-2}(s) f(s) d s}\right\} \\
= & \frac{K-1}{K} \lim _{v \rightarrow 0} \frac{(n-2) F^{n-3}(v) f^{2}(v) v^{3}}{\int_{0}^{v} s F^{n-2}(s) f(s) d s}+\frac{K-1}{K}(3+2 \phi) \lim _{v \rightarrow 0} \frac{F^{n-2}(v) f(v) v^{2}}{\int_{0}^{v} s F^{n-2}(s) f(s) d s} .
\end{aligned}
$$

For the first limit we have

$$
\begin{aligned}
\lim _{v \rightarrow 0} \frac{(n-2) F^{n-3}(v) f^{2}(v) v^{3}}{\int_{0}^{v} s F^{n-2}(s) f(s) d s} & =(n-2) \lim _{v \rightarrow 0} \frac{(n-3) F^{n-4}(v) f^{3}(v) v^{3}+F^{n-3}(v) 2 f(v) f^{\prime 3}+F^{n-3}(v) f^{2}(v) 3 v^{2}}{v F^{n-2}(v) f(v)} \\
& =(n-2) \lim _{v \rightarrow 0} \frac{(n-3) f^{2}(v) v^{2}+F(v) 2 f^{\prime 2}+F(v) f(v) 3 v}{F^{2}(v)} \\
& =(n-2) \lim _{v \rightarrow 0} \frac{(n-3) f^{2}(v) v^{2}}{F^{2}(v)}+\frac{2 f(v) v}{F(v)} \frac{f^{\prime}(v) v}{f(v)}+\frac{f(v) 3 v}{F(v)} \\
& =(n-2) \lim _{v \rightarrow 0} \frac{f(v) v}{F(v)}\left((n-3) \frac{f(v) v}{F(v)}+2 \frac{f^{\prime}(v) v}{f(v)}+3\right) \\
& =(n-2)(1+\phi)((n-3)(1+\phi)+2 \phi+3) \\
& =(n+\phi n-2-2 \phi)(n+\phi n-\phi) .
\end{aligned}
$$

For the second limit we have

$$
\begin{aligned}
\lim _{v \rightarrow 0} \frac{F^{n-2}(v) f(v) v^{2}}{\int_{0}^{v} s F^{n-2}(s) f(s) d s} & =\lim _{v \rightarrow 0} \frac{(n-2) F^{n-3}(v) f^{2}(v) v^{2}+F^{n-2}(v) f^{\prime 2}+F^{n-2}(v) f(v) 2 v}{v F^{n-2}(v) f(v)} \\
& =\lim _{v \rightarrow 0} \frac{(n-2) F^{n-3}(v) f^{2}(v) v^{2}}{v F^{n-2}(v) f(v)}+\lim _{v \rightarrow 0} \frac{F^{n-2}(v) f^{\prime 2}}{v F^{n-2}(v) f(v)}+\lim _{v \rightarrow 0} \frac{F^{n-2}(v) f(v) 2 v}{v F^{n-2}(v) f(v)}
\end{aligned}
$$




$$
\begin{aligned}
& =(n-2) \lim _{v \rightarrow 0} \frac{f(v) v}{F(v)}+\lim _{v \rightarrow 0} \frac{f^{\prime}(v) v}{f(v)}+2 \\
& =(n-2)(1+\phi)+\phi+2 \\
& =n+n \phi-\phi .
\end{aligned}
$$

Hence we have

$$
\begin{aligned}
\lim _{v \rightarrow 0} r h(v, K) v^{2} & =\frac{K-1}{K}(n+\phi n-2-2 \phi)(n+\phi n-\phi)+\frac{K-1}{K}(3+2 \phi)(n+n \phi-\phi) \\
& =\frac{K-1}{K}(n+\phi n+1)(n+\phi n-\phi) .
\end{aligned}
$$

With this notation, we can give a sufficient condition for the existence of a feasible solution to the ODE in (4.1), and we can also provide a sufficient condition under which such a feasible solution does not exist. It turns out that these two sufficient conditions are almost mutually exclusive, depending on whether $\kappa=\lim _{v \rightarrow 0} g(v) v$ is above or below -1 .

Lemma 15. (i) If $\kappa<-1$, there exists no decreasing solution to 4.1.

(ii) If $\kappa>-1$, there exists a decreasing solution to (4.1).

(iii) Among all such solutions (4.2) maximizes the seller's revenue for a given boundary value $v_{0}^{+}$.

Proof. As in the proof of Lemma 13, we apply the change of variables $\dot{v}_{t}=y$ and consider the equation

$$
y^{\prime}(v)+g(v) y(v)+r=0 .
$$

The general solution is given by

$$
y(v)=e^{-\int_{m}^{v} g(x) d x}\left(C-\int_{m}^{v} r e^{\int_{m}^{w} g(x) d x} d w\right),
$$

where $m>0{ }^{26}$ Feasibility requires that $y(v)<0$ for all $v \in\left(0, v_{0}^{+}\right)$.

(i) Suppose $\kappa<-1$. Since $\kappa=\lim _{v \rightarrow 0} g(v) v$, there must exist $\gamma>0$ such that $g(v) \leq-\frac{1}{v}$ for all $v \in(0, \gamma]$. Then there does not exist a finite $C$ such that the general solution in A.9 satisfies $y(v)<0$ for all $v \in\left(0, v_{0}^{+}\right)$. Suppose the opposite is true. Then there must exist a finite $C$ such that, for all $v \in\left(0, v_{0}^{+}\right)$,

$$
C<\int_{m}^{v} r e^{\int_{m}^{w} g(x) d x} d w
$$

\footnotetext{
${ }^{26}$ For $m=0$, the solution candidate is not well defined for all $\kappa$ because the first integral evaluates to $\infty$.
} 
Since the right-hand side is increasing in $v$ this implies

$$
\lim _{v \rightarrow 0} \int_{m}^{v} r e^{\int_{m}^{w} g(x) d x} d w>-\infty
$$

We may assume that $0<m<\gamma$. In this case, the limit can be computed as follows:

$$
\begin{aligned}
\lim _{v \rightarrow 0} \int_{m}^{v} r e^{\int_{m}^{w} g(x) d x} d w & =\lim _{v \rightarrow 0}-\int_{v}^{m} r e^{-\int_{w}^{m} g(x) d x} d w \\
& \leq \lim _{v \rightarrow 0}-\int_{v}^{m} r e^{\int_{w}^{m} \frac{1}{x} d x} d w \\
& =\lim _{v \rightarrow 0}-\int_{v}^{m} r \frac{m}{w} d w \\
& =-\infty
\end{aligned}
$$

A contradiction. This shows part (i).

To prove part (ii), we first set

$$
C=-\int_{0}^{m} r e^{\int_{m}^{w} g(x) d x} d w
$$

and show that the resulting solution

$$
y(v)=-e^{-\int_{m}^{v} g(x) d x} \int_{0}^{v} r e^{\int_{m}^{w} g(x) d x} d w=-\int_{0}^{v} r e^{-\int_{w}^{v} g(x) d x} d w
$$

is negative and finite for all $v$. It is clear that $y(v)<0$, so it suffices to rule out $y(v)=-\infty$. Since $\kappa=\lim _{v \rightarrow 0} g(v) v>-1$, there exist $\hat{\kappa}>-1$ and $\gamma>0$ such that $g(v) \geq \frac{\hat{\kappa}}{v}$ for all $v \in(0, \gamma]$. Hence the limit in A.10 can be computed as (where we may again assume that $0<m<\gamma$ ):

$$
\begin{aligned}
\lim _{v_{t} \rightarrow 0} \int_{m}^{v_{t}} r e^{\int_{m}^{v} g(x) d x} d v & =\lim _{v_{t} \rightarrow 0}-\int_{v_{t}}^{m} r e^{-\int_{v}^{m} g(x) d x} d v \\
& \geq \lim _{v_{t} \rightarrow 0}-\int_{v_{t}}^{m} r e^{-\hat{\kappa} \ln \frac{m}{v}} d v \\
& =\lim _{v_{t} \rightarrow 0}-\int_{v_{t}}^{m} r\left(\frac{v}{m}\right)^{\hat{\kappa}} d v \\
& =-r m^{-\hat{\kappa}} \frac{1}{\hat{\kappa}+1} \lim _{v_{t} \rightarrow 0}\left(m^{\hat{\kappa}+1}-v_{t}^{\hat{\kappa}+1}\right) \\
& >-\infty .
\end{aligned}
$$

Therefore, $y(v)$ is finite and $y(v)<0$ for all $v$. Next we have to show that A.12 can be integrated to obtain a feasible solution of the auxiliary problem. It suffices to verify that the following boundary condition

$$
\lim _{t \rightarrow \infty} v_{t}=0
$$


is satisfied. Recall that $\dot{v}_{t}=y\left(v_{t}\right)$. Therefore, we have

$$
\dot{v}_{t}=-e^{-\int_{m}^{v_{t}} g(v) d v}\left(\int_{0}^{v_{t}} r e^{\int_{m}^{v} g(x) d x} d v\right)
$$

We first show that, for any $v_{0}^{+} \in[0,1]$, the solution to this differential equation satisfies (A.13). Since the term in the parentheses is strictly positive we have

$$
\frac{e^{\int_{m}^{v} g(v) d v} \dot{v}_{t}}{\int_{0}^{v_{t}} e^{\int_{m}^{v} g(x) d x} d v}=-r
$$

Integrating both sides we get

$$
\ln \int_{0}^{v_{t}} e^{\int_{m}^{v} g(x) d x} d v-\ln \int_{0}^{v_{0}} e^{\int_{m}^{v} g(x) d x} d v=-r t
$$

Now take $t \rightarrow \infty$. The RHS diverges to $-\infty$ and the second term on the LHS is constant, so we must have

$$
\lim _{t \rightarrow \infty} \ln \int_{0}^{v_{t}} e^{\int_{m}^{v} g(x) d x} d v=-\infty
$$

which is possible only if $\lim _{t \rightarrow \infty} v_{t}=0$. Therefore, we have found a solution that satisfies the boundary condition and is decreasing for all starting values $v_{0}^{+}$. This concludes the proof for part (ii).

To prove part (iii), it suffices to rule out the possibility that other solutions may yield a higher value of the objective function. In light of A.10, any decreasing solution must satisfy A.9 with

$$
C=-\hat{C}-\int_{0}^{m} r e^{\int_{m}^{w} g(x) d x} d w
$$

where $\hat{C} \geq 0$, because $\hat{C}<0$ implies $y(v)>0$ for $v$ sufficiently small. Notice that if $\hat{C}=0, \dot{v}_{t}$ is given by A.12:

$$
\dot{v}_{t}=-e^{-\int_{m}^{v_{t}} g(v) d v} \int_{0}^{v_{t}} r e^{\int_{m}^{v} g(x) d x} d v=-\int_{0}^{v_{t}} r e^{-\int_{v}^{v_{t}} g(x) d x} d v
$$

Let $y$ denote the solution for $\hat{C}=0$ and $z$ denote the solution for some $\hat{C}>0$. If $\hat{C}>0$, then we have for all $v \in(0,1]$ :

$$
z(v)=y(v)-\hat{C} e^{-\int_{m}^{v} g(x) d x}<y(v) .
$$

Let $v_{t}$ be the cutoff path for $\hat{C}=0$ and $w_{t}$ be the cutoff path for $\hat{C}>0$. If we fix $v_{0}=w_{0}$, then $z(v)<y(v)$ implies that for all $t>0, w_{t}<v_{t}$. To see this, note that whenever $v_{t}=w_{t} \neq 0$, we have $\dot{w}_{t}=z\left(w_{t}\right)<y\left(v_{t}\right)=\dot{v}_{t}$. Hence, at every point where the two cutoff paths coincide, $w_{t}$ must cross $v_{t}$ from above. But since $w_{0}=v_{0}$, this cannot happen (except at $t=0$ ). As a result, $w_{t}$ cannot be 
part of the optimal solution 27 Therefore, the optimal solution is given by $\hat{C}=0$.

Now we are ready to prove part (i) of Theorem 2 .

Proof of Part (i) of Theorem 2. Part (ii) of Lemma 15 shows that, if $\kappa>-1$, then there exists a feasible solution to the auxiliary problem that differs from the efficient auction. The result, together with Proposition 3, implies that the efficient auction is not optimal if $\kappa>-1$. If $\phi>-1$, the condition $\kappa>-1$ is equivalent to

$$
(1+\phi)^{2}(n-1)-((n-1) \phi+n-2)(n \phi+n+1)>0 .
$$

By collecting terms with respect to $n$, we can change the condition into

$$
-(\phi+1)^{2} n^{2}+2(\phi+1)^{2} n-\left(\phi^{2}+\phi-1\right)>0,
$$

or equivalently

$$
n<1+\frac{\sqrt{2+\phi}}{1+\phi}=\bar{N}(F)
$$

This proves part (i) of the Theorem.

\section{A.3.3 Step 3: Prove Optimality of the Constructed Solution}

To prove part (ii) of Theorem 2, we first observe that $v(1-F(v))$ is always locally concave when $v$ is sufficiently close to zero. To see this, note that the concavity of $v(1-F(v))$ is equivalent to

$$
\frac{f^{\prime}(v) v}{f(v)} \geq-2
$$

It follows from Remark 1 that $v(1-F(v))$ is locally concave for $v$ sufficiently close to zero. Now it suffices to show the following key result which is proved in Lemmas 19 and 20: the payoff floor must be binding at the optimal solution whenever $v(1-F(v))$ is concave. This is sufficient for part (ii) of Theorem 2, because the requirement that the optimal solution must have a binding payoff floor constraint if $v_{t}$ is small, together with the impossibility of such a solution if $n>\bar{N}(F)$ implied by part (i) of Lemma 15 , leaves only the efficient auction as an optimal solution.

The result that a concave monopoly profit implies a binding payoff floor constraint is also crucial for proving Theorem 3. It implies that for the optimal solution, the payoff floor constraint can only

\footnotetext{
${ }^{27}$ If $J\left(v_{0}\right)<0$, then the cutoff path $v_{t}$ leads to later trading times for types with negative virtual valuation, hence the sellers expected profit is higher. Next suppose that $J\left(v_{0}\right)>0$. Let $x$ be defined by $J(x)=0$. Let $s_{v}$ be the time where $v_{s}=x$ and $s_{w}$ be the time where $w_{s}=x$. Since $w_{t}<v_{t}$ for all $t$, we must have $s_{w}<s_{v}$. Now we construct a new feasible cutoff path that yields a higher expected profit than $w$. For the new path we set $\hat{v}_{0}=v_{\left(s_{v}-s_{w}\right)}$ and $\dot{\hat{v}}_{t}=\dot{v}_{\left(s_{v}-s_{w}\right)}$. Notice that $\hat{v}_{t}<w_{t}$ for $t<s_{w}, \hat{v}_{t}>w_{t}$ for $t>s_{w}$, and $\hat{v}_{s_{w}}=w_{s_{w}}=x$. Hence, with the new cutoff paths, all types with $J(v)>0$ trade (weakly) earlier and all types with $J(v)<0$ trade (strictly) later that with the old cutoff path. Therefore the expected revenue of the seller is strictly higher.
} 
be slack on intervals where $v(1-F(v))$ is non-concave. If we impose Assumption A4 which requires global concavity, then the solution constructed in Lemma 15 (with an appropriately chosen starting cutoff $v_{0}^{+}$) is optimal if $n<\bar{N}(F)$, which shows Theorem 3 .

To prove this key result, it suffices to show that feasible solutions for which the payoff floor constraint is slack for a time interval $(a, b)$ are never optimal if $v(1-F(v))$ is concave on the interval of valuations $\left[v_{b}, v_{a}\right]$ that trade between $a$ and $b$. Specifically, suppose we have a feasible solution $T$ with corresponding cutoff path $v_{t}$ for which the payoff floor constraint is strictly slack for all $t \in(a, b)$ where $0 \leq a<b$. We want to construct a new feasible solution $\hat{T}$ with corresponding cutoff path $\hat{v}_{t}$ that is also feasible and improves the seller's expected profit. Our construction will only change the trading times of the valuations in the interval $\left(v_{b}^{+}-\varepsilon, v_{a}\right)$ where $\varepsilon>0$ can be arbitrarily small. This implies that the new solution satisfies the payoff floor constraint for all $t$ for which $\hat{v}_{t}<v_{b}^{+}-\varepsilon$ because the continuation is unchanged for such $t$. For times $t$ such that $\hat{v}_{t} \in\left(v_{b}^{+}-\varepsilon, v_{a}\right)$, we exploit that the payoff floor constraint was slack before the modification. This implies that a small variation in trading times will not lead to a violation of the payoff floor constraint by the new solution. Depending on whether types trade in the interior of the slack interval or types trade only at the end of the interval, the constructed variations are different and are covered in Lemmas 19 and 20, respectively. Finally, we exploit the following lemma to show that the payoff floor constraint for $t<a$ remains satisfied.

Lemma 16. Let $T$ and $\hat{T}$ be non-increasing solutions with corresponding cutoff paths $v_{t}$ and $\hat{v}_{t}$ such that $v_{t}=\hat{v}_{t}$ for $t \leq a$. Suppose $T$ is feasible and that the slack in the payoff floor constraint at $a$ is the same for $T$ and $\hat{T}$. If the ex-ante revenue of the seller under $\hat{T}$ is greater than or equal to the revenue under $T$, then $\hat{T}$ satisfies the payoff floor constraint for all $t \leq a$.

Proof. If the seller's revenue is higher under $\hat{T}$, then

$$
\int_{0}^{1}\left(e^{-r \hat{T}(v)}-e^{-r T(v)}\right)\left(v-\frac{1-F(v)}{f(v)}\right) n F(v)^{n-1} f(v) d v>0 .
$$

Using the assumption that $v_{t}=\hat{v}_{t}$ for all $t \leq a$ and hence $T(v)=\hat{T}(v)$ for all $v>v_{a}$, we can rewrite this expression as

$$
\int_{0}^{v_{a}}\left(e^{-r \hat{T}(v)}-e^{-r T(v)}\right)\left(v-\frac{1-F(v)}{f(v)}\right) n F(v)^{n-1} f(v) d v>0 .
$$

Since both cutoff sequences have the same slack in the payoff floor constraint at $a$ and $v_{a}=\hat{v}_{a}$, we have

$$
\int_{0}^{v_{a}}\left(e^{-r \hat{T}(v)}-e^{-r T(v)}\right)\left(v-\frac{F\left(v_{a}\right)-F(v)}{f(v)}\right) n F(v)^{n-1} f(v) d v=0 .
$$


Subtracting equation A.15 from inequality A.14 we obtain

$$
\int_{0}^{v_{a}}\left(e^{-r \hat{T}(v)}-e^{-r T(v)}\right)\left(\frac{F\left(v_{a}\right)-1}{f(v)}\right) n F(v)^{n-1} f(v) d v>0,
$$

which is equivalent to, for all $t<a$,

$$
\int_{0}^{v_{a}}\left(e^{-r \hat{T}(v)}-e^{-r T(v)}\right)\left(\frac{F\left(v_{a}\right)-F\left(v_{t}\right)}{f(v)}\right) n F(v)^{n-1} f(v) d v>0
$$

Adding equality A.15 to the above inequality, we get

$$
\int_{0}^{v_{a}}\left(e^{-r \hat{T}(v)}-e^{-r T(v)}\right)\left(v-\frac{F\left(v_{t}\right)-F(v)}{f(v)}\right) n F(v)^{n-1} f(v) d v>0 .
$$

But this means that the slack in the payoff floor constraint at $t<a$ is strictly greater under $\hat{T}$ than for $T$. Hence, the payoff floor constraint is fulfilled under $\hat{T}$ for all $t<a$.

In light of Lemma 16, we construct the new solution in such a way that the payoff floor constraint at is $a$ unchanged and ex-ante revenue is improved. The Lemma then shows that the payoff floor constraint is fulfilled for all $t \in[0, a]$ for the new solution.

Before we take this approach, we prove two observations that will be useful in the subsequent proofs. First, concavity of the monopoly profit is equivalent to the monotonicity of $J(v) f(v)$ or the monotonicity of $J(v \mid v \leq x) f(v)$ for all $x \in[0,1]$, as shown in the following lemma.

Lemma 17. Suppose $v(1-F(v))$ is strictly concave for on an interval $[a, b]$ where $a<b \leq x$. Then $(J(s \mid v \leq x)) f(s)$ is strictly increasing in $s$ on the interval $[a, b]$.

Proof. Note that

$$
\begin{aligned}
J(s \mid v \leq x) f(s) & =\left(s-\frac{F(x)-F(s)}{f(s)}\right) f(s) \\
& =\left(s-\frac{1-F(s)}{f(s)}\right) f(s)+1-F(x) \\
& =J(s) f(s)+(1-F(x)) .
\end{aligned}
$$

Hence, $J(s \mid v \leq x) f(s)$ is strictly increasing in $s$ if and only if $J(s) f(s)$ is strictly increasing.

$$
\frac{d}{d s}(J(s) f(s))=\frac{d}{d s}(s f(s)-(1-F(s)))=-\frac{d^{2}}{d s^{2}}(s(1-F(s))) .
$$

Second, we show that, whenever the payoff floor constraint is slack for an interval $(a, b)$, the types that trade within the interval must have positive virtual valuation evaluated at any point of 
the time interval. Otherwise, one can construct alternative feasible trading times that delay the trade for types with negative virtual valuation and increase revenue.

Lemma 18. Let $T$ be an optimal solution for which the payoff floor constraint is slack for all $t \in(a, b)$. Then $J_{t}(v) \geq 0$ for all $t \in(a, b]$ and $v \in\left[v_{b^{+}}, v_{a}\right]$. If $v_{t}$ is continuous at $a, J_{a}(v) \geq 0$ for all $v \in\left[v_{b^{+}}, v_{a}\right]$.

Proof. Let us first assume that the payoff floor constraint is strictly slack for all $t \in[a, b]$. Suppose by contradiction that $J_{t}(v)<0$ for some $t \in(a, b]$ and some $v \in\left[v_{b^{+}}, v_{a}\right]$. Then $J_{t}(\tilde{v})<0$ for some $\tilde{v} \in\left[v_{b^{+}}, v_{a}\right]$ and all $t \leq a$, since $J_{t}(v)$ is non-decreasing in $t$. We claim that the following modification is feasible and improves revenue for $\delta>0$ sufficiently small:

$$
\hat{T}(v)=\left\{\begin{array}{ll}
T(v), & \text { if } v>\tilde{v} \\
T(v)+\delta, & \text { if } v \leq \tilde{v}
\end{array} .\right.
$$

With the new trading times, the payoff floor constraint at $t+\delta$ for $t \geq T(\tilde{v})$ is the same as the payoff floor constraint for $t$ at the original trading times. For $t<a$ the RHS of the payoff floor constraint is unchanged and the LHS is increased because we delay trade of types that have a negative virtual valuation at $t \leq a$. For $a<t<T(\tilde{v})+\delta$, we distinguish two cases. If the type $\tilde{v}$ is the only type that trades at $T(\tilde{v})$ then for $\delta$ sufficiently small, the payoff floor constraint is fulfilled because it was strictly slack for $a \leq t<T(\tilde{v})$ before the change. If $\tilde{v}$ is part of an atom of types that all trade at the same time, the same argument applies to the payoff floor constraint at $t \in[a, T(\tilde{v})]$. After the modification, however, the posterior at times $t \in(T(\tilde{v}), T(\tilde{v})+\delta)$ is the prior truncated to [0, $]$. Before the change, this posterior did not arise on the equilibrium path. By Lemma 11 we have.

$$
\int_{0}^{\tilde{v}}\left(e^{-r(T(v)-T(\tilde{v}))}-1\right)\left(v-\frac{F(\tilde{v})-F(v)}{f(v)}\right) f(v) n(F(v))^{n-1} d v>0 .
$$

This implies that after the modification, the payoff floor constraint is strictly slack at $T(\tilde{v})+\delta$ and hence for $\delta$ sufficiently small it is also fulfilled for all $t \in(T(\tilde{v}), T(\tilde{v})+\delta)$.

It remains to show the result for the case that the payoff floor constraint is binding at $a$ and $b$ but strictly slack on $(a, b)$. In this case, we know that the result holds true for $t \in[a+\varepsilon, b-\varepsilon]$ for any $\varepsilon>0$ and $v \in\left[v_{b-\varepsilon}^{+}, v_{a+\varepsilon}\right]$. Since $v-\frac{F\left(v_{t}\right)-F(v)}{f(v)}$ is continuous in $v_{t}$ and $v$ and there is no atom at $a$ or $b$, respectively if the payoff floor constraint binds, $J_{t}(v)$ is continuous in $(t, v)$ at the endpoints of the interval if the payoff floor constraint is binding. By continuity, the result for $[a+\varepsilon, b-\varepsilon]$ extends to the endpoints.

Now we construct a feasible variation that improves revenue. We have to consider two scenarios. In the first scenario, there is a time interval $\left[s, s^{\prime}\right] \subset(a, b)$ such that trade occurs with positive probability between $s$ and $s^{\prime}$. In this case, there exists a variation of the trading times for those 
types who trade in the interval $\left[s, s^{\prime}\right]$. Roughly speaking, we construct an alternative solution by splitting the types trading in $\left(s, s^{\prime}\right)$, and then clustering them to the endpoints $s$ and $s^{\prime}$. In particular, we advance the trading time of high types who previously traded in $\left(s, s^{\prime}\right)$ and delay the trading times of low types who previously traded in $\left(s, s^{\prime}\right)$. The variation is constructed such that the payoff floor constraint at $s$ is unchanged. Furthermore, our concavity assumption ensures that the alternative trading time $\hat{T}$ also leads to a higher ex ante revenue than $T$. It follows from Lemma 16, that the payoff floor constraint is fulfilled for all $t<s$. Formally, we have the following result.

Lemma 19. Let $T$ be a feasible solution for which the payoff floor constraint is strictly slack for all $t \in(a, b)$. Suppose there is a positive measure of types $v \in\left[v_{b}, v_{a}\right]$ for which $T(v) \notin\{a, b\}$. If $v(1-F(v))$ is strictly concave for all $v \in\left[v_{b}, v_{a}\right]$, then $T$ is not optimal.

Proof. Suppose by contradiction that $T$ is optimal. We consider a variation of the trading times on small interval $\left[s, s^{\prime \prime}\right] \subset(a, b)$ constructed as follows. First we choose $\left[s, s^{\prime \prime}\right]$ such that $v_{s^{\prime \prime}}<v_{s}$ and there is a positive measure of types with trading times in $\left(s, s^{\prime \prime}\right)$ and such that there are no atoms of trade at $s$ or $s^{\prime \prime}$. In words, we choose an interval of types that do not trade at the endpoints of $\left[s, s^{\prime \prime}\right]$ (they could all trade in an atom). Next we pick some type $w$ with $s<T(w)<s^{\prime \prime}$ and define $\hat{T}$ such that all types in $\left[v_{s^{\prime \prime}}, w\right]$ trade at $s^{\prime \prime}$ and all types in $\left(w, v_{s}\right]$ trade at $s$. Formally the modification of the trading time can be written as

$$
\hat{T}(v)= \begin{cases}T(v) & \text { if } v \geq v_{s} \\ s & \text { if } v \in\left(w, v_{s}\right] \\ s^{\prime \prime} & \text { if } v \in\left[v_{s^{\prime \prime}}, w\right] \\ T(v) & \text { if } v \leq v_{s^{\prime \prime}} .\end{cases}
$$

We want so choose $w$ such that the payoff floor constraint at time $s$ remains unchanged, formally:

$$
\int_{w}^{v_{s}}\left(e^{-r s}-e^{-r T(v)}\right)\left(J_{s}(v) f(v)\right) n(F(v))^{n-1} d v+\int_{v_{s^{\prime \prime}}}^{w}\left(e^{-r s^{\prime \prime}}-e^{-r T(v)}\right)\left(J_{s}(v) f(v)\right) n(F(v))^{n-1} d v=0 .
$$

Note that if $w=v_{s}$ the first integral vanishes so that the LHS is negative, and if $w=v_{s^{\prime \prime}}$, the second integral vanishes and the LHS is positive. Since the LHS is continuous in $w$, we can choose $w$ such that A.16 is satisfied. Note also that if we choose $s$ and $s^{\prime \prime}$ sufficiently close together, then the payoff-floor constraint remains satisfied for all $t \in\left[s, s^{\prime \prime}\right]$ because it was strictly slack before the variation. Also the payoff-floor constraint for $t>s^{\prime \prime}$ is not affected by this change. Finally, if we can show that the ex-ante revenue increases, Lemma 16 implies that the payoff floor constraint is 
also satisfied for $t<s$. The ex-ante revenue is increased if

$\int_{w}^{v_{s}}\left(e^{-r s}-e^{-r T(v)}\right)(J(v) f(v)) n(F(v))^{n-1} d v+\int_{v_{s^{\prime \prime}}}^{w}\left(e^{-r s^{\prime \prime}}-e^{-r T(v)}\right)(J(v) f(v)) n(F(v))^{n-1} d v>0$.

By subtracting A.16 from the above inequality, we get

$\int_{w}^{v_{s}}\left(e^{-r s}-e^{-r T(v)}\right)\left(F\left(v_{s}\right)-1\right) n(F(v))^{n-1} d v+\int_{v_{s^{\prime \prime}}}^{w}\left(e^{-r s^{\prime \prime}}-e^{-r T(v)}\right)\left(F\left(v_{s}\right)-1\right) n(F(v))^{n-1} d v>0$,

which is equivalent to

$$
\int_{w}^{v_{s}}\left(e^{-r s}-e^{-r T(v)}\right) n(F(v))^{n-1} d v+\int_{v_{s^{\prime \prime}}}^{w}\left(e^{-r s^{\prime \prime}}-e^{-r T(v)}\right) n(F(v))^{n-1} d v<0 .
$$

Multiplying by $J_{s}(w) f(w)$ we have (by Lemma $18, J_{s}\left(s^{\prime \prime}\right) \geq 0$ and hence $J_{s}(w)>0$.)

$\int_{w}^{v_{s}}\left(e^{-r s}-e^{-r T(v)}\right) J_{s}(w) f(w) n(F(v))^{n-1} d v+\int_{v_{s^{\prime \prime}}}^{w}\left(e^{-r s^{\prime \prime}}-e^{-r T(v)}\right) J_{s}(w) f(w) n(F(v))^{n-1} d v<0$.

By Lemma 17, we have that $J_{s}(v) f(v)$ is strictly increasing. This implies

$$
\begin{aligned}
& \int_{w}^{v_{s}} \underbrace{\left(e^{-r s}-e^{-r T(v)}\right)}_{>0} J_{s}(w) f(w) n(F(v))^{n-1} d v+\int_{v_{s^{\prime \prime}}}^{w} \underbrace{\left(e^{-r s^{\prime \prime}}-e^{-r T(v)}\right)}_{<0} J_{s}(w) f(w) n(F(v))^{n-1} d v \\
& <\int_{w}^{v_{s}}\left(e^{-r s}-e^{-r T(v)}\right) J_{s}(v) f(v) n(F(v))^{n-1} d v+\int_{v_{s^{\prime \prime}}}^{w}\left(e^{-r s^{\prime \prime}}-e^{-r T(v)}\right) J_{s}(v) f(v) n(F(v))^{n-1} d v \\
& =0
\end{aligned}
$$

where the last equality follows from A.16.

Lemma 19 implies that the probability of trade at times in the interior of the slack interval must be zero. It leaves open the scenario in which the slack interval consists of a single "quiet period" without trade in $(a, b)$ followed by a single "atom" at $b$, formally, $v_{a}=v_{b}>v_{b}^{+}$. In this case, we construct an alternative trading scheme by splitting the atom so that the trading times of high types in the atom are advanced, while the trading times of low types in the atom are delayed. The latter requires that we also delay the trading time for types $v \in\left[v_{b}^{+}-\varepsilon, v_{b}^{+}\right]$for some $\varepsilon>0$. Otherwise the new solution would violate monotonicity of the trading times. This modification can be constructed in a way such that the slack in the payoff floor constraint at $a$ remains unchanged and the payoff floor constraint is satisfied on the newly created second quiet period. Again, concavity implies that ex-ante revenue is increased by this variation which implies that the payoff floor constraint at $t \leq a$ is still satisfied after the variation. Formally, we have the following result.

Lemma 20. Let $T$ be a feasible solution for which the payoff floor constraint is strictly slack for 
all $t \in(a, b]$ and binding for $a$ and $b_{+}$. Suppose $T(v)=b$ for all $v \in\left(v_{b}^{+}, v_{a}\right)$. If $v(1-F(v))$ is strictly concave for all $v \in\left[v_{b_{+}}-\varepsilon, v_{b}\right]$ for some $\varepsilon>0$, then $T$ is not optimal.

Proof. The logic of the proof is similar to proof of Lemma 19. Again, suppose by contradiction that $T$ is optimal. We construct a variation by splitting the atom at some type $w \in\left(v_{b^{+}}, v_{b}\right)$. First, we let types $\left[w, v_{b}\right]$ trade at $s<b$. Second, we want to delay the trading times for types $\left[v_{b^{+}}, w\right)$ to $s^{\prime \prime}>b$, where $v_{s^{\prime \prime}} \geq v_{b^{+}}-\varepsilon$. In order to maintain monotonicity we also have to delay the trading time of all types $v \in\left[v_{s^{\prime \prime}}, v_{b^{+}}\right)$. To summarize we have:

$$
\hat{T}(v)= \begin{cases}T(v) & \text { if } v>v_{b}, \\ s & \text { if } v \in\left[w, v_{b}\right], \\ s^{\prime \prime} & \text { if } v \in\left(v_{s^{\prime \prime}}, w\right), \\ T(v) & \text { if } v \leq v_{s^{\prime \prime}}\end{cases}
$$

We choose $w, s$, and $s^{\prime \prime}$ such that the payoff floor constraint at $a$ is unchanged:

$$
\begin{gathered}
\int_{w}^{v_{b}}\left(e^{-r s}-e^{-r b}\right)\left(J_{a}(v) f(v)\right) n(F(v))^{n-1} d v \\
+\int_{v_{b^{+}}}^{w}\left(e^{-r s^{\prime \prime}}-e^{-r b}\right)\left(J_{a}(v) f(v)\right) n(F(v))^{n-1} d v \\
+\int_{v_{s^{\prime \prime}}}^{v_{b^{+}}}\left(e^{-r s^{\prime \prime}}-e^{-r T(v)}\right)\left(J_{a}(v) f(v)\right) n(F(v))^{n-1} d v=0 .
\end{gathered}
$$

We argue that it is feasible to choose such $w, s$, and $s^{\prime \prime}$. First note that, if we set $s^{\prime \prime}=b, v_{s^{\prime \prime}}=v_{b}^{+}$, and $s<b$, the left hand side of the equality is strictly positive since $J_{a}(w) \geq 0$ by Lemma 18 . Next, we show that for $s=b$ we can choose $s^{\prime \prime}>b$ such that the left hand side of the expression is strictly negative. If $J_{a}\left(v_{b^{+}}\right) f\left(v_{b^{+}}\right)>0$, we can choose $s^{\prime \prime}$ such that $J_{a}\left(v_{s^{\prime \prime}}\right) f\left(v_{s^{\prime \prime}}\right) \geq 0$. In this case the last two integrals are strictly negative. If $J_{a}\left(v_{b^{+}}\right) f\left(v_{b^{+}}\right)=0("<"$ is ruled out by Lemma 18$)$ then $J_{a}(v) f(v)<0$ for $v<v_{b}^{+}$, and we have

$$
\begin{aligned}
& \int_{v_{b^{+}}}^{w}\left(e^{-r s^{\prime \prime}}-e^{-r b}\right)\left(J_{a}(v) f(v)\right) n(F(v))^{n-1} d v \\
& +\int_{v_{s^{\prime \prime}}}^{v_{b^{+}}}\left(e^{-r s^{\prime \prime}}-e^{-r T(v)}\right)\left(J_{a}(v) f(v)\right) n(F(v))^{n-1} d v \\
\leq & \int_{v_{b^{+}}}^{w}\left(e^{-r s^{\prime \prime}}-e^{-r b}\right)\left(J_{a}(v) f(v)\right) n(F(v))^{n-1} d v \\
& +\int_{v_{s^{\prime \prime}}}^{v_{b^{+}}}\left(e^{-r s^{\prime \prime}}-e^{-r b}\right)\left(J_{a}(v) f(v)\right) n(F(v))^{n-1} d v \\
= & \left(e^{-r s^{\prime \prime}}-e^{-r b}\right)\left[\int_{v_{b^{+}}}^{w}\left(J_{a}(v) f(v)\right) n(F(v))^{n-1} d v+\int_{v_{s^{\prime \prime}}}^{v_{b^{+}}}\left(J_{a}(v) f(v)\right) n(F(v))^{n-1} d v\right]
\end{aligned}
$$




$$
\leq\left(e^{-r s^{\prime \prime}}-e^{-r b}\right)\left[\int_{v_{b^{+}}}^{w}\left(J_{a}(v) f(v)\right) n(F(v))^{n-1} d v+\left(J_{a}\left(v_{s^{\prime \prime}}\right) f\left(v_{s^{\prime \prime}}\right)\right) \int_{v_{s^{\prime \prime}}}^{v_{b^{+}}} n(F(v))^{n-1} d v\right] .
$$

We want to show that for some $s^{\prime \prime}$

$$
\int_{v_{b^{+}}}^{w}\left(J_{a}(v) f(v)\right) n(F(v))^{n-1} d v+\left(J_{a}\left(v_{s^{\prime \prime}}\right) f\left(v_{s^{\prime \prime}}\right)\right) \int_{v_{s^{\prime \prime}}}^{v_{b}+} n(F(v))^{n-1} d v>0 .
$$

Note that $\left(J_{a}\left(v_{s^{\prime \prime}}\right) f\left(v_{s^{\prime \prime}}\right)\right) \int_{v_{s^{\prime \prime}}}^{v_{b}} n(F(v))^{n-1} d v$ is continuous in $v_{s^{\prime \prime}}$. Hence, there is a $v_{s^{\prime \prime}}<v_{b^{+}}$such that

$$
\int_{v_{b^{+}}}^{w}\left(J_{a}(v) f(v)\right) n(F(v))^{n-1} d v+\left(J_{a}\left(v_{s^{\prime \prime}}\right) f\left(v_{s^{\prime \prime}}\right)\right) \int_{v_{s^{\prime \prime}}}^{v_{b^{+}}} n(F(v))^{n-1} d v>0 .
$$

Moreover, for every $v_{s^{\prime \prime}}<v_{b}$ there is an $\hat{s}$ with $v_{\hat{s}} \in\left(v_{s^{\prime \prime}}, v_{b}\right)$ such that there is no atom at $\hat{s}$. Hence we can take $v_{s^{\prime \prime}}$ be a type that is not part of an atom. To summarize, we have shown that for some $(s, b)$ the payoff floor constraints at $a$ decreases and for some $\left(b, s^{\prime \prime}\right)$ it increases. We can select $s^{\prime \prime}$ such that the last two integrals in A.17 become arbitrary small. Since the first integral is continuous in $s$ we can find a value for $s$ such that the whole expression is equal to zero. This proves that our construction is possible.

If the payoff-floor constraint binds at $a$ it must be slack for all $t \in(a, s]$ since there is no trade in this interval. Next we argue that the variation does not violate the payoff floor constraint for $t>s$. If we choose both $s$ and $s^{\prime \prime}$ sufficiently close to $b$, then the payoff-floor constraint remains satisfied for all $t \in\left(s, s^{\prime \prime}\right]$ because for every $v_{b^{+}}<w<v_{b}$ Lemma 11 implies

$$
\int_{0}^{w}\left(e^{-r(T(v)-b)}-1\right)\left(v-\frac{F(w)-F(v)}{f(v)}\right) f(v) n(F(v))^{n-1} d v>0 .
$$

Also the payoff-floor constraint for $t>s^{\prime \prime}$ is not affected by this change. Finally, if we can show that the ex-ante revenue increases, Lemma 16 implies that the payoff floor constraint is also satisfied for $t<s$.

Ex-ante revenue increases if

$$
\begin{gathered}
\left(e^{-r s}-e^{-r b}\right) \int_{w}^{v_{b}}\left(v-\frac{1-F(v)}{f(v)}\right) f(v) n(F(v))^{n-1} d v \\
+\left(e^{-r s^{\prime \prime}}-e^{-r b}\right) \int_{v_{b^{+}}}^{w}\left(v-\frac{1-F(v)}{f(v)}\right) f(v) n(F(v))^{n-1} d v \\
+\int_{v_{s^{\prime \prime}}}^{v_{b^{+}}}\left(e^{-r s^{\prime \prime}}-e^{-r T(v)}\right)\left(v-\frac{1-F(v)}{f(v)}\right) f(v) n(F(v))^{n-1} d v>0 .
\end{gathered}
$$

By subtracting the condition A.17 from the above inequality, we obtain:

$$
\left(e^{-r s}-e^{-r b}\right) \int_{w}^{v_{b}}\left(\frac{F(w)-1}{f(v)}\right) f(v) n(F(v))^{n-1} d v
$$




$$
\begin{gathered}
+\left(e^{-r s^{\prime \prime}}-e^{-r b}\right) \int_{v_{b^{+}}}^{w}\left(\frac{F(w)-1}{f(v)}\right) f(v) n(F(v))^{n-1} d v \\
+\int_{v_{s^{\prime \prime}}}^{v_{b^{+}}}\left(e^{-r s^{\prime \prime}}-e^{-r T(v)}\right)\left(\frac{F(w)-1}{f(v)}\right) f(v) n(F(v))^{n-1} d v>0 .
\end{gathered}
$$

This can be rearranged to

$$
\begin{gathered}
\left(e^{-r s}-e^{-r b}\right) \int_{w}^{v_{b}} n(F(v))^{n-1} d v+\left(e^{-r s^{\prime \prime}}-e^{-r b}\right) \int_{v_{b^{+}}}^{w} n(F(v))^{n-1} d v \\
+\int_{v_{s^{\prime \prime}}}^{v_{b^{+}}}\left(e^{-r s^{\prime \prime}}-e^{-r T(v)}\right) n(F(v))^{n-1} d v<0 .
\end{gathered}
$$

If we multiply the LHS by $J_{a}(w) f(w)$, we get

$$
\begin{aligned}
& \quad\left(e^{-r s}-e^{-r b}\right) \int_{w}^{v_{b}} J_{a}(w) f(w) n(F(v))^{n-1} d v \\
& +\left(e^{-r s^{\prime \prime}}-e^{-r b}\right) \int_{v_{b^{+}}}^{w} J_{a}(w) f(w) n(F(v))^{n-1} d v \\
& +\int_{v_{s^{\prime \prime}}}^{v_{b^{+}}}\left(e^{-r s^{\prime \prime}}-e^{-r T(v)}\right) J_{a}(w) f(w) n(F(v))^{n-1} d v \\
& <\quad\left(e^{-r s}-e^{-r b}\right) \int_{w}^{v_{b}} J_{a}(v) f(v) n(F(v))^{n-1} d v \\
& +\left(e^{-r s^{\prime \prime}}-e^{-r b}\right) \int_{v_{b^{+}}}^{w} J_{a}(v) f(v) n(F(v))^{n-1} d v \\
& +\int_{v_{s^{\prime \prime}}}^{v_{b^{+}}}\left(e^{-r s^{\prime \prime}}-e^{-r T(v)}\right) J_{a}(v) f(v) n(F(v))^{n-1} d v=0 .
\end{aligned}
$$

where the last equality is the condition for the unchanged payoff floor constraint at $a$.

Finally, we want to use the fact that $v(1-F(v))$ is concave on an interval $[0, \bar{v}]$. The following Lemma shows that a feasible solution cannot end with an atom of trade.

Lemma 21. Let $T$ be a feasible solution. Then for all $t>0$ such that $v_{t}>0$, there exists $w<v_{t}$ such that $T(v)>t$ for all $v \leq w$.

Proof. Suppose by contradiction that for some $t$ with $v_{t}>0$, we have $T(v)=t$ for all $v \in\left[0, v_{t}\right]$. Then for all $\varepsilon>0$ the payoff floor constraint at $t-\varepsilon$ is

$$
\int_{0}^{v_{t}} e^{-r \varepsilon} J_{t-\varepsilon}(v) d F_{t-\varepsilon}^{(n)}(v)+\int_{v_{t}}^{v_{t-\varepsilon}} e^{-r(T(v)-t)} J_{t-\varepsilon}(v) d F_{t-\varepsilon}^{(n)}(v) \geq \int_{v_{t}}^{v_{t-\varepsilon}} J_{t-\varepsilon}(v) d F_{t-\varepsilon}^{(n)}(v) .
$$

Rearranging this we get

$$
\int_{v_{t}}^{v_{t-\varepsilon}}\left(e^{-r(T(v)-t)}-1\right) J_{t-\varepsilon}(v) d F_{t-\varepsilon}^{(n)}(v) \geq\left(1-e^{-r \varepsilon}\right) \int_{0}^{v_{t}} J_{t-\varepsilon}(v) d F_{t-\varepsilon}^{(n)}(v),
$$


which is a contradiction because for $\varepsilon$ sufficiently small, the RHS is strictly positive but the LHS is non-positive.

Now we have all lemmas needed to complete the proofs of Theorems 2 and 3 .

Proof of Part (ii) of Theorem 2. Since $\phi>-1$, there exists a valuation $\bar{v}>0$ such that for all $v \in[0, \bar{v}],\left(f^{\prime}(v) v\right) / f(v)>-2$ which implies that $v(1-F(v))$ is concave on this interval. Lemma 21 shows that the optimal solution does not end with an atom. Therefore, Lemmas 19 and 20 imply that there exists a time $\bar{t}$ with $v_{\bar{t}} \leq \bar{v}$ after which the payoff floor must be binding for all $t$ at the optimal solution. Lemma 15 shows that this is not possible if $n>\bar{N}(F)$. Proposition 3 and the existence of an optimal solution (Proposition 1) therefore imply that the efficient auction is the only element in the feasible set of the auxiliary problem if $n>\bar{N}(F)$. This completes the proof of part (i) of Theorem 2 .

Proof of Theorem 3. By Lemmas 19 and 20 and Assumption A4, the payoff floor constraint must be binding at the optimal solution and by Lemma 15 the solution must satisfy (4.2).

Proof of Corollary 1. Part (i) is immediate. For part (ii) we use a Taylor expansion of $f(v)$ at zero to obtain

$$
\phi=\lim _{v \rightarrow 0} \frac{f^{\prime}(v) v}{f(v)}=\lim _{v \rightarrow 0} \frac{f^{\prime}(v) v}{f^{\prime}(0) v}=1 .
$$

\section{A.4 Proofs for Section 5}

To justify our auxiliary mechanism design approach, we need to show that the solution to our auxiliary problem is the limit of a sequence of equilibria in discrete time games. Again, we give a graphical overview (see Figure A.2 of the various lemmas that are used in Section 5 and this Appendix to develop the discrete time approximation.

\section{A.4.1 Construction of a Solution to the Generalized Payoff Floor Constraint}

The key step of the approximation is to discretize the solution to the binding payoff floor constraint. In order to do that, we first need to find a feasible solution such that the payoff floor constraint is strictly slack. We use a change of variables $y=\dot{v}_{t}$ to rewrite the ODE obtained in Lemma 12 as

$$
y^{\prime}(v)=-r-g(v, K) y(v)-h(v, K)(y(v))^{2} .
$$

Any solution to the above ODE with $K>1$ would lead to a strictly slack payoff floor constraint. Our goal is to show that the solution to the ODE exists and converges as $K \searrow 1$. For fixed $K$, existence and uniqueness would follow from Picard-Lindelof theorem if the RHS is locally Lipschitz 


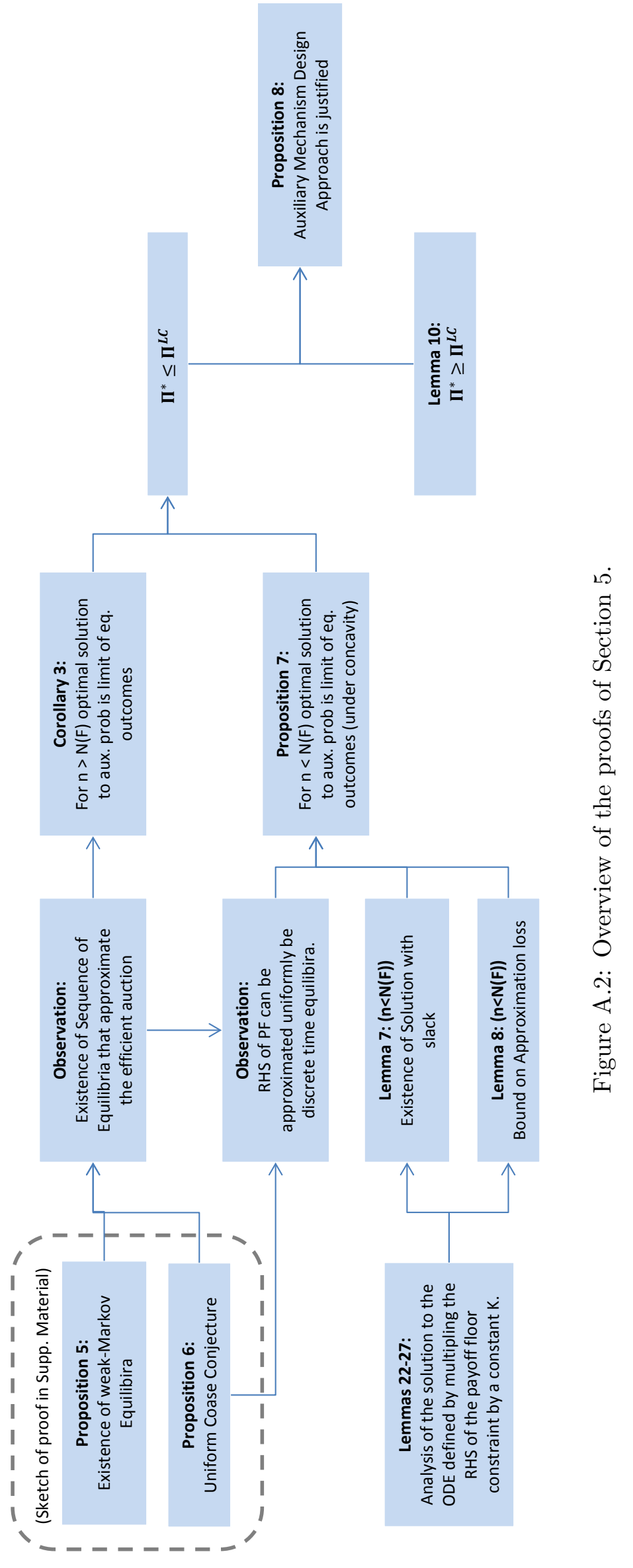


continuous in $y$ for all $v \geq 0$. Unfortunately, although the RHS is locally Lipschitz continuous for all $v>0$, its Lipschitz continuity may fail at $v=0$. Therefore, for $v$ strictly away from 0 , the standard argument applies given Lipschitz continuity, but for neighborhood around 0, we need a different argument. In what follows, we will center our analysis on the neighborhood of $v=0$. First, we show that the operator

$$
\left(L_{K} y\right)(v):=-r v-\int_{0}^{v} g(s, K) y(s)+h(s, K)(y(s))^{2} d s
$$

is a contraction mapping. Next, we show that the fixed point of $L_{K}$ converges uniformly to the fixed point of $L_{1}$ as $K \searrow 1$. Finally, we show that we can obtain a sequence of solutions $T^{K}$ that converge (pointwise) to the solution of the binding payoff floor constraint (with $K=1$ ) and show that the revenue of these solutions also converges to the value of the auxiliary problem.

Lemma 14 can be used to derive bounds for the RHS of (A.18). We treat the cases of $\kappa \in(-1,0]$ and $\kappa>0$ separately. Moreover, when $\kappa>0$, we restrict attention to the case of $\kappa \in(0,1 / r)$. Since by Proposition 2 the solution to the auxiliary problem is invariant with respect to $r$, our analysis for $\kappa<1 / r$ carries over to the case of $\kappa \geq 1 / r$.

Lemma 22. (i) Suppose $\kappa \in(-1,0]$. Then there exists $\hat{\kappa} \in(0,1), \Gamma>1, \gamma>0$, and $\xi>r /(1-\hat{\kappa})$ such that

$$
\sup _{K \in[1, \Gamma], v \in(0, \gamma]}|g(v, K)| v+2 \xi h(v, K) v^{2}<\hat{\kappa} .
$$

(ii) Suppose $\kappa \in(0,1 / r)$. Then there exists $0<\underline{\kappa}<\kappa<\bar{\kappa}<1 / r, \Gamma>1, \gamma>0$, such that for all $K \in[1, \Gamma]$ and all $v \in(0, \gamma]$,

$$
\underline{\kappa}<|g(v, K)| v+\frac{2}{\bar{\kappa}} h(v, K) v^{2}<\bar{\kappa},
$$

and

$$
h(v, K)\left(\frac{v}{\bar{\kappa}}\right)^{2}<\frac{1}{\bar{\kappa}}-r .
$$

Proof. For part (i), first note that since $\kappa \in(-1,0]$, A.7) implies the existence of $\hat{\kappa} \in(0,1), \tilde{\Gamma}>1$, and $\gamma>0$ such that

$$
\sup _{K \in[1, \tilde{\Gamma}], v \in(0, \gamma]}|g(v, K)| v<\hat{\kappa} .
$$

Next, fix an arbitrary $\xi>r /(1-\hat{\kappa})$. Then A.8) implies that we have, for $\Gamma<\tilde{\Gamma}$ sufficiently close to 1 ,

$$
\sup _{K \in[1, \Gamma], v \in(0, \gamma]} 2 \xi h(v, K) v^{2}<\hat{\kappa}-\sup _{K \in[1, \tilde{\Gamma}], v \in(0, \gamma]}|g(v, K)| v
$$


Hence

$$
\sup _{K \in[1, \Gamma], v \in(0, \gamma]}|g(v, K)| v+2 \xi h(v, K) v^{2} \leq \sup _{K \in[1, \tilde{\Gamma}], v \in(0, \gamma]}|g(v, K)| v+\sup _{K \in[1, \Gamma], v \in(0, \gamma]} 2 \xi h(v, K) v^{2}<\hat{\kappa} .
$$

Part (ii) can be proved analogously.

The next step is to define a space on which $L_{K}$ is a contraction mapping. For $\kappa \in(-1,0]$ we use the space

$$
\mathcal{Y}_{\xi}:=\{y:[0, \gamma] \rightarrow \mathbb{R} \mid y(v) \in[-\xi v, \xi v]\}
$$

equipped with the norm

$$
\|y\|:=\sup _{v \in(0, \gamma]} \frac{|y(s)|}{s} .
$$

For $\kappa \in(0,1 / r)$ we use the space

$$
\mathcal{Z}_{\bar{\kappa}}:=\left\{y:[0, \gamma] \rightarrow \mathbb{R} \mid y(v) \in\left[-\frac{1}{\bar{\kappa}} v, 0\right]\right\}
$$

and equip it with the norm

$$
\|y\|_{\bar{\kappa}}=\sup _{s \in(0, \gamma]} \frac{|y(s)|}{s^{\bar{\kappa}+1}} .
$$

Lemma 23. The spaces $\left(\mathcal{Y}_{\xi},\|\cdot\|\right)$ and $\left(\mathcal{Z}_{\bar{\kappa}},\|\cdot\|_{\bar{\kappa}}\right)$ are Banach spaces.

Proof. It is clear that $\|\cdot\|$ is a norm: (1) $\|y\| \geq 0,(2)\|y\|=0$ implies $|y(v)| / v=0$ for all $v \in(0, \gamma]$ so $y \equiv 0$, and $(3)\|y+z\|=\sup _{v \in(0, \gamma]}|y(v)+z(v)| / v \leq \sup _{v \in(0, \gamma]}(|y(v)|+|z(v)|) / v$ $=\sup _{v \in(0, \gamma]}|y(v)| / v+|z(v)| / v \leq \sup _{v \in(0, \gamma]}|y(v)| / v+\sup _{v \in(0, \gamma]}|z(v)| / v=\|y\|+\|z\|$. To see that $\left(\mathcal{Y}_{\xi},\|\cdot\|\right)$ is complete, note that it is isomorphic to the space $\{x:[0, \gamma] \rightarrow \mathbb{R} \mid x(v) \in[-\xi, \xi]\}$ with the standard supremum norm (via the mapping $x(s)=y(s) s)$. Since this space is complete, the original space is also complete.

One can verify that $\|\cdot\|_{\bar{\kappa}}$ is a norm by the same argument as for $\|\cdot\|$. Next one can also show that $\mathcal{Z}_{\bar{\kappa}}$ equipped with the norm $\|\cdot\|$ is complete by the same argument as for $\left(\mathcal{Y}_{\xi},\|\cdot\|\right)$. To show the completeness of $\left(\mathcal{Z}_{\bar{\kappa}},\|\cdot\|_{\bar{\kappa}}\right)$, let $\left(y_{m}\right) \in \mathcal{Z}_{\bar{\kappa}}$ be a $\|\cdot\|_{\bar{\kappa}}$-Cauchy sequence with limit $y^{*}:[0, \gamma] \rightarrow \mathbb{R}$. Since $1 / s^{\bar{\kappa}+1}>1 / s$ for $s \in(0,1),\|\cdot\|_{\bar{\kappa}} \geq\|\cdot\|$. Hence $\left(y_{m}\right)$ is also a $\|\cdot\|$-Cauchy sequence and $y_{m} \rightarrow y^{*}$ (w.r.t. to $\left.\|\cdot\|\right)$. It follows from the completeness of $\left(\mathcal{Z}_{\bar{\kappa}},\|\cdot\|\right)$ that $y^{*} \in \mathcal{Z}_{\bar{\kappa}}$. Therefore, $\left(\mathcal{Z}_{\bar{\kappa}},\|\cdot\|_{\bar{\kappa}}\right)$ is complete.

Now we are ready to show that $L_{K}$ is a contraction mapping.

Lemma 24. (i) Suppose $\kappa \in(-1,0]$. If $\hat{\kappa}, \Gamma, \gamma$, and $\xi>r /(1-\hat{\kappa})$ satisfy A.19), then $L_{K} \mathcal{Y}_{\xi} \subset \mathcal{Y}_{\xi}$ and $L_{K}$ is a contraction mapping on $\left(\mathcal{Y}_{\xi},\|\cdot\|\right)$.

(ii) Suppose $\kappa \in(0,1 / r)$. If $0<\underline{\kappa}<\kappa<\bar{\kappa}<1 / r, \Gamma>1$, and $\gamma>0$, satisfy A.20 and A.21, then $L_{K} \mathcal{Z}_{\bar{\kappa}} \subset \mathcal{Z}_{\bar{\kappa}}$ and $L_{K}$ is a contraction mapping on $\left(\mathcal{Z}_{\bar{\kappa}},\|\cdot\|_{\bar{\kappa}}\right)$. 
Proof. (i) We first show that $L_{K} \mathcal{Y}_{\xi} \subset \mathcal{Y}_{\xi}$. For all $y \in \mathcal{Y}_{\xi}$ we have

$$
\begin{aligned}
\left|\left(L_{K} y\right)(v)\right| & =\left|-r v-\int_{0}^{v} g(s, K) y(s)+h(s, K)(y(s))^{2} d s\right| \\
& \leq r v+\int_{0}^{v}|g(s, K)||y(s)|+h(s, K)(y(s))^{2} d s \\
& \leq r v+\int_{0}^{v}|g(s, K)| \xi s+h(s, K)(\xi s)^{2} d s \\
& \leq r v+\xi \hat{\kappa} v \\
& \leq \xi v,
\end{aligned}
$$

where we have used $y \in \mathcal{Y}_{\xi}$ for the second inequality, A.19) for the third inequality, and $\xi>r /(1-\hat{\kappa})$ for the last inequality. Next, suppose $y, z \in \mathcal{Y}_{\xi}$. We have

$$
\begin{aligned}
\left\|L_{K} z-L_{K} y\right\| & =\sup _{v \in(0, \gamma]} \frac{1}{v}\left|\int_{0}^{v} g(s, K)(z(s)-y(s))+h(s, K)\left((z(s))^{2}-(y(s))^{2}\right) d s\right| \\
& \leq \sup _{v \in(0, \gamma]} \frac{1}{v} \int_{0}^{v}|g(s, K)||z(s)-y(s)|+h(s, K)|z(s)-y(s)||z(s)+y(s)| d s \\
& \leq \sup _{v \in(0, \gamma]} \frac{1}{v} \int_{0}^{v}|g(s, K)|\|z-y\| s+h(s, K)\|z-y\| s 2 \xi s d s \\
& \leq\|z-y\| \sup _{v \in(0, \gamma]} \frac{1}{v} \int_{0}^{v}|g(s, K)| s+2 \xi h(s, K) s^{2} d s \\
& \leq\|z-y\| \sup _{v \in(0, \gamma]} \frac{1}{v} \int_{0}^{v} \hat{\kappa} d s \\
& =\hat{\kappa}\|z-y\| .
\end{aligned}
$$

For the first inequality, we have used that $y, z \in \mathcal{Y}_{\xi}$ and for the last inequality we have used (A.19). Since $\hat{\kappa} \in(0,1), L_{K}$ is a contraction mapping on $\left(\mathcal{Y}_{\xi},\|\cdot\|\right)$.

(ii) For $z \in \mathcal{Z}_{\bar{\kappa}}$, we have

$$
\begin{aligned}
\left(L_{K} z\right)(v) & =-r v-\int_{0}^{v} g(s, K) z(s)+h(s, K)(z(s))^{2} d s \\
& \geq-r v-\int_{0}^{v} h(s, K)\left(\frac{s}{\bar{\kappa}}\right)^{2} d s \\
& \geq-r v-\int_{0}^{v} \frac{1}{\bar{\kappa}}-r d s \\
& =-\frac{1}{\bar{\kappa}} v
\end{aligned}
$$

and

$$
\left(L_{K} z\right)(v)=-r v-\int_{0}^{v} g(s, K) z(s)+h(s, K)(z(s))^{2} d s
$$




$$
\begin{aligned}
& \leq-r v+\int_{0}^{v} g(s, K) \frac{1}{\bar{\kappa}} s d s \\
& \leq-r v+\frac{1}{\bar{\kappa}} \int_{0}^{v} \bar{\kappa} d s \\
& =0 .
\end{aligned}
$$

Therefore, $L_{K} \mathcal{Z}_{\bar{\kappa}} \subset \mathcal{Z}_{\bar{\kappa}}$. For $y, z \in \mathcal{Z}_{\bar{\kappa}}$ we have

$$
\begin{aligned}
\left\|L_{K} z-L_{K} y\right\| & =\sup _{v \in(0, \gamma]} \frac{1}{v^{\bar{\kappa}+1}}\left|\int_{0}^{v} g(s, K)(z(s)-y(s))+h(s, K)\left((z(s))^{2}-(y(s))^{2}\right) d s\right| \\
& \leq \sup _{v \in(0, \gamma]} \frac{1}{v^{\bar{\kappa}+1}} \int_{0}^{v}|g(s, K)||z(s)-y(s)|+h(s, K)|z(s)-y(s)||z(s)+y(s)| d s \\
& \leq \sup _{v \in(0, \gamma]} \frac{1}{v^{\bar{\kappa}+1}} \int_{0}^{v}|g(s, K)|\|z-y\| s^{\bar{\kappa}+1}+h(s, K)\|z-y\| s^{\bar{\kappa}+1} \frac{2}{\bar{\kappa}} s d s \\
& =\sup _{v \in(0, \gamma]} \frac{1}{v^{\bar{\kappa}+1}}\|z-y\| \int_{0}^{v} s^{\bar{\kappa}}\left(|g(s, K)| s+h(s, K) s^{2} \frac{2}{\bar{\kappa}}\right) d s \\
& \leq\|z-y\| \sup _{v \in(0, \gamma]} \frac{1}{v^{\bar{\kappa}+1}} \int_{0}^{v} \bar{\kappa} s^{\bar{\kappa}} d s \\
& \leq\|z-y\| \sup _{v \in(0, \gamma]} \frac{\bar{\kappa}}{\bar{\kappa}+1} \\
& =\frac{\bar{\kappa}}{\bar{\kappa}+1}\|z-y\| .
\end{aligned}
$$

Therefore, $L_{K}$ is a contraction mapping on $\left(\mathcal{Z}_{\bar{\kappa}},\|\cdot\|_{\bar{\kappa}}\right)$.

Note that the solution for $K=1$ (see 4.2)

$$
y(v)=-\int_{0}^{v} r e^{-\int_{w}^{v} g(x) d x} d w
$$

satisfies

$$
\lim _{v \rightarrow 0} y^{\prime}(v)=\lim _{v \rightarrow 0}\left(-r+g(v) \int_{0}^{v} r e^{-\int_{w}^{v} g(x) d x} d w\right)=-r .
$$

Therefore, $y \in \mathcal{Y}_{\xi}$ if $\kappa \in(-1,0]$ and $y \in \mathcal{Z}_{\bar{\kappa}}$ if $\kappa \in(0,1 / r)$.

We also need to show that the fixed-point of $L_{K}$ is non-positive as required by the condition $\dot{v}_{t} \leq 0$. For $\kappa \in(0,1 / r)$, this follows immediately since $y \in \mathcal{Z}_{\bar{\kappa}}$ implies $y(v) \leq 0$. For the other case, we have the following lemma.

Lemma 25. If $\kappa \in(-1,0]$, then the fixed-point of $L_{K}$ satisfies $y(v)<0$ for all $v \leq \gamma$.

Proof. We use the Picard-Lindelof iteration starting with the constant function $y^{0}(v)=0$. We need to distinguish two subcases:

(i) $\kappa<0$. Since $\kappa=\lim _{v \rightarrow 0} v g(v)$ and $g(v)=\lim _{K \rightarrow 1} g(v, K)$, there exist $\gamma>0, \Gamma>1$ such that $g(v, K)<0$ for all $v \in(0, \gamma]$ and $K \in[1, \Gamma]$. We argue by induction that $L_{K}^{m} 0<0$ for all $n$. 
For $m=1$ we have $\left(L_{K}^{1} 0\right)(v)=-r v<0$. For $n>1$ we have

$$
\begin{aligned}
\left(L_{K}^{m} 0\right)(v) & =-r v-\int_{0}^{v} g(s, K)\left(L_{K}^{m-1} 0\right)(s)+h(s, K)\left(\left(L_{K}^{m-1} 0\right)(s)\right)^{2} d s \\
& \leq-r v-\int_{0}^{v} g(s, K)\left(L_{K}^{m-1} 0\right)(s) d s \\
& \leq-r v
\end{aligned}
$$

where the first inequality follows from that fact that $h>0$, and the second inequality follows from the induction assumption $\left(L_{K}^{m-1} 0\right)(s) \leq 0$ and the fact that $g(s, K)<0$. Therefore, the fixed point on $\mathcal{Y}_{\xi}$ must be negative.

(ii) $\kappa=0$. In this case, $g(v, K) v \rightarrow 0$ as $K \searrow 1$. Therefore, for all $\varepsilon>0$, there exist $\gamma>0, \Gamma>1$ such that for all $v \in(0, \gamma]$ and $K \in[1, \Gamma]$,

$$
g(v, K) v \pm \frac{1}{1-\varepsilon} h(v, K) v^{2} \in[-\varepsilon, \varepsilon]
$$

Fix $\varepsilon<1 / 2$. We now show inductively that $-\frac{1-\varepsilon^{m}}{1-\varepsilon} r v \leq\left(L_{K}^{m} 0\right)(v) \leq 0$. This is clearly true for $n=1$. For $m>1$, we have

$$
\begin{aligned}
\left(L_{K}^{m+1} 0\right)(v) & =-r v-\int_{0}^{v} g(s, K)\left(L_{K}^{m} 0\right)(s)+h(s, K)\left(\left(L_{K}^{m} 0\right)(s)\right)^{2} d s \\
& \geq-r v-\int_{0}^{v}|g(s, K)|\left|\left(L_{K}^{m} 0\right)(s)\right|+h(s, K)\left(\frac{1-\varepsilon^{m}}{1-\varepsilon} r s\right)^{2} d s \\
& \geq-r v-\int_{0}^{v}|g(s, K)| \frac{1-\varepsilon^{m}}{1-\varepsilon} r s+h(s, K)\left(\frac{1-\varepsilon^{m}}{1-\varepsilon} r s\right)^{2} d s \\
& \geq-r v-\frac{1-\varepsilon^{m}}{1-\varepsilon} r \int_{0}^{v}|g(s, K)| s+\frac{1-\varepsilon^{m}}{1-\varepsilon} r h(s, K) s^{2} d s \\
& \geq-r v-\frac{1-\varepsilon^{m}}{1-\varepsilon} r \varepsilon \\
& =-\frac{1-\varepsilon^{m+1}}{1-\varepsilon} r v
\end{aligned}
$$

where the first inequality follows from $\left(L_{K}^{m} 0\right)(v) \leq 0$ and the third inequality follows from induction assumption for $n$. Moreover,

$$
\begin{aligned}
\left(L_{K}^{m+1} 0\right)(v) & =-r v-\int_{0}^{v} g(s, K)\left(L_{K}^{m} 0\right)(s)+h(s, K)\left(\left(L_{K}^{m} 0\right)(s)\right)^{2} d s \\
& \leq-r v-\int_{0}^{v} g(s, K)\left(L_{K}^{m} 0\right)(s) d s \\
& \leq-r v+\int_{0}^{v}|g(s, K)| \frac{1-\varepsilon^{m}}{1-\varepsilon} r s d s \\
& \leq-r v+\frac{1-\varepsilon^{m}}{1-\varepsilon} \varepsilon r v
\end{aligned}
$$




$$
<0
$$

where the last inequality holds for $\varepsilon<1 / 2$. Therefore, $-\frac{1-\varepsilon^{m+1}}{1-\varepsilon} r v \leq\left(L_{K}^{m+1} 0\right)(v)<0$. Hence, as in the previous two cases, the fixed point on $\mathcal{Y}_{\xi}$ must be negative.

Let $y_{K}$ be the fixed point of $L_{K}$. Next we show that the fixed-point $y_{K}$ is continuous in $K$.

Lemma 26. (i) Suppose $\kappa \in(-1,0]$. For any $\varepsilon>0$, there exists $\Gamma>1$ and $\gamma>0$ such that $\left\|L_{K} y-L_{1} y\right\|<\varepsilon$ for all $K \in[1, \Gamma], v \in(0, \gamma]$ and all $y \in \mathcal{Y}_{\xi}$.

(i) Suppose $\kappa \in(0,1 / r)$. For any $\varepsilon>0$, there exists $\Gamma>1$ and $\gamma>0$ such that $\left\|L_{K} y-L_{1} y\right\|<\varepsilon$ for all $K \in[1, \Gamma], v \in(0, \gamma]$ and all $y \in \mathcal{Z}_{\bar{\kappa}}$.

Proof. (i) By Lemma 22, for any $\varepsilon>0$, there exists $\Gamma>1$ such that

$$
\sup _{v \in(0, \gamma]}|g(v, K) v-g(v, 1) v|<\frac{\varepsilon}{\xi(1+\xi)}
$$

and

$$
\sup _{v \in(0, \gamma]}\left|h(v, K) v^{2}-h(v, 1) v^{2}\right|<\frac{\varepsilon}{\xi(1+\xi)},
$$

for all $K \in[1, \Gamma]$. Then, for any $y \in \mathcal{Y}_{\xi}$,

$$
\begin{aligned}
\left\|L_{K} y-L_{1} y\right\| & =\sup _{v \in(0, \gamma]} \frac{\left|\int_{0}^{v}(g(s, K)-g(s, 1)) y(s)+(h(s, K)-h(s, 1))(y(s))^{2} d s\right|}{v} \\
& \leq \sup _{v \in(0, \gamma]} \frac{\left|\int_{0}^{v}(g(s, K)-g(s, 1))\|y\| s d s\right|+\left|\int_{0}^{v}(h(s, K)-h(s, 1))\|y\|^{2} s^{2} d s\right|}{v} \\
& \leq \sup _{v \in(0, \gamma]} \frac{\|y\| \frac{\varepsilon}{\xi(1+\xi)} v+\|y\|^{2} \frac{\varepsilon}{\xi(1+\xi)} v}{v} \\
& =\frac{\varepsilon}{\xi(1+\xi)}\|y\|(1+\|y\|) \\
& \leq \varepsilon
\end{aligned}
$$

This proves part (i). The proof for (ii) is similar.

Therefore, $y_{K}=\lim _{n \rightarrow \infty}\left(L_{K}\right)^{m} y$ for any $y$ in $\left(\mathcal{Y}_{\xi},\|\cdot\|\right)$. That is, for any fixed $\varepsilon>0$, for any $y \in \mathcal{Y}_{\xi}$, there exists $\Gamma>1$ such that, for all $K \in[1, \Gamma],\left\|\left(L_{K}\right)^{m} y-y_{K}\right\|<\varepsilon$ for sufficiently large $m$. This result is then used to show $y_{K}(v) \rightarrow y_{1}(v)$ uniformly in the neighborhood of $v=0$ as $K \rightarrow 1$.

Lemma 27. There exist $\gamma>0$ such that $\sup _{v \in(0, \gamma]}\left(y_{K}(v)-y_{1}(v)\right) \rightarrow 0$ as $K \rightarrow 1$.

Proof. Again we distinguish two cases. Consider first the case of $\kappa \in(-1,0]$. In this case, it suffices to show that $\left\|y_{K}-y_{1}\right\| \rightarrow 0$ since convergence in the norm $\|\cdot\|$ implies uniform convergence. The 
proof here is taken from Lee and Liu (2013, Lemma 13(b)). For completeness, we replicate it here. Fix any $K \in[1, \Gamma]$, where $\Gamma$ is given by Lemma 26 . For any $m>1$,

$$
\begin{aligned}
\left\|\left(L_{K}\right)^{m} y-\left(L_{1}\right)^{m} y\right\| & =\left\|\left(L_{K}\right)^{m} y-L_{1}\left(L_{K}\right)^{m-1} y+L_{1}\left(L_{K}\right)^{m-1} y-\left(L_{1}\right)^{m} y\right\| \\
& =\left\|L_{K}\left(L_{K}\right)^{m-1} y-L_{1}\left(L_{K}\right)^{m-1} y+L_{1}\left(L_{K}\right)^{m-1} y-L_{1}\left(L_{1}\right)^{m-1} y\right\| \\
& \leq\left\|L_{K}\left(L_{K}\right)^{m-1} y-L_{1}\left(L_{K}\right)^{m-1} y\right\|+\left\|L_{1}\left(L_{K}\right)^{m-1} y-L_{1}\left(L_{1}\right)^{m-1} y\right\| \\
& \leq \varepsilon+\lambda\left\|\left(L_{K}\right)^{m-1} y-\left(L_{1}\right)^{m-1} y\right\|,
\end{aligned}
$$

where $\lambda<1$ is the Lipschitz constant of the contraction mapping $L_{1}$ and the last inequality follows from Lemma 26. Inductively, for any $m>1$,

$$
\begin{aligned}
\left\|\left(L_{K}\right)^{m} y-\left(L_{1}\right)^{m} y\right\| & \leq \varepsilon+\lambda\left\|\left(L_{K}\right)^{m-1} y-\left(L_{1}\right)^{m-1} y\right\| \\
& \leq \varepsilon+\lambda \varepsilon+\lambda^{2} \varepsilon+\cdots+\lambda^{m-1}\left\|L_{K} y-L_{1} y\right\| \\
& \leq \varepsilon+\lambda \varepsilon+\lambda^{2} \varepsilon+\cdots+\lambda^{m-1} \varepsilon \\
& \leq \frac{\varepsilon}{1-\lambda},
\end{aligned}
$$

where the last inequality again follows from Lemma 26. Then

$$
\begin{aligned}
\left\|y_{K}-y_{1}\right\| & =\left\|y_{K}-\left(L_{K}\right)^{m} y+\left(L_{K}\right)^{m} y-\left(L_{1}\right)^{m} y+\left(L_{1}\right)^{m} y-y_{1}\right\| \\
& \leq\left\|y_{K}-\left(L_{K}\right)^{m} y\right\|+\left\|\left(L_{K}\right)^{m} y-\left(L_{1}\right)^{m} y\right\|+\left\|\left(L_{1}\right)^{m} y-y_{1}\right\| \\
& \leq\left\|y_{K}-\left(L_{K}\right)^{m} y\right\|+\left\|\left(L_{K}\right)^{m} y-\left(L_{1}\right)^{m} y\right\|+\left\|\left(L_{1}\right)^{m} y-y_{1}\right\| \\
& \leq \varepsilon+\frac{\varepsilon}{1-\lambda}+\varepsilon \\
& \leq \varepsilon \frac{3-2 \lambda}{1-\lambda} .
\end{aligned}
$$

Therefore, $\left\|y_{K}-y_{1}\right\| \rightarrow 0$ as $K \rightarrow 1$. The same proof applies to the case of $\kappa \in(0,1 / r)$.

Now we have all the ingredients necessary to prove Lemma 8

Proof of Lemma 8. The uniform convergence of $y_{K}$ implies that the cutoff sequence $v_{t}^{K}$ given by $v(t)=v(0)+\int_{0}^{t} y_{K}(v(s)) d s$ converges pointwise to the cutoff sequence $v_{t}=v_{t}^{1}$ associated with the trading time function $T(v)=T^{1}(v)$. Since $v_{t}$ is continuous and strictly decreasing (be Lemma 13), this implies that the trading time function

$$
T^{K}(v)=\sup \left\{t: v_{t}^{K} \geq v\right\}
$$

converges pointwise to $T(v)$. To see this, note that $\sup \left\{t: v_{t} \geq v\right\}=\sup \left\{t: v_{t}>v\right\}$, since $v_{t}$ is continuous and strictly decreasing. Now, for all $t$ such that $v_{t}>v$, there exists $K^{t}$ such that $v_{t}^{K}>v$ 
for all $K<K^{t}$. Hence,

$$
\lim _{K \searrow 1} \sup \left\{t: v_{t}^{K} \geq v\right\} \geq \sup \left\{t: v_{t}>v\right\}
$$

Similarly, for all $t$ such that $v_{t}<v$, there exists $K^{t}$ such that $v_{t}^{K}<v$ for $K<K^{t}$. Hence,

$$
\lim _{K \searrow 1} \sup \left\{t: v_{t}^{K} \geq v\right\} \leq \sup \left\{t: v_{t} \geq v\right\}
$$

Therefore, for all $v$, we have

$$
\lim _{K \searrow 1} \sup \left\{t: v_{t}^{K} \geq v\right\}=\sup \left\{t: v_{t} \geq v\right\}
$$

or equivalently,

$$
\lim _{K \searrow 1} T^{K}(v)=T(v)
$$

It remains to show that the seller's ex ante revenue converges. Notice that the sequence $e^{-r T^{K}(v)}$ is uniformly bounded by 1 . Therefore, the dominated convergence theorem implies that

$$
\lim _{K \searrow 1} \int_{0}^{1} e^{-r T^{K}(x)} J(x) d F^{(n)}(x)=\int_{0}^{1} e^{-r T(x)} J(x) d F^{(n)}(x) .
$$

\section{A.4.2 Discretization}

Proof of Lemma 9. For $t \in\{0, \Delta, 2 \Delta, \ldots\}$, define

$$
\tilde{v}_{t}^{K, \Delta}=\inf \left\{v \mid J\left(v \mid v \leq v_{t}^{K, \Delta}\right) \geq 0\right\}
$$

Consider the LHS of the payoff floor constraint at $t=k \Delta, k \in \mathbb{N}_{0}$. Notice that, for $k>0$, the new posterior at this point in time is equal to the old posterior at $((k-1) \Delta)^{+}$. Therefore, we can approximate the LHS of the payoff floor at $t=k \Delta$ as:

$$
\begin{aligned}
& \int_{0}^{v_{k \Delta}^{K, \Delta}} e^{-r\left(T^{K, \Delta}(v)-k \Delta\right)} J\left(v \mid v \leq v_{k \Delta}^{K, \Delta}\right) f^{(n)}\left(v \mid v \leq v_{k \Delta}^{K, \Delta}\right) d v \\
= & \int_{0}^{v_{k \Delta}^{K, \Delta}} e^{-r\left(T^{K}(v)-(k-1) \Delta\right)} e^{-r\left(T^{K, \Delta}(v)-T^{K}(v)-\Delta\right)} J\left(v \mid v \leq v_{k \Delta}^{K, \Delta}\right) f^{(n)}\left(v \mid v \leq v_{k \Delta}^{K, \Delta}\right) d v \\
= & \int_{\tilde{v}_{k \Delta}^{K, \Delta}}^{v_{k \Delta}^{K, \Delta}} e^{-r\left(T^{K}(v)-(k-1) \Delta\right)} e^{-r\left(T^{K, \Delta}(v)-T^{K}(v)-\Delta\right)} J\left(v \mid v \leq v_{k \Delta}^{K, \Delta}\right) f^{(n)}\left(v \mid v \leq v_{k \Delta}^{K, \Delta}\right) d v \\
& +\int_{0}^{\tilde{v}_{k \Delta}^{K, \Delta}} e^{-r\left(T^{K}(v)-(k-1) \Delta\right)} e^{-r\left(T^{K, \Delta}(v)-T^{K}(v)-\Delta\right)} J\left(v \mid v \leq v_{k \Delta}^{K, \Delta}\right) f^{(n)}\left(v \mid v \leq v_{k \Delta}^{K, \Delta}\right) d v
\end{aligned}
$$




$$
\begin{aligned}
\geq & \int_{\tilde{v}_{k \Delta}^{K, \Delta}}^{v_{k \Delta}^{K, \Delta}} e^{-r\left(T^{K}(v)-(k-1) \Delta\right)} J\left(v \mid v \leq v_{k \Delta}^{K, \Delta}\right) f^{(n)}\left(v \mid v \leq v_{k \Delta}^{K, \Delta}\right) d v \\
& +\int_{0}^{\tilde{v}_{k \Delta}^{K, \Delta}} e^{-r\left(T^{K}(v)-(k-1) \Delta\right)} e^{r \Delta} J\left(v \mid v \leq v_{k \Delta}^{K, \Delta}\right) f^{(n)}\left(v \mid v \leq v_{k \Delta}^{K, \Delta}\right) d v \\
\geq & \int_{\tilde{v}_{k \Delta}^{K, \Delta}}^{v_{k \Delta}^{K, \Delta}} e^{-r\left(T^{K}(v)-(k-1) \Delta\right)} J\left(v \mid v \leq v_{k \Delta}^{K, \Delta}\right) f^{(n)}\left(v \mid v \leq v_{k \Delta}^{K, \Delta}\right) d v \\
& +\int_{0}^{\tilde{v}_{k \Delta}^{K, \Delta}} e^{-r\left(T^{K}(v)-(k-1) \Delta\right)} J\left(v \mid v \leq v_{k \Delta}^{K, \Delta}\right) f^{(n)}\left(v \mid v \leq v_{k \Delta}^{K, \Delta}\right) d v \\
& -\int_{0}^{\tilde{v}_{k \Delta}^{K, \Delta}} e^{-r\left(T^{K}(v)-(k-1) \Delta\right)}\left(1-e^{r \Delta}\right) J\left(v \mid v \leq v_{k \Delta}^{K, \Delta}\right) f^{(n)}\left(v \mid v \leq v_{k \Delta}^{K, \Delta}\right) d v \\
= & \int_{0}^{v_{k \Delta}^{K, \Delta}} e^{-r\left(T^{K}(v)-(k-1) \Delta\right)} J\left(v \mid v \leq v_{k \Delta}^{K, \Delta}\right) f^{(n)}\left(v \mid v \leq v_{k \Delta}^{K, \Delta}\right) d v \\
& -\int_{0}^{\tilde{v}_{k \Delta}^{K, \Delta}} e^{-r\left(T^{K}(v)-(k-1) \Delta\right)}\left(1-e^{r \Delta}\right) J\left(v \mid v \leq v_{k \Delta}^{K, \Delta}\right) f^{(n)}\left(v \mid v \leq v_{k \Delta}^{K, \Delta}\right) d v .
\end{aligned}
$$

The first term in the last expression is equal to the LHS of the payoff floor constraints at $((k-1) \Delta)^{+}$ for the original solution $v^{K}$. Hence it is equal to $K \Pi^{E}\left(v_{k \Delta}^{K, \Delta}\right)$. Therefore, we have

$$
\begin{aligned}
& \int_{0}^{v_{k \Delta}^{K, \Delta}} e^{-r\left(T^{K, \Delta}(v)-k \Delta\right)} J\left(v \mid v \leq v_{k \Delta}^{K, \Delta}\right) f^{(n)}\left(v \mid v \leq v_{k \Delta}^{K, \Delta}\right) d v \\
= & K \Pi^{E}\left(v_{k \Delta}^{K, \Delta}\right)+\left(e^{r \Delta}-1\right) \int_{0}^{\tilde{v}_{k \Delta}^{K, \Delta}} e^{-r\left(T^{K}(v)-(k-1) \Delta\right)} J\left(v \mid v \leq v_{k \Delta}^{K, \Delta}\right) f^{(n)}\left(v \mid v \leq v_{k \Delta}^{K, \Delta}\right) d v \\
\geq & K \Pi^{E}\left(v_{k \Delta}^{K, \Delta}\right)+\left(e^{r \Delta}-1\right) \int_{0}^{\tilde{v}_{k \Delta}^{K, \Delta}} J\left(v \mid v \leq v_{k \Delta}^{K, \Delta}\right) f^{(n)}\left(v \mid v \leq v_{k \Delta}^{K, \Delta}\right) d v \\
= & K \Pi^{E}\left(v_{k \Delta}^{K, \Delta}\right)-\left(e^{r \Delta}-1\right)\left[\Pi^{M}\left(v_{k \Delta}^{K, \Delta}\right)-\Pi^{E}\left(v_{k \Delta}^{K, \Delta}\right)\right] \\
= & K \Pi^{E}\left(v_{k \Delta}^{K, \Delta}\right)-\left(e^{r \Delta}-1\right)\left[\frac{\Pi^{M}\left(v_{k \Delta}^{K, \Delta}\right)}{\Pi^{E}\left(v_{k \Delta}^{K, \Delta}\right)}-1\right] \Pi^{E}\left(v_{k \Delta}^{K, \Delta}\right) .
\end{aligned}
$$

Next we show that $\frac{\Pi^{M}\left(v_{k \Delta}^{K, \Delta}\right)}{\Pi^{E}\left(v_{k \Delta}^{K, \Delta}\right)}-1$ is uniformly bounded. Recall that by Assumption A4, there exist $0<M \leq 1 \leq L<\infty$ and $\alpha>0$ such that $M v^{\alpha} \leq F(v) \leq L v^{\alpha}$ for all $v \in[0,1]$. This implies that the rescaled truncated distribution

$$
\tilde{F}_{x}(v):=\frac{F(v x)}{F(x)}
$$

for all $v \in[0,1]$ is dominated by a function that is independent of $x$ :

$$
\tilde{F}_{x}(v) \leq \frac{L v^{\alpha} x^{\alpha}}{M x^{\alpha}}=\frac{L}{M} v^{\alpha}
$$

Next, we observe that the revenue of the efficient auction can be written in terms of the rescaled 
expected value of the second-highest order statistic of the rescaled distribution:

$$
\Pi^{E}(v)=\int_{0}^{1} v s \tilde{F}_{v}^{(n-1: n)}(s) d s
$$

If we define $\hat{F}(v):=\min \left\{1, \frac{L}{M} v^{\alpha}\right\}$ and $B:=\int_{0}^{1} s \hat{F}^{(n-1: n)}(s) d s$, then given $\tilde{F}_{x}(v) \leq \frac{L}{M} v^{\alpha}$ we can apply Theorem 4.4.1 in David and Nagaraja (2003) to obtain $\Pi^{E}(v) \geq B v>0$. Since $\Pi^{M}(v) \leq v$, we have

$$
\frac{\Pi^{M}\left(v_{k \Delta}^{K, \Delta}\right)}{\Pi^{E}\left(v_{k \Delta}^{K, \Delta}\right)}-1 \leq \frac{1}{B}-1 .
$$

Therefore, LHS of the payoff floor at $t=k \Delta$ is bounded below by

$$
\left[K-\left(e^{r \Delta}-1\right)\left(\frac{1}{B}-1\right)\right] \Pi^{E}\left(v_{k \Delta}^{K, \Delta}\right) .
$$

Clearly, for $\Delta$ sufficiently small, the term in the square bracket is greater than or equal to $(K+$ 1) $/ 2$.

Proof of Lemma 10. To see this, note that for $t \in(k \Delta,(k+1) \Delta]$ we have $v_{t}^{\Delta}=v_{(k+1) \Delta}^{\Delta}$ and hence

$$
\begin{aligned}
& \int_{0}^{v_{t}^{\Delta}} e^{-r\left(T^{\Delta}(v)-t\right)} J_{t}(v) n(F(v))^{n-1} f(v) d v \\
= & e^{-r((k+1) \Delta-t)} \int_{0}^{v_{(k+1) \Delta}^{\Delta}} e^{-r\left(T^{\Delta}(v)-(k+1) \Delta\right)} J_{(k+1) \Delta}(v) n(F(v))^{n-1} f(v) d v \\
\geq & e^{-r \Delta} \int_{0}^{v_{(k+1) \Delta}^{\Delta}} e^{-r\left(T^{\Delta}(v)-(k+1) \Delta\right)} J_{(k+1) \Delta}(v) n(F(v))^{n-1} f(v) d v \\
\geq & e^{-r \Delta} \Pi^{E}\left(v_{(k+1) \Delta}^{\Delta}\right) \\
= & e^{-r \Delta} \Pi^{E}\left(v_{t}^{\Delta}\right) \\
\geq & \Pi^{E}\left(v_{t}\right)-\varepsilon
\end{aligned}
$$

The last inequality holds if $\Delta$ is sufficiently small and Assumption A3 is satisfied. Hence $\Pi^{\Delta}(p, b) \leq$ $V(\Delta) \leq V_{\varepsilon}(0)$ for all $(p, b) \in \mathcal{E}(\Delta)$ if $\Delta<\Delta_{\varepsilon}$. 


\section{References}

Ausubel, L. M., P. Cramton, and R. J. Deneckere (2002): "Bargaining with Incomplete Information," in Handbook of Game Theory, ed. by R. J. Aumann, and S. Hart, vol. 3, chap. Ch. 50, pp. 1897-1945. Elsevier Science B.V.

Ausubel, L. M., and R. J. Deneckere (1989): "Reputation in Bargaining and Durable Goods Monopoly," Econometrica, 57(3), 511-531.

Bergemann, D., And M. SAid (2011): "Dynamic Auctions," in Wiley Encyclopedia of Operations Research and Management Science, ed. by J. J. Cochran. Wiley.

Bergin, J., and W. B. MacLeod (1993): "Continuous Time Repeated Games," International Economic Review, 34(1), 21-27.

Bester, H., and R. Strausz (2000): "Imperfect Commitment and the Revelation Principle: The Multi-Agent Case," Economics Letters, 69, 165-171.

- (2001): "Contracting with Imperfect Commitment and the Revelation Principle: The Single Agent Case," Econometrica, 69(4), 1007-1098.

Board, S., And A. Skrzypacz (2013): "Revenue Management with Forward-Looking Buyers," unpublished working paper.

Bulow, J. (1982): "Durable-Goods Monopolists," Journal of Political Economy, 90, 314-332.

Chen, C.-H. (2012): "Name Your own Price at Priceline.com: Strategic Bidding and Lockout Periods," Review of Economic Studies, 79(4), 1341-1369.

CoAse, R. H. (1972): "Durability and Monopoly," Journal of Law and Economics, 15(1), 143-149.

Courty, P., And H. Li (2000): "Sequential Screening," Review of Economic Studies, 67, 697-717.

David, H. A., And H. N. Nagaraja (2003): Order Statistics. Wiley-Interscience, 3rd edn.

Dewatripont, M. (1989): "Renegotiation and Information Revelation over Time: The Case of Optimal Labor Contracts," Quarterly Journal of Economics, 104(3), 589-619.

Dilme, F., And F. Li (2012): "Revenue Management Without Commitment: Dynamic Pricing and Periodic Fire Sales," working paper, University of Pennsylvania.

Eso, P., And B. Szentes (2007): "Optimal Information Disclosure in Auctions and the Handicap Auction," Review of Economic Studies, 74, 705-731. 
Freixas, X., R. Guesnerie, and J. Tirole (1985): "Planning under Incomplete Information and the Ratchet Effect," Review of Economic Studies, 52(2), 173-191.

Fuchs, W., And A. Skrzypacz (2010): "Bargaining with Arrival of New Traders," American Economic Review, 100, 802-836.

Fudenberg, D., D. Levine, and J. Tirole (1985): "Infinite-Horizon Models of Bargaining with One-Sided Incomplete Information," in Game-Theoretic Models of Bargaining, ed. by A. Roth, chap. 5, pp. 73-98. Cambridge University Press.

FudenberG, D., And J. Tirole (1991): Game Theory. MiT Press, Cambridge, Massachusetts.

Gershkov, A., And B. Moldovanu (2012): "Dynamic allocation and pricing: A mechanism design approach," International Journal of Industrial Organization, 30, 283-286.

Gul, F., H. Sonnenschein, and R. Wilson (1986): "Foundations of Dynamic Monopoly and the Coase Conjecture," Journal of Economic Theory, 39(1), 155-190.

Hart, O. D., And J. Tirole (1988): "Contract Renegotiation and Coasian Dynamics," Review of Economic Studies, 55(4), 509-540.

Hörner, J., And L. SAmuelson (2011): "Managing Strategic Buyers," Journal of Political Economy, 119(3), 379-425.

Krishna, V. (2002): Auction Theory. Academic Press.

Laffont, J.-J., And J. Tirole (1990): "Adverse Selection and Renegotiation in Procurement," Review of Economic Studies, 57(4), 597-625.

Lee, J., And Q. LiU (2013): "Gambling Reputation: Repeated Bargaining with Outside Options," Econometrica, 81(4), 1601-1672.

McAdams, D., and M. Schwarz (2007): "Credible Sales Mechanisms and Intermediaries," American Economic Review, 97(1), 260-276.

McAfee, R. P., And D. Vincent (1997): "Sequentially Optimal Auctions," Games and Economic Behavior, 18, 246-276.

Myerson, R. B. (1981): "Optimal Auction Design," Mathematics of Operations Reseach, 6, 58-63.

Pavan, A., I. Segal, And J. Tolkka (2013): "Dynamic Mechanism Design: A Myersonian Approach," Northwestern University, unpublished working paper.

Porter, R. H. (1995): "The Role of Information in U.S. Offshore Oil and Gas Lease Auction," Econometrica, 63(1), 1-27. 
Riley, J. G., and W. F. Samuelson (1981): "Optimal Auctions," American Economic Review, $71(3), 381-392$.

Skreta, V. (2006): "Sequentially Optimal Mechanisms," Review of Economic Studies, 73(4), 10851111.

_ (2011): "Optimal Auction Design under Non-Commitment," working paper, Stern School of Business.

Sobel, J. (1991): "Durable Goods Monopoly with Entry of New Consumers," Econometrica, 59(5), $1455-1485$.

Stokey, N. L. (1981): "Rational Expectations and Durable Goods Pricing," Bell Journal of Economics, 12(1), 112-128.

Strulovici, B. (2013): "Contract Renegotiation and the Coase Conjecture," unpublished manuscript, Northwestern University.

Vartiainen, H. (2013): "Auction Design without Commitment," Journal of the European Economic Association, 11, 316-342.

Wolitzky, A. (2010): "Dynamic monopoly with relational incentives," Theoretical Economics, 5, 479-518. 


\section{Supplemental Material}

\section{B Existence of Weak Markov Equilibria and the Uniform Coase Conjecture}

The proofs of Propositions 5 and 7 are very similar to the proofs in Ausubel and Deneckere (1989) (henceforth AD). Therefore, we adopt their notation and assume that the types of the bidders are i.i.d. draws from $U[0,1]$. We denote the type of buyer $i$ by $q^{i}$. The valuation for each type is given by the function $v(q):=F^{-1}(q)$. Note that $\mathbf{A} \mathbf{3}$ implies that the same condition also holds for $v$. In the following we will relax $\mathbf{A} 1$ and assume only that $F$ is continuous and strictly increasing (as in $\mathrm{AD}$ we could relax this even further to general distribution functions but this is not necessary for the purpose of the present paper) ${ }^{28}$ In the following subsection, we only state proofs for the parts of the proof of $\mathrm{AD}$ that need to be modified for the case of $n \geq 2$.

\section{B.1 Proof of Proposition 5}

In a weak-Markov equilibrium, the buyers' strategy can be described by a function $P:[0,1] \rightarrow[0,1]$. A bidder with type $q^{i}$ places a valid bid if and only if the announced reserve price is smaller than $P\left(q^{i}\right)$. Given that $v$ is strictly increasing, Lemma 1 implies that $P$ is non-decreasing.

Also by Lemma 1, the posterior of the seller at any history is described by the supremum of the support, which we denote by $q$. If all buyers play according to $P$, the seller's (unconditional) continuation profit for given $q$ i: 29

$$
R(q):=\max _{y \in[0, q]} \int_{y}^{q} v(z) d\left[n z^{n-1}-(n-1) z^{n}\right]+P(y) n(q-y) y^{n-1}+e^{-r \Delta} R(y)
$$

Let $Y(q)$ be the $\operatorname{argmax}$ correspondence and define $y(q):=\sup Y(q)$. Because the objective satisfies a single-crossing property, $Y(q)$ is increasing and hence single-valued almost everywhere. If $Y(q)$ is single-valued at $q$ the seller announces a reserve price $S(q)=P(y(q))$ if the posterior has upper bound $q$.

The buyers' indifference condition for the case that $Y(q)$ is single-valued so that the seller does not randomize, is given by:

$$
v(q)-P(q)=e^{-r \Delta}\left[v(q)-\frac{(y(q))^{n-1}}{q^{n-1}} S(q)-\frac{1}{q^{n-1}} \int_{y(q)}^{q} v(x) d x^{n-1}\right] .
$$

\footnotetext{
${ }^{28} \mathrm{In} \mathrm{AD}$ the valuation is decreasing in the type. We define $v$ to be increasing so that higher types have higher valuations.

${ }^{29}$ Dividing the RHS by $q^{n}$ and replacing $R(y)$ by $y^{n} R(y)$ would yield the conditional continuation profit. The unconditional version is more convenient for the subsequent development.
} 
If the seller randomizes over $Y(q)$ according to some probability measure $\mu$, then

$$
v(q)-P(q)=e^{-r \Delta}\left[v(q)-\int_{Y(q)}\left\{\frac{y^{n-1}}{q^{n-1}} P(y)+\frac{1}{q^{n-1}} \int_{y}^{q} v(x) d x^{n-1}\right\} d \mu(y)\right],
$$

which may require that $\mu$ depends on $P(q){ }^{30}$

We will be looking for left-continuous functions $R$ and $P$ such that (B.1) and (B.2) are satisfied 31 If this is true for all $q \in[0, \bar{q}]$, then we say that $(P, R)$ support a weak-Markov equilibrium on $[0, \bar{q}]$. The goal is to show the existence of a pair $(P, R)$ that supports a weak-Markov equilibrium on $[0,1]$. As in $\mathrm{AD}$, we can show that the seller's continuation profit is Lipschitz-continuous in $q$.

Lemma 28 (cf. Lemma A.2 in AD). If $(P, R)$ supports a weak-Markov equilibrium on $[0, \bar{q}]$, then $R$ is increasing and Lipschitz continuous satisfying

$$
0<R\left(q_{1}\right)-R\left(q_{2}\right) \leq n\left(q_{1}-q_{2}\right)
$$

for all $0 \leq q_{2}<q_{1} \leq \bar{q}$.

Proof. First, we show monotonicity:

$$
\begin{aligned}
R\left(q_{1}\right) & =\int_{y\left(q_{1}\right)}^{q_{1}} v(z) d\left[n z^{n-1}-(n-1) z^{n}\right]+P\left(y\left(q_{1}\right)\right) n\left(q_{1}-y\left(q_{1}\right)\right)\left(y\left(q_{1}\right)\right)^{n-1}+e^{-r \Delta} R\left(y\left(q_{1}\right)\right) \\
& \geq \int_{y\left(q_{2}\right)}^{q_{1}} v(z) d\left[n z^{n-1}-(n-1) z^{n}\right]+P\left(y\left(q_{2}\right)\right) n\left(q_{1}-y\left(q_{2}\right)\right)\left(y\left(q_{2}\right)\right)^{n-1}+e^{-r \Delta} R\left(y\left(q_{2}\right)\right) \\
& >\int_{y\left(q_{2}\right)}^{q_{2}} v(z) d\left[n z^{n-1}-(n-1) z^{n}\right]+P\left(y\left(q_{2}\right)\right) n\left(q_{2}-y\left(q_{2}\right)\right)\left(y\left(q_{2}\right)\right)^{n-1}+e^{-r \Delta} R\left(y\left(q_{2}\right)\right) \\
& =R\left(q_{2}\right)
\end{aligned}
$$

To show Lipschitz continuity, notice that the revenue from sales to types below $q_{2}$ in the continuation starting from $q_{1}$ is at most $R\left(q_{2}\right)$ and the revenue from types between $q_{2}$ and $q_{1}$ is bounded above by $P\left(q_{1}\right)\left(q_{1}^{n}-q_{2}^{n}\right) \stackrel{32}{32}$ Hence

$$
\begin{aligned}
R\left(q_{1}\right)-R\left(q_{2}\right) & \leq P\left(q_{1}\right)\left(q_{1}^{n}-q_{2}^{n}\right) \\
& \leq\left(q_{1}^{n}-q_{2}^{n}\right) \\
& \leq n\left(q_{1}-q_{2}\right)
\end{aligned}
$$

\footnotetext{
${ }^{30}$ In the following, we give details for the case that the seller does not randomize and refer to AD for the discussion of randomization by the seller.

${ }^{31}$ Left-continuity will be used in the proof of Proposition 7 in the next section.

${ }^{32}$ Suppose by contradiction that for the posterior $\left[0, q_{1}\right]$, the expected payment that the seller can extract from some type $q \in\left[q_{2}, q_{1}\right]$ is greater or equal than $P\left(q_{1}\right)$. In order to arrive at a history where the posterior is [0, $\left.q_{1}\right]$, the seller must have used reserve price $P\left(q_{1}\right)$ in the previous period. But then all types in $\left[q, q_{1}\right]$ would prefer to bid in the previous period because they expect to make higher payments if they wait. This is a contradiction.
} 
Using this Lemma, we can show that an existence result for $[0, \bar{q}]$ can be extended to the whole interval $[0,1]$.

Lemma 29 (cf. Lemma A.3 in $\mathrm{AD})$. Suppose $\left(P_{\bar{q}}, R_{\bar{q}}\right)$ supports a weak-Markov equilibrium on $[0, \bar{q}]$, then there exists $(P, R)$ which supports a weak-Markov equilibrium on $[0,1]$.

Proof. We extend $\left(R_{\bar{q}}, P_{\bar{q}}\right)$ to some $\left[0, \bar{q}^{\prime}\right]$. Define

$$
R_{\bar{q}^{\prime}}(q)=\max _{0 \leq y \leq \min \{\bar{q}, q\}} \int_{y}^{q} v(z) d\left[n z^{n-1}-(n-1) z^{n}\right]+P_{\bar{q}}(y) n(q-y) y^{n-1}+e^{-r \Delta} R_{\bar{q}}(y)
$$

with $y_{\bar{q}^{\prime}}(q)$ as the supremum of the argmax correspondence. Moreover, we define $P_{\bar{q}^{\prime}}(q)$ by

$$
v(q)-P_{\bar{q}^{\prime}}(q)=e^{-r \Delta}\left[v(q)-\frac{\left(y_{\bar{q}^{\prime}}(q)\right)^{n-1}}{q^{n-1}} P_{\bar{q}}\left(y_{\bar{q}^{\prime}}(q)\right)-\frac{1}{q^{n-1}} \int_{y_{\bar{q}^{\prime}}(q)}^{q} v(x) d x^{n-1}\right] .
$$

For $\bar{q}^{\prime}=\min \left\{1, \sqrt[n]{\bar{q}^{n}+\left(1-e^{-r \Delta}\right) R_{\bar{q}}(\bar{q})}\right\}$, the constraint in the maximization in the definition of $R_{\bar{q}^{\prime}}(q)$ is not binding and moreover

$$
R_{\bar{q}^{\prime}}(q)=\max _{0 \leq y \leq q} \int_{y}^{q} v(z) d\left[n z^{n-1}-(n-1) z^{n}\right]+P_{\bar{q}^{\prime}}(q) n(q-y) y^{n-1}+e^{-r \Delta} R_{\bar{q}^{\prime}}(y)
$$

For $y \in[\bar{q}, q]$ we have

$$
\begin{aligned}
& \int_{y}^{q} v(z) d\left[n z^{n-1}-(n-1) z^{n}\right]+P_{\bar{q}^{\prime}}(q) n(q-y) y^{n-1}+e^{-r \Delta} R_{\bar{q}^{\prime}}(y) \\
\leq & q^{n}-y^{n}+e^{-r \Delta} R_{\bar{q}^{\prime}}(q) \\
\leq & \left(1-e^{-r \Delta}\right) R_{\bar{q}}(\bar{q})+e^{-r \Delta} R_{\bar{q}^{\prime}}(q) \\
\leq & \left(1-e^{-r \Delta}\right) R_{\bar{q}^{\prime}}(q)+e^{-r \Delta} R_{\bar{q}^{\prime}}(q) \\
\leq & R_{\bar{q}^{\prime}}(q) .
\end{aligned}
$$

In the first step, we have used that the payments $v(z)$ and $P_{\bar{q}^{\prime}}(q)$ are less than or equal to one. In the second step, we have used that $\bar{q}^{\prime}=\min \left\{1, \sqrt[n]{\bar{q}^{n}+\left(1-e^{-r \Delta}\right) R_{\bar{q}}(\bar{q})}\right\}$; since $\bar{q} \leq y \leq q \leq \bar{q}^{\prime}$, this implies $q^{n}-y^{n} \leq\left(1-e^{-r \Delta}\right) R_{\bar{q}}(\bar{q})$. The third step uses $R_{\bar{q}}(\bar{q})=R_{\bar{q}^{\prime}}(\bar{q})$ and that $R_{\bar{q}^{\prime}}$ is increasing. Thus $\left(P_{\bar{q}^{\prime}}, R_{\bar{q}^{\prime}}\right)$ supports a weak-Markov equilibrium on $\left[0, \bar{q}^{\prime}\right]$. Since $R_{\bar{q}}(\bar{q})>0$, a finite number of repetitions suffices to extend $\left(P_{\bar{q}}, R_{\bar{q}}\right)$ to the entire interval $[0,1]$.

To complete the proof, AD replace the lower tail distribution on the interval $[0, \bar{q}]$ by a uniform distribution. For the uniform distribution, a weak-Markov equilibrium can be constructed explicitly. In the auction case, this has been shown by McAfee and Vincent (1997). Therefore, Lemma 29 
implies that for the modified distribution with a uniform part at the lower end, a weak-Markov equilibrium exists. The final step is to show that the functions $(P, R)$ that support the equilibrium for the modified distribution converge to functions that support a weak-Markov equilibrium for the original distribution as $\bar{q} \rightarrow 0$.

Proof of Proposition 5. As in AD, we consider a sequence of valuation functions

$$
v_{\eta}(q)= \begin{cases}v(q), & \text { if } q \geq \frac{1}{\eta} \\ v\left(\frac{1}{\eta}\right) \eta q, & \text { otherwise. }\end{cases}
$$

This corresponds to the original distribution except that on the interval $[0,1 / \eta]$, we have made the distribution uniform. McAfee and Vincent (1997) show that there exist $\left(\tilde{P}_{1 / \eta}, \tilde{R}_{1 / \eta}\right)$ that support a weak-Markov equilibrium on $[0,1 / \eta]$. Hence, by Lemma 29 , for each $\eta=1,2, \ldots$, there exists a pair $\left(P_{\eta}, R_{\eta}\right)$ that supports a weak-Markov equilibrium on $[0,1]$. As in $\mathrm{AD}$, we can assume that $P_{\eta}$ converges point-wise for all rationals to some function $\Phi(s), s \in \mathbb{Q} \cap[0,1]$ and taking left limits we can extend this limit to a non-decreasing, left-continuous function $P:[0,1] \rightarrow[0,1]$. Also, by Lemma 28, after taking a sub-sequence, we may assume that $\left(R_{n}\right)$ converges uniformly to a continuous function $R$. We have to show that $(P, R)$ supports a weak-Markov equilibrium for $v$. But given Lemma 28 and 29, only minor modifications are needed to apply the proof of Theorem 4.2 from $\mathrm{AD}$.

\section{B.2 Proof of Proposition 6}

We want to show that the profit of the seller in any weak-Markov equilibrium of a subgame that starts with the posterior $[0, q]$, converges (uniformly over $q$ ) to $\Pi^{E}(q)$ as $\Delta \rightarrow 0$. The proof consists of two main steps. The first step shows that for any type $\xi \in[0,1]$, any $\Delta>0$, and any weak-Markov equilibrium supported by some pair $(P, R)$, the expected payment that the seller can extract from type $\xi$ is bounded by $\xi^{n-1} P(\xi)$. We prove this by showing that the expected payment conditional on winning is bounded by $P(\xi)$.

Lemma 30. Let $(P, R)$ support a weak-Markov equilibrium in the game for $\Delta>0$. Suppose that in this equilibrium, type $\xi \in[0,1]$ trades in period $t$, let the posterior in period $t$ be $q_{t} \geq \xi$, and denote the marginal type in period $t$ by $q_{t}^{+} \leq \xi$. Then we have

$$
P(\xi) \geq \int_{q_{t}^{+}}^{\xi} v(x) \frac{d x^{n-1}}{\xi^{n-1}}+\frac{\left(q_{t}^{+}\right)^{n-1}}{\xi^{n-1}} P\left(q_{t}^{+}\right), \quad \forall \xi \in[0,1],
$$

and hence

$$
R(q) \leq \int_{0}^{q} P(x) d x^{n}, \quad \forall q \in(0,1] .
$$


Proof. For $q_{t}^{+}=\xi$ the RHS of the first inequality becomes $P\left(q_{t}^{+}\right)=P(\xi)$. Hence it suffices to show that

$$
\int_{q}^{\xi} v(x) d x^{n-1}+q^{n-1} P(q)
$$

is increasing in $q$. For $q>\hat{q}$ we have

$$
\begin{aligned}
& \int_{q}^{\xi} v(x) d x^{n-1}+q^{n-1} P(q)-\int_{\hat{q}}^{\xi} v(x) d x^{n-1}-\hat{q}^{n-1} P(\hat{q}) \\
= & q^{n-1} P(q)-\hat{q}^{n-1} P(\hat{q})-\int_{\hat{q}}^{q} v(x) d x^{n-1}
\end{aligned}
$$

Using (B.2), we have

$$
\begin{aligned}
& q^{n-1} P(q)-\hat{q}^{n-1} P(\hat{q}) \\
= & \left(1-e^{-r \Delta}\right) q^{n-1} v(q)+e^{-r \Delta} \int_{y(q)}^{q} v(x) d x^{n-1}+e^{-r \Delta}(y(q))^{n-1} P(y(q)) \\
& -\left(1-e^{-r \Delta}\right) \hat{q}^{n-1} v(\hat{q})-e^{-r \Delta} \int_{y(\hat{q})}^{\hat{q}} v(x) d x^{n-1}-e^{-r \Delta}(y(\hat{q}))^{n-1} P(y(\hat{q})) \\
= & \left(1-e^{-r \Delta}\right)\left(q^{n-1} v(q)-\hat{q}^{n-1} v(\hat{q})\right)+e^{-r \Delta}\left((y(q))^{n-1} P(y(q))-(y(\hat{q}))^{n-1} P(y(\hat{q}))\right) \\
& +e^{-r \Delta} \int_{\hat{q}}^{q} v(x) d x^{n-1}-e^{-r \Delta} \int_{y(\hat{q})}^{y(q)} v(x) d x^{n-1}
\end{aligned}
$$

and hence

$$
\begin{aligned}
& q^{n-1} P(q)-\hat{q}^{n-1} P(\hat{q})-\int_{\hat{q}}^{q} v(x) d x^{n-1} \\
= & \left(1-e^{-r \Delta}\right)\left(q^{n-1} v(q)-\hat{q}^{n-1} v(\hat{q})\right)+e^{-r \Delta}\left((y(q))^{n-1} P(y(q))-(y(\hat{q}))^{n-1} P(y(\hat{q}))\right) \\
& -\left(1-e^{-r \Delta}\right) \int_{\hat{q}}^{q} v(x) d x^{n-1}-e^{-r \Delta} \int_{y(\hat{q})}^{y(q)} v(x) d x^{n-1} \\
= & e^{-r \Delta}\left((y(q))^{n-1} P(y(q))-(y(\hat{q}))^{n-1} P(y(\hat{q}))-\int_{y(\hat{q})}^{y(q)} v(x) d x^{n-1}\right) \\
& +\left(1-e^{-r \Delta}\right) \int_{\hat{q}}^{q} v^{\prime}(x) x^{n-1} d x
\end{aligned}
$$

Proceeding inductively, we get

$$
q^{n-1} P(q)-\hat{q}^{n-1} P(\hat{q})-\int_{\hat{q}}^{q} v(x) d x^{n-1}=\sum_{k=0}^{\infty} e^{-k \Delta}\left(1-e^{-r \Delta}\right) \int_{y^{k}(\hat{q})}^{y^{k}(q)} v^{\prime}(x) x^{n-1} d x>0,
$$

where $y^{k}(\cdot)$ denotes the function obtained by applying $y(\cdot) k$ times. This shows the first inequality.

For the second inequality, notice that the first inequality implies that the seller's profit if the 
posterior is $q$, satisfies

$$
R(q) \leq \int_{0}^{q} e^{-r(T(x)-t)} P(x) d x^{n-1},
$$

where $T(x)$ denotes the trading time of type $x$ in the weak-Markov equilibrium. This implies the second inequality.

For the second step, fix the distribution and the corresponding function $v$ and define $v_{x}:[0,1] \rightarrow$ $[0,1]$ for all $x \in(0,1]$.

$$
v_{x}(q):=\frac{v(q x)}{v(x)} .
$$

Using Helly's selection theorem, we can extend this definition to $x=0$, by taking the a.e.-limit of a subsequence of functions $v_{x}$. Denote by $\mathcal{E}^{w M}(\Delta, x)$ the weak-Markov equilibria of the game with discount factor $\Delta$ and distribution given by $v_{x}$ where $x \rightarrow 0$. Slightly abusing notation we write $(P, R) \in \mathcal{E}^{w M}(\Delta, x)$ for a weak-Markov equilibrium that is supported by functions $(P, R)$. We show that there is an upper bound for $P(1)$ that converges to the expected payment in a second price auction without reserve price as $\Delta \rightarrow 0$, and the convergence is uniform over $x$.

Lemma 31. Fix $v(\cdot)$. For all $\varepsilon>0$, there exists $\Delta_{\varepsilon}>0$ such that for all $\Delta \leq \Delta_{\varepsilon}$, all $x \in[0,1]$, and all $(P, R) \in \mathcal{E}^{w M}(\Delta, x)$,

$$
P(1) \leq \int_{0}^{1} v_{x}(s) d s^{n-1}+\varepsilon .
$$

Proof. Suppose not. Then there exist sequences $\Delta_{m} \rightarrow 0$ and $x_{m} \rightarrow \bar{x}$ such that for all $m \in \mathbb{N}$, there exist equilibria $\left(P_{m}, R_{m}\right) \in \mathcal{E}^{w M}\left(\Delta_{m}, x_{m}\right)$ such that for all $m$,

$$
P_{m}(1)>\int_{0}^{1} v_{x_{m}}(s) d s^{n-1}+\varepsilon
$$

By a similar argument as in the proof of Theorem 4.2 of $\mathrm{AD}$, we can construct a limiting pair $(\bar{P}, \bar{R})$, where $\bar{P}$ is left-continuous and non-decreasing, $P_{m}$ converges point-wise to $\bar{P}$ for all rationals, and $R_{m}$ converges uniformly to $\bar{R}$. Obviously, we have

$$
\bar{P}(1) \geq \int_{0}^{1} v_{\bar{x}}(s) d s^{n-1}+\varepsilon
$$

Left-continuity implies that there exists $\bar{q}<1$ such that

$$
\bar{P}(\bar{q}) \geq \int_{0}^{1} v_{\bar{x}}(s) d s^{n-1}+\frac{\varepsilon}{2}
$$

Using an argument from the proof of Theorem 5.4 in $\mathrm{AD}$, we can show that

$$
\bar{R}(1) \geq \int_{\bar{q}}^{1} \bar{P}(s) d s^{n}+\Pi^{E}(\bar{q}) \geq \Pi^{E}(1)+(1-\bar{q}) \frac{\varepsilon}{2},
$$


where we have used (B.4) to show the second inequality. Hence, we have

$$
R_{m}(1) \rightarrow \bar{R}(1) \geq \Pi^{E}(1)+(1-\bar{q}) \frac{\varepsilon}{2}
$$

But this implies that there must exist a type $\hat{q}>0$, a time $t>0$, and $\bar{m}$ such that for all $m>\bar{m}$,

$$
T_{m}(\hat{q}) \geq t
$$

where $T_{m}(\cdot)$ is the trading time function in the weak-Markov equilibrium supported by $\left(P_{m}, R_{m}\right)$. To see this, note that delay for low types is needed to increase the seller's revenue beyond the revenue from an efficient auction.

With this observation, we can conclude the proof using a similar argument as in Case I of the proof of Theorem 5.4 in AD. From Lemma 30 we know that the maximal expected payment conditional on winning that a buyer of type $q$ has to make in equilibrium is given by $P_{m}(q)$. This implies that

$$
R_{m}(1) \leq \int_{\hat{q}}^{1} P_{m}(z) d z^{n}+e^{-r t} R_{m}(\hat{q})
$$

In the limit we have

$$
\bar{R}(1) \leq \int_{\hat{q}}^{1} \bar{P}(z) d z^{n}+e^{-r t} \bar{R}(\hat{q}) .
$$

On the other hand, the same argument that we used to obtain (B.5) yields

$$
\bar{R}(1) \geq \int_{0}^{1} \bar{P}(z) d z^{n}
$$

Combining (B.6) and (B.7) we get

$$
\int_{0}^{\hat{q}} \bar{P}(z) d z^{n} \leq e^{-r t} \bar{R}(\hat{q}),
$$

which implies

$$
\bar{R}(\hat{q})>\int_{0}^{\hat{q}} \bar{P}(z) d z^{n}
$$

since $t>0$. But Lemma 30 implies the opposite inequality which is a contradiction.

Using this Lemma, we can show that for a given $v(\cdot)$, the difference between the continuation profit at $[0, q]$ and $\Pi^{E}(q)$, divided by $v(q)$ converges uniformly to zero.

Lemma 32. Fix $v(\cdot)$. For all $\varepsilon>0$, there exists $\Delta_{\varepsilon}>0$ such that for all $\Delta \leq \Delta_{\varepsilon}$, all $x \in[0,1]$, and all $(P, R) \in \mathcal{E}^{w M}(\Delta, 1)$,

$$
\frac{R(x)}{x^{n}}-\Pi^{E}(v(x)) \leq \varepsilon v(x) .
$$


Proof. The statement of the Lemma is equivalent to the statement that for all $\varepsilon>0$, there exists $\Delta_{\varepsilon}>0$ such that for all $\Delta \leq \Delta_{\varepsilon}$, all $x \in[0,1]$, and all $(P, R) \in \mathcal{E}^{w M}(\Delta, x)$,

$$
R\left(1 \mid v_{x}\right)-\Pi^{E}\left(1 \mid v_{x}\right) \leq \varepsilon
$$

This equivalence holds because truncating and rescaling the function $v(\cdot)$ leads to the following transformations:

$$
\begin{gathered}
\frac{R(x \mid v)}{x^{n}}=v(x) R\left(1 \mid v_{x}\right), \\
\Pi^{E}(v(x))=v(x) \Pi^{E}\left(1 \mid v_{x}\right) .
\end{gathered}
$$

To show (B.8), we combine Lemmas 30 and 31, and use that $P\left(z \mid v_{x}\right)=v_{x}(z) P\left(1 \mid v_{z \cdot x}\right)$ to get for all $x \in[0,1]$,

$$
\begin{aligned}
R(1) & \leq \int_{0}^{1} P\left(z \mid v_{x}\right) d z^{n} \\
& =\int_{0}^{1} v_{x}(z) P\left(1 \mid v_{x \cdot z}\right) d z^{n} \\
& \leq \int_{0}^{1} v_{x}(z)\left(\int_{0}^{1} v_{x \cdot z}(s) d s^{n-1}+\varepsilon\right) d z^{n} \\
& =\int_{0}^{1}\left(\int_{0}^{1} v_{x}(s z) d s^{n-1}\right) d z^{n}+\varepsilon \int_{0}^{1} v_{x}(z) d z^{n} \\
& \leq \int_{0}^{1}\left(\int_{0}^{z} v_{x}(s) \frac{d s^{n-1}}{z^{n-1}}\right) d z^{n}+\varepsilon \\
& =\Pi^{E}\left(1 \mid v_{x}\right)+\varepsilon
\end{aligned}
$$

This allows us to complete the proof of Proposition 6 .

Proof of Proposition 6. Translated into the notation of the main paper, Lemma 32 implies that for a given distribution function $F$, for all $\tilde{\varepsilon}>0$, there exists $\Delta_{\tilde{\varepsilon}}>0$ such that for all $\Delta \leq \Delta_{\tilde{\varepsilon}}$, all $v \in[0,1]$, and all weak-Markov equilibria $(p, b) \in \mathcal{E}^{w M}(\Delta)$, we have

$$
\Pi^{\Delta}(p, b \mid v) \leq \Pi^{E}(v)+\tilde{\varepsilon} v .
$$

As in the proof of Lemma 9, we can show that under Assumption A3, there exits a constant $B>0$ such that $\Pi^{E}(v) \geq B v$ for all $v \in[0,1]$. If we chose $\tilde{\varepsilon}$ sufficiently small we have

$$
\tilde{\varepsilon} \leq B \varepsilon
$$




$$
\begin{aligned}
\Longleftrightarrow \quad \tilde{\varepsilon} v & \leq B \varepsilon v, \\
\Longleftrightarrow \quad \tilde{\varepsilon} v & \leq \varepsilon \Pi^{E}(v), \\
\Longleftrightarrow \quad \Pi^{E}(v)+\tilde{\varepsilon} v & \leq(1+\varepsilon) \Pi^{E}(v) .
\end{aligned}
$$

This implies that

$$
\Pi^{\Delta}(p, b \mid v) \leq(1+\varepsilon) \Pi^{E}(v)
$$

for all $\Delta \leq \Delta_{\varepsilon}:=\Delta_{\tilde{\varepsilon}}$ for $\tilde{\varepsilon}$ sufficiently small. 